Flight Testing Small UAVs for Aerodynamic Parameter Estimation

\author{
A Thesis \\ presented to \\ the Faculty of California Polytechnic State University \\ San Luis Obispo
}

In Partial Fulfillment

of the Requirements for the Degree

Master of Science in Aerospace Engineering

by

Adam T. Chase

February 2014 


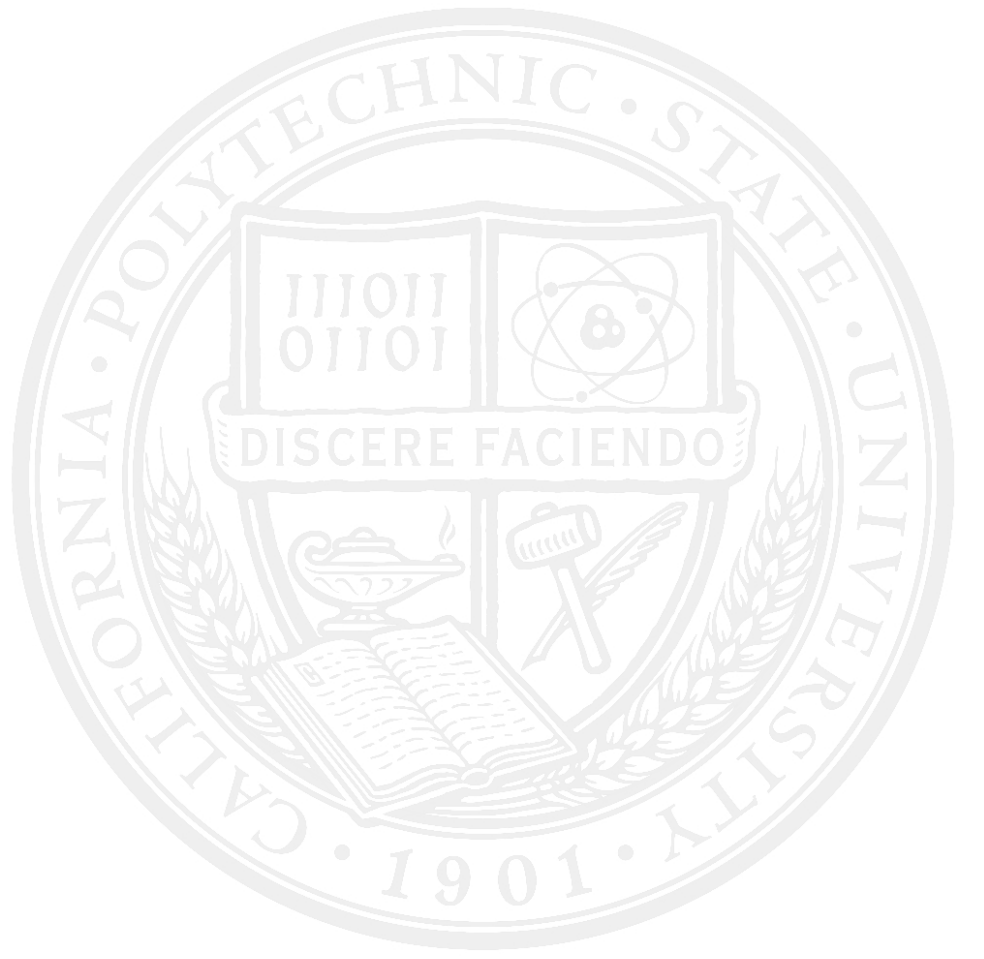

(c) 2014

Adam T. Chase

ALL RIGHTS RESERVED 


\section{COMMITTEE MEMBERSHIP}

TITLE:

AUTHOR:

DATE SUBMITTED:

COMMITTEE CHAIR: Robert McDonald, Ph.D.

Associate Professsor, Aerospace Engineering

COMMITTEE MEMBER: Eric Mehiel, Ph.D.

Associate Professor, Aerospace Engineering

COMMITTEE MEMBER: Russell Westphal, Ph.D.

Professor, Mechanical Engineering

COMMITTEE MEMBER: Kurt Colvin, Ph.D.

Professor, Industrial and Manufacturing Engineering 


\section{ABSTRACT \\ Flight Testing Small UAVs for Aerodynamic Parameter Estimation}

Adam T. Chase

A flight data acquisition system was developed to aid unmanned vehicle designers in verifying the vehicle's design performance. The system is reconfigurable and allows the designer to choose the correct combination of complexity, risk, and cost for a given flight test. The designer can also reconfigure the system to meet packaging and integration requirements. System functionality, repeatbility, and accuracy was validated by collecting data during multiple flights of a radio-controlled aircraft. Future work includes sensor fusion, thrust prediction methods, stability and control derivative estimation, and growing Cal Poly's small-scale component aerodynamic database. 


\section{ACKNOWLEDGMENTS}

I'd first like to thank my friends and fellow Flight Lab people. Each of you brought something good to my time at Poly. $\mathrm{B}^{2}$ and Cory, you guys were awesome roommates and made the Stafford house fun. Alex, Brian, Christian, Trevor, Mike, and the rest of the DBF/Flight Lab crew, you guys made that window-less lab bearable, and occasionally downright enjoyable. \#W9W

I'd also like to thank my committee. Throughout my Cal Poly career every member of the committee has helped me immensely. Dr. Colvin, hosting Akaflieg barbecues at your hangar introduced my to a side of aviation I hadn't seen, and gave me passion for aircraft and flight. Dr. Mehiel, you've provided countless guidance throughout school, ranging from classes and scheduling to Lyapunov stability and Kalman filters. Dr. Westphal, the Edward's project was a great experience for me, and you've been incredibly supportive with anything and everything I've needed help with since. And Dr. McDonald, you've given me countless opportunities, including DBF, Edwards, LENR, Senior Design, R/MAX, this thesis, ... For five and a half years, you've guided me through challenging problems, taught me to think like an engineer, and ignored DBF t-shirts and team names. Thank you, it's been a pleasure.

Finally, my family. The entire family (aunts, uncles, grandparents, etc.) have supported me the whole way through, and I'm very grateful for that. I can't put into words the opportunities and support my parents and sister have given me. Mom, Dad, Jackie: Thank you. You guys are awesome. 


\section{TABLE OF CONTENTS}

LIST OF TABLES Ix

LIST OF FIGURES $\quad$ x

$\begin{array}{lll}\text { Nomenclature } & \text { XV }\end{array}$

1 Introduction $\quad 1$

2 Method $\quad 3$

2.1 Reference Frames . . . . . . . . . . . . . . . . . . . . . 3

2.1.1 North-East-Down (NED) Axes $\left(x_{n e d}, y_{n e d}, z_{n e d}\right) \ldots \ldots$

2.1.2 Body Axes $\left(x_{b}, y_{b}, z_{b}\right) \ldots \ldots \ldots \ldots$

2.1.3 Stability Axes $\left(x_{s}, y_{s}, z_{s}\right) \ldots \ldots \ldots \ldots$

2.1.4 Wind Axes $\left(x_{w}, y_{w}, z_{w}\right) \ldots \ldots \ldots$. . . . . . . . . . 6

2.2 Equations of Motion . . . . . . . . . . . . . . . 6

2.3 Kalman Filter Usage . . . . . . . . . . . . . . . . . . . 8

2.3.1 Linear Kalman Filter . . . . . . . . . . . . . . . . . . . . . . 8

2.3.2 Extended Kalman Filter . . . . . . . . . . . . . . . . . . . . . 10

3 Drag Meta-Modeling 12

3.1 Regression Model - Least Squares Fit . . . . . . . . . . . . . . . . 15

3.2 Regression Model - Kalman Filter . . . . . . . . . . . . . . . . . 16

4 Error Analysis $\quad 18$

4.1 Random Error . . . . . . . . . . . . . . . . . . . . 18

4.2 Least Squares Regression Error . . . . . . . . . . . . . . . . . . . . . . . . . 20

4.3 Kalman Filter Regression Error . . . . . . . . . . . . . . . . . . . . 21

5 Simulation $\quad 23$

5.1 Simulation Environment . . . . . . . . . . . . . . . . 23

5.2 Simulation Inputs . . . . . . . . . . . . . . . . . . . . . 23

5.3 Simulation Results . . . . . . . . . . . . . . . . . . 24 
6 Hardware $\quad 28$

6.1 Flight Computer . . . . . . . . . . . . . . . . . . . . . . . . 28

6.2 Accelerometer . . . . . . . . . . . . . . . . . . . . . . . 29

6.3 Vehicle Mass . . . . . . . . . . . . . . . . . . . . . . . . . . . . 31

6.4 Magnetometers . . . . . . . . . . . . . . . . . . . . 31

6.5 Gyroscope . . . . . . . . . . . . . . . . . . . . . . . 34

6.6 Air Data System . . . . . . . . . . . . . . . . . . . . . . . 35

6.7 GPS Receiver . . . . . . . . . . . . . . . . . . . . . . . 37

6.8 Data Acquisition System Integration _ . . . . . . . . . . . . 38

$\begin{array}{lll}7 & \text { Results } & 39\end{array}$

$7.1 \quad$ Drag Polar . . . . . . . . . . . . . . . . . . . . . . . . . . . . . . . . . . . 39

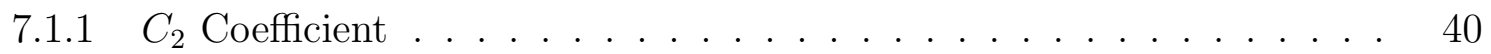

$7.1 .2 \quad$ Drag Break . . . . . . . . . . . . . . . . . . . . . 41

7.1.3 Error Estimation . . . . . . . . . . . . . . . . . . . . . 41

$7.2 \quad$ Lift Curve . . . . . . . . . . . . . . . . . . . . . . . . . . 42

$7.2 .1 \quad C_{L_{M A X}}$ and Stall $\ldots \ldots \ldots \ldots \ldots \ldots \ldots$

7.2 .2 Lift Curve Slope . . . . . . . . . . . . . . . . . . . . . . 44

7.2 .3 Zero Lift Angle of Attack . . . . . . . . . . . . . . . . . 45

7.3 System Repeatability . . . . . . . . . . . . . . . . . . . . . . 45

7.4 System Accuracy . . . . . . . . . . . . . . . . . . . . . . . . 52

$\begin{array}{lll}8 & \text { Summary } & 58\end{array}$

$\begin{array}{lr}\text { Bibliography } & 59\end{array}$

$\begin{array}{ll}\text { A Data Acquisition System Usage } & 62\end{array}$

A.1 Integration . . . . . . . . . . . . . . . . . . . . . . . 62

A.2 Pre-flight Procedure . . . . . . . . . . . . . . . 65

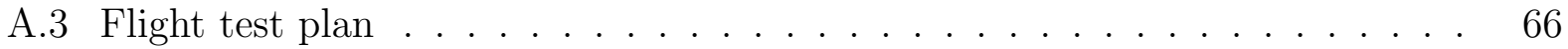

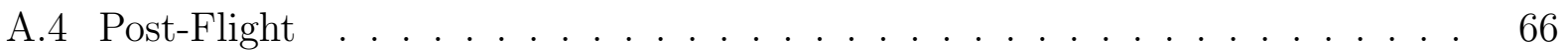

A.5 Embedded Software Protocol . . . . . . . . . . . . . . . . . . . 67

$\begin{array}{lc}\text { B Lessons Learned } & 69\end{array}$

$\begin{array}{ll}\text { C Wiring Schematics } & 72\end{array}$ 
$\begin{array}{ll}\text { D Flight Test Procedure } & 75\end{array}$

D.1 Pre-Flight Preparation . . . . . . . . . . . . . . . . . . . 75

D.1.1 Day Before Test . . . . . . . . . . . . . . . . . 75

D.1.2 Day Of Test ........................ 76

D.2 Flying Field Procedure . . . . . . . . . . . . . . . . . . . 77

D.3 Post-Flight . . . . . . . . . . . . . . . . . . 78

$\begin{array}{lll}\text { E Sample System Ouput } & 79\end{array}$

E.1 Sample System Ouput - Raw Data . . . . . . . . . . . . . . . . 79

E.2 Sample System Ouput - Units Data . . . . . . . . . . . . . . . . . . . 94

E.3 Sample System Ouput - State Data . . . . . . . . . . . . . . . . . . . 106 


\section{LIST OF TABLES}

5.1 Nonlinear Model Results . . . . . . . . . . . . . . . . . . . . . . . . . 25

5.2 Monte Carlo Results . . . . . . . . . . . . . . . . . . . . . . . . . . 27

7.1 Clean Drag Polar Repeatability Testing : $C_{D_{0}}$ Regression Coefficient . . . . 47

7.2 Clean Drag Polar Repeatability Testing : $C_{1}$ Regression Coefficient . . . . 47

7.3 Clean Drag Polar Repeatability Testing : $C_{2}$ Regression Coefficient . . . . 47

7.4 Lift Curve Model . . . . . . . . . . . . . . . . . . . . . . . . . 50

7.5 Ellipticity Value with $C_{1}$ From Flight and XFOIL . . . . . . . . . 51

7.6 Lift Curve Slope with $C_{1}$ From Flight and XFOIL . . . . . . . . . . . 52

7.7 Dirty Drag Polar Repeatability Testing : $C_{D_{0}}$ Regression Coefficient . . . . 55

7.8 Dirty Drag Polar Repeatability Testing : $C_{1}$ Regression Coefficient . . . . . 55

7.9 Dirty Drag Polar Repeatability Testing : $C_{2}$ Regression Coefficient . . . . . 55

A.1 Air Data System Setup . . . . . . . . . . . . . . . . . . . . . . 62

A.2 Air Data System Setup . . . . . . . . . . . . . . . . . . . 63

A.3 Available Commands for mainScript.ino . . . . . . . . . . . 68

A.4 Available Commands for calibration.ino . . . . . . . . . . . . . 68 


\section{LIST OF FIGURES}

2.1 NED Frame of Reference ${ }^{[1]} \ldots \ldots \ldots \ldots$. . . . . . . . . . . . 4

2.2 Body Axes Definition ${ }^{[2]} \ldots \ldots \ldots \ldots \ldots$

2.3 Stability Axes Definition . . . . . . . . . . . . . . . 5

2.4 Wind Axes Definition . . . . . . . . . . . . . . . . . . . . 6

3.1 Drag Contribution Types . . . . . . . . . . . . . . . . 12

3.2 NACA 4412 Lift Curve . . . . . . . . . . . . . . . . . . . . . . 13

3.3 NACA 4412 Drag Polar . . . . . . . . . . . . . . . . . 13

3.4 NACA $4412 K_{1}$ Estimation . . . . . . . . . . . . . . . . . 13

3.5 Downwash Caused By Wingtip Vortices ${ }^{[3]} \ldots \ldots$. . . . . . . . . . . . 14

3.6 Induced Drag Free Body Diagram . . . . . . . . . . . . . . . . . . 14

4.1 Heteroskedastic Error from Simulated Flight . . . . . . . . . . . . . . . 20

5.1 Data Analysis Verification (No Noise) . . . . . . . . . . . . . . 24

5.2 Drag Polar Prediction of Simulated Test Flight _ . . . . . . . . . . . 25

$5.3 C_{D_{0}}$ Monte Carlo Simulation . . . . . . . . . . . . . . . 26

$5.4 C_{1}$ Monte Carlo Simulation . . . . . . . . . . . . . 26

$5.5 C_{2}$ Monte Carlo Simulation . . . . . . . . . . . . . . 27

6.1 Arduino Due Flight Computer . . . . . . . . . . . . . . . . 28

6.2 Logic Level Converter Circuit _ . . . . . . . . . . . . . . . . . . . . . . . . . 29

6.3 ADXL-362 Schematic . . . . . . . . . . . . . . . . . . 29

6.4 Calibration Results of ADXL-362 Accelerometer . . . . . . . . . . . . . . 30

6.5 Honeywell HMR-2300 3-D Magnetometer . . . . . . . . . . . . . . . . . . 31

6.6 HMR-2300 Logic Level Circuit . . . . . . . . . . . . . . . . . . . . . . 32

6.7 HMC-5883L Schematic . . . . . . . . . . . . . . . . . . 33

6.8 Soft- and Hard-iron Calibration for HMC-5883L . . . . . . . . . . . . . . . 33

6.9 ITG-3200 Eagle Schematic . . . . . . . . . . . . . . . . . . . . . . 34 
6.10 Five-Hole Probe Adapter . . . . . . . . . . . . . . . . . . . . . . . . . 35

6.11 All Sensors 5-INCH-D-DO Pressure Sensor . . . . . . . . . . . . . . . 36

6.12 Dallas Semiconductors' DS18B20 Digital Temperature Sensors . . . . . . . . 37

6.13 CGS Shop Board for uBlox LEA-6T . . . . . . . . . . . . . . . . 37

7.1 System Integration into 0.60-size R/C Piper Cub $\ldots \ldots \ldots$

7.2 Drag Polar from Flight Test ～. . . . . . . . . . . . . . . . . . . 40

7.3 Drag Polar Residuals from Flight Test . . . . . . . . . . . . . . . . . . 42

7.4 Lift Curve from Flight Test ．. . . . . . . . . . . . . . . . . . 43

7.5 Angle of Attack History from Flight Test . . . . . . . . . . . . . . . 44

7.6 Drag Polar Repeatability Testing _ . . . . . . . . . . . . . . . . . 46

7.7 Correlation of Regression Model and Flight Data . . . . . . . . . . . . . . 48

7.8 Residuals of Regression Model and Flight Data . . . . . . . . . . . . . 49

7.9 Lift Curve Repeatability Testing . . . . . . . . . . . . . . . . . . . 50

7.10 XFOIL-based $C_{1}$ Estimation $\ldots \ldots \ldots \ldots \ldots \ldots \ldots$

7.11 Parasite Drag Cone Integration _ . . . . . . . . . . . . . . 53

7.12 Cone Drag as a Function of Half Angle ${ }^{[4]} \ldots \ldots \ldots \ldots . \ldots . \ldots 53$

7.13 Clean vs. Dirty Drag Polar . . . . . . . . . . . . . . . . . . 56

7.14 Clean vs. Dirty Lift Curve . . . . . . . . . . . . . . . . . . . . 57

A.1 Port Description for Pressure Sensors _ . . . . . . . . . . . . . . . . 64

C.1 Arduino Due Flight Data Recorder v3.20BOB Schematic . . . . . . . . . 72

C.2 Arduino Due Flight Data Recorder v3.20BOB Layout . . . . . . . . . . . 73

C.3 Pressure Board v2.20 Schematic . . . . . . . . . . . . . . . . . . . . . 73

C.4 Pressure Board v2.20 Layout . . . . . . . . . . . . . . . . . . . 74

E.1 accelX vs. Time . . . . . . . . . . . . . . . . . . . . . . 79

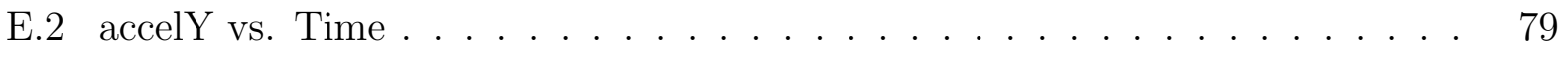

E.3 accelZ vs. Time . . . . . . . . . . . . . . . . . . . . . . . . 80

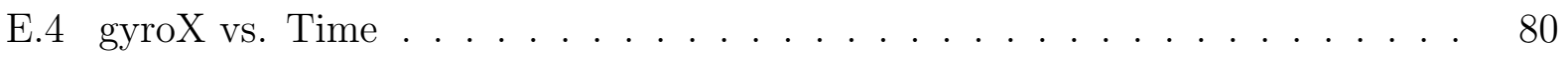

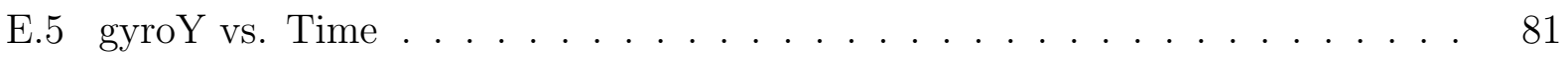

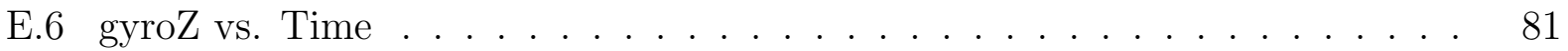

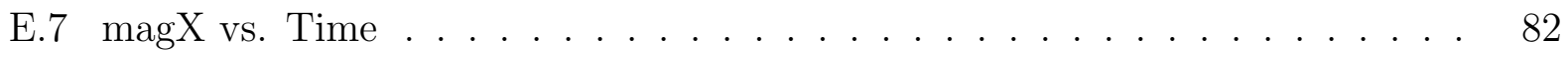


E.8 magY vs. Time . . . . . . . . . . . . . . . . . . . . 83

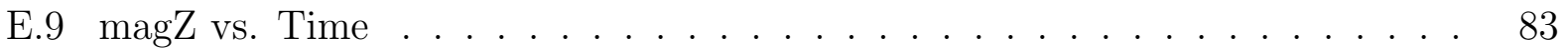

E.10 hmcX vs. Time . . . . . . . . . . . . . . . . . . . . . 84

E.11 hmcZ vs. Time . . . . . . . . . . . . . . . . . . . . . . . . . 84

E.12 hmcY vs. Time . . . . . . . . . . . . . . . . . 85

E.13 press0 vs. Time . . . . . . . . . . . . . . . . . . 85

E.14 press1 vs. Time . . . . . . . . . . . . . . . . . . . . 85

E.15 press 2 vs. Time . . . . . . . . . . . . . . . . 86

E.16 press 3 vs. Time . . . . . . . . . . . . . . . . . . . 86

E.17 gpsLat vs. Time . . . . . . . . . . . . . . . . . . . 87

E.18 gpsLong vs. Time . . . . . . . . . . . . . . . . . . . 87

E.19 gpsSpd vs. Time . . . . . . . . . . . . . . . . . . . 88

E.20 gpsCrs vs. Time . . . . . . . . . . . . . . . . . . . . . . 88

E.21 date vs. Time . . . . . . . . . . . . . . . . . . . . . . . . . . . . . . 89

E.22 CS vs. Time . . . . . . . . . . . . . . . . . . . . . . . . . 89

E.23 temperature vs. Time . . . . . . . . . . . . . . . . . . . . . 90

E.24 pwm0 vs. Time . . . . . . . . . . . . . . . . . . . . . 90

E.25 pwm1 vs. Time . . . . . . . . . . . . . . . . . . . . . 91

E.26 pwm2 vs. Time . . . . . . . . . . . . . . . . . . . . . . . 91

E.27 pwm3 vs. Time . . . . . . . . . . . . . . . . . . . . . . . 92

E.28 pwm4 vs. Time . . . . . . . . . . . . . . . . . . . . 92

E.29 pwm5 vs. Time . . . . . . . . . . . . . . . . . . . . . . . 92

E.30 pwm6 vs. Time . . . . . . . . . . . . . . . . . . . 93

E.31 pwm7 vs. Time . . . . . . . . . . . . . . . . . . . . . . 93

E.32 deltaT vs. Time . . . . . . . . . . . . . . . . . . . . . . . . 94

E.33 accelX vs. Time . . . . . . . . . . . . . . . . . . . . . . 94

E.34 accelY vs. Time . . . . . . . . . . . . . . . . . . . . . . 95

E.35 accelZ vs. Time . . . . . . . . . . . . . . . . . . . . . 95

E.36 gyroX vs. Time . . . . . . . . . . . . . . . . . 95

E.37 gyroY vs. Time . . . . . . . . . . . . . . . . . . 96

E.38 gyroZ vs. Time . . . . . . . . . . . . . . . . . . 96

E.39 magX vs. Time . . . . . . . . . . . . . . . . . 96 
E.40 magY vs. Time . . . . . . . . . . . . . . . . . . . . 97

E.41 magZ vs. Time . . . . . . . . . . . . . . . . . . . . . . . . . . 97

E.42 hmcX vs. Time . . . . . . . . . . . . . . . . . . . . . . . 97

E.43 hmcZ vs. Time . . . . . . . . . . . . . . . . . . . . . . . . . . . 98

E.44 hmcY vs. Time . . . . . . . . . . . . . . . . . . . . . 98

E.45 press 0 vs. Time . . . . . . . . . . . . . . . . . . . . 98

E.46 press1 vs. Time . . . . . . . . . . . . . . . . . . . . . . . . . . . 99

E.47 press2 vs. Time . . . . . . . . . . . . . . . . . . . . . . . . . . . 99

E.48 press 3 vs. Time . . . . . . . . . . . . . . . . . . . . . . . . . . . . . . . 99

E.49 gpsLat vs. Time . . . . . . . . . . . . . . . . . . . 100

E.50 gpsLong vs. Time . . . . . . . . . . . . . . . . . . . 100

E.51 gpsSpd vs. Time . . . . . . . . . . . . . . . . . . . . 100

E.52 gpsCrs vs. Time . . . . . . . . . . . . . . . . . . . . . . 101

E.53 date vs. Time . . . . . . . . . . . . . . . . . . . . . . . . . 101

E.54 CS vs. Time . . . . . . . . . . . . . . . . . . . . . . 102

E.55 temperature vs. Time . . . . . . . . . . . . . . . . . . . . . 102

E.56 pwm0 vs. Time . . . . . . . . . . . . . . . . . . . . . 102

E.57 pwm1 vs. Time . . . . . . . . . . . . . . . . . . . 103

E.58 pwm2 vs. Time . . . . . . . . . . . . . . . . . . . . . 103

E.59 pwm3 vs. Time . . . . . . . . . . . . . . . . . . . . . 103

E.60 pwm4 vs. Time . . . . . . . . . . . . . . . . . . . . . . 104

E.61 pwm5 vs. Time . . . . . . . . . . . . . . . . . . . . . 104

E.62 pwm6 vs. Time . . . . . . . . . . . . . . . . . . . . . . . . . . . . 104

E.63 pwm7 vs. Time . . . . . . . . . . . . . . . . . . . . 105

E.64 deltaT vs. Time . . . . . . . . . . . . . . . . . . . . . 105

E.65 qbar vs. Time . . . . . . . . . . . . . . . . . . . . . 106

E.66 rho vs. Time . . . . . . . . . . . . . . . . . . . 106

E.67 vinf vs. Time . . . . . . . . . . . . . . . . . . . 107

E.68 alphaP vs. Time . . . . . . . . . . . . . . . . . . . 107

E.69 betaP vs. Time . . . . . . . . . . . . . . . . . . . . . . 107

E.70 rollRate vs. Time . . . . . . . . . . . . . . . . . . . . . . . . . . . 108

E.71 pitchRate vs. Time . . . . . . . . . . . . . . . . . . . . . . . . 108 
E.72 yawRate vs. Time . . . . . . . . . . . . . . . . . . . . . . . 108

E.73 accelX vs. Time . . . . . . . . . . . . . . . . . . . . . . . . . . . . 109

E.74 accelY vs. Time . . . . . . . . . . . . . . . . . . . . . . . . . . 109

E.75 accelZ vs. Time . . . . . . . . . . . . . . . . . . . . . . . . 109

E.76 CD vs. Time . . . . . . . . . . . . . . . . . . . 110

E.77 CY vs. Time . . . . . . . . . . . . . . . . . . . 110

E.78 CL vs. Time . . . . . . . . . . . . . . . . . . . . 110

E.79 D vs. Time . . . . . . . . . . . . . . . . . . . 111

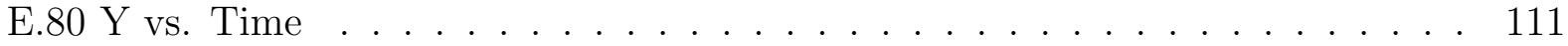

E.81 L vs. Time . . . . . . . . . . . . . . . . . . . . . . . 111 


\title{
Nomenclature
}

\author{
Acronyms \\ $\bar{R}_{n}^{m} \quad$ Rotation matrix from $\mathrm{m}$ to $\mathrm{n}$ \\ $\sigma \quad$ Standard deviation \\ $\vec{F}_{A} \quad$ Aerodynamic forces \\ $\vec{F}_{G} \quad$ Gravitational forces \\ AR Aspect ratio \\ $C_{2} \quad$ Drag polar coefficient for $C_{L}^{2}$ terms \\ $C_{D} \quad$ Drag coefficient \\ $C_{L} \quad$ Lift coefficient \\ $C_{D_{0}} \quad$ Parasite drag coefficient \\ $P_{S} \quad$ Static pressure \\ BEC Battery elimination circuit \\ CFD Computational fluid dynamics \\ CG Center of gravity \\ DOF Degree of freedom \\ e Aircraft Lift Distribution's Ellipticity \\ e Wing lift distribution \\ EFR Education Flight Range \\ EKF Extended Kalman filter \\ ESC Electronic speed controller \\ FSO Full scale output \\ G Gravity \\ GUI Graphical user interface \\ $\mathrm{I}^{2} \mathrm{C} \quad$ Inter-integrated circuit \\ I/O Input-output \\ IC Integrated circuit \\ INS Inertial navigation system \\ LCC Leadless chip carrier \\ LGA Land grid array \\ LiPo Lithium polymer \\ NED North east down frame \\ OLS Ordinary least squares \\ PCB Printed circuit board \\ PWM Pulse width modulation
}


QFN Quad-flats no-leads

R/C Radio-controlled

RMS Root mean squared

RPM Rotations per minute
SPI Serial peripheral interface

UAS Unmanned aerial system

$\alpha \quad$ Angle of attack 


\subsection{Introduction}

Aircraft designers often use model fits and "rules of thumb" to successfully complete

designs, with many of these guidelines available in various design textbooks. [5],[6],[7] These models and practices have been established based on years of data analysis and validation that the designs perform as expected. However, the scope of these empirical design techniques is limited to the input of the regression model: an intelligent designer will not use a general aviation weight model for a transport category aircraft. To this end, there is a significant lack of small UAV-class guidelines for designing an aircraft.

Accurate estimations of a small-scale vehicle's lift and drag characteristics are extremely critical to the aircraft designer, and affect both point performance (turn rates, climb rates, stall speeds, etc.) and mission performance (range and endurance). Many of the prediction tools available in the classic lift and drag textbooks from Hoerner ${ }^{[4],[8]}$ only apply to larger scale structures. In addition, drag prediction is extremely difficult on small vehicles, as some sources of drag are not easily modeled. For instance, actuator control horns and protruding screw heads are not typically included in the CFD analysis of aircraft. However, for small vehicles, these sources of "crud" drag can be a significant portion of the total vehicle's drag value.

One advantage of small UAVs is that they are fairly inexpensive, which makes building multiple fully-functioning prototypes a viable option. The author chose to take advantage of this fact and develop a flight data acquisition system with a primary goal of measuring the lift and drag characteristics of a small UAV. This would enable vehicle designers to build a conceptual-design-level prototype, and both develop and validate predictive, regression-based models. The system would also allow designers to conduct quantitative trade studies, such as the trade between drag reduction technologies (wheel pants, retractable landing gear, winglets, etc.) and the weight associated with them. 
The author also desired additional sensors to aid designers with more than just lift and drag characteristics. Some areas of interest are estimating stability and control derivatives, as well as possible control algorithm testing, in-flight thrust measurement, and payload integration capabilities. To accomplish these goals, sensors not directly necessary to lift and drag estimation were included in the overall system. The system was also designed in a manner that made it reconfigurable. This allows future designers to decide what combination of accuracy, risk, and sensing capabilities they need on a given flight test, and to then package the sensors in a manner that meets vehicle integration requirements.

For this paper, the author chose to integrate the necessary sensors to estimate lift and drag forces, as well as those necessary for a basic INS, ambient temperature measurement, and servo and motor signals. The system will be validated by measuring the as-built drag polar of an R/C aircraft, as well as all other available states the system is capable of measuring. 


\subsection{Method}

Some basic assumptions will apply throughout the modeling of dynamics in this paper. They are as follows:

1. The vehicle is a fixed mass.

2. Coriolis effects are negligible.

3. Thrust will be assumed to be 0 .

Note that a stationary atmosphere is not assumed. The fixed mass assumption is consistent with the electric aircraft used to test the system. At the altitude and speed at which the vehicles will be tested, Coriolis effects can be ignored ${ }^{[9]}$. The zero-thrust assumption is in place to minimize drag error. Any error in a measured state will decrease the accuracy of the drag measurement, and the accuracy of a given drag measurement can be no better than the worst state measurement error. In-flight thrust is difficult to measure accurately, so a folding propeller will be used, and the motor will be turned off during data acquisition. This will allow the propeller to fold back, eliminating most of the wind-mill drag associated with a stalled propeller.

\subsection{Reference Frames}

For this thesis, the reference frames used will follow standard convention, ${ }^{[9]}$ which will be repeated here for clarity.

\subsubsection{North-East-Down (NED) Axes $\left(x_{n e d}, y_{n e d}, z_{n e d}\right)$}

The NED axis system defines a local tangent plane on the Earth's surface, with the origin coinciding with the vehicle's center of gravity. The $\hat{i}$ vector points due north, the $\hat{j}$ vector 
points due east, and the $\hat{k}$ vector points towards the center of the earth, in accordance with the right-hand rule. This coordinate system is vehicle carried, meaning the origin is fixed to the aircraft, but the axis directions are independent of vehicle orientation.

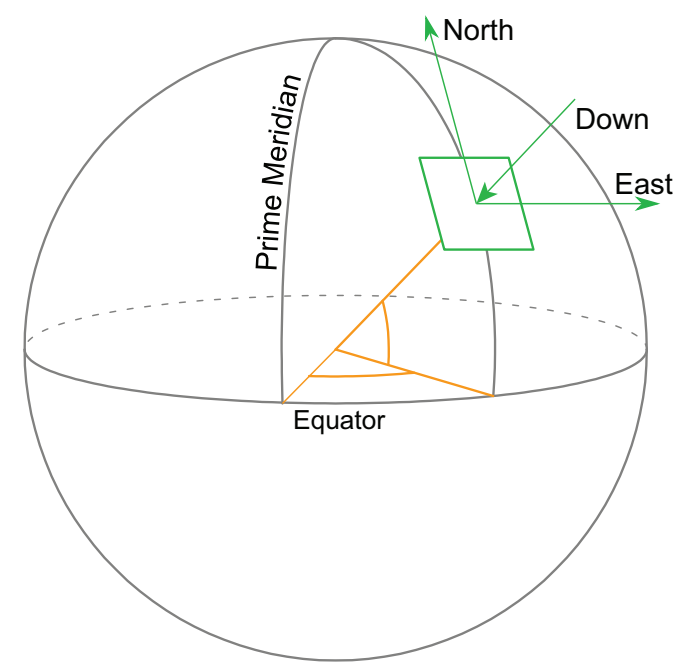

Figure 2.1: NED Frame of Reference ${ }^{[1]}$

\subsubsection{Body Axes $\left(x_{b}, y_{b}, z_{b}\right)$}

The body axis system has its origin at the vehicle's center of gravity, with the $\hat{i}$ direction pointing out the vehicle's nose, the $\hat{j}$ direction pointing out the right wing, and the $\hat{k}$ direction pointing out the belly of the aircraft, in accordance with the right-hand rule. This coordinate frame is fixed to the body, meaning the aircraft's spatial orientation does not change the direction of the axes. 

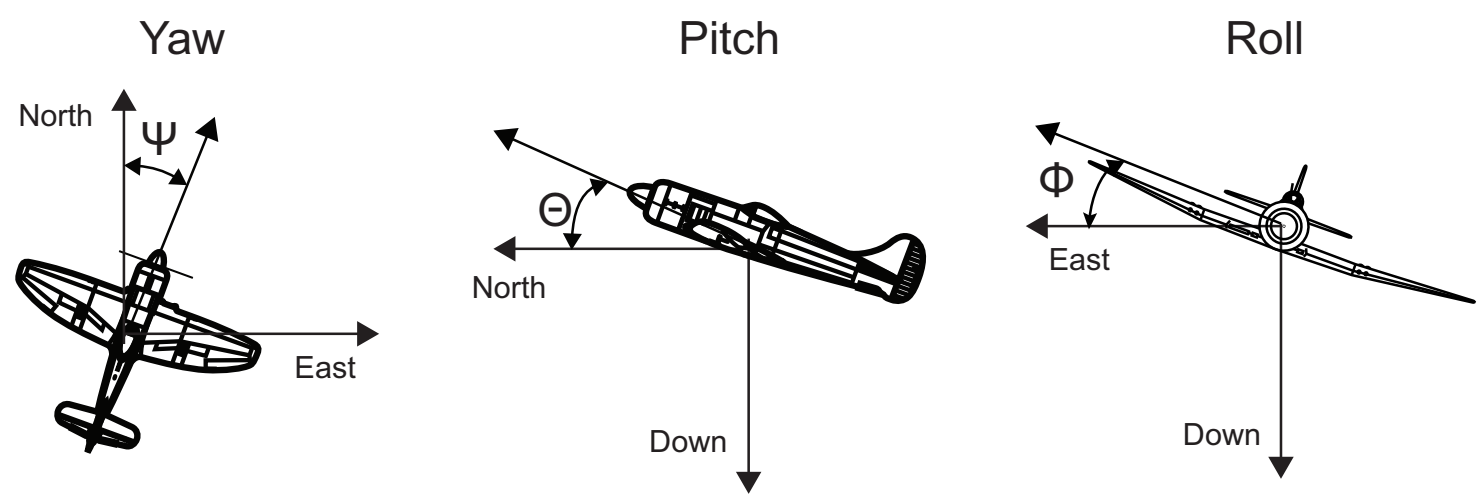

\section{Figure 2.2: Body Axes Definition ${ }^{[2]}$}

\subsubsection{Stability Axes $\left(x_{s}, y_{s}, z_{s}\right)$}

The stability axes are defined with its origin coinciding with the center of gravity of the vehicle. This axis system has essentially the same directions as the body axes, except rotated about the body axis $\hat{j}$ through an initial angle-of-attack, $\alpha_{0}$. This inital angle-of-attack is defined at the beginning of a test maneuver and is then set for the remainder of the test, making it a body-fixed coordinate system. This system assumes no initial sideslip angle ${ }^{[10]}$. In Figure 2.3, only the $\vec{i}$ stability vector is shown, for clarity.

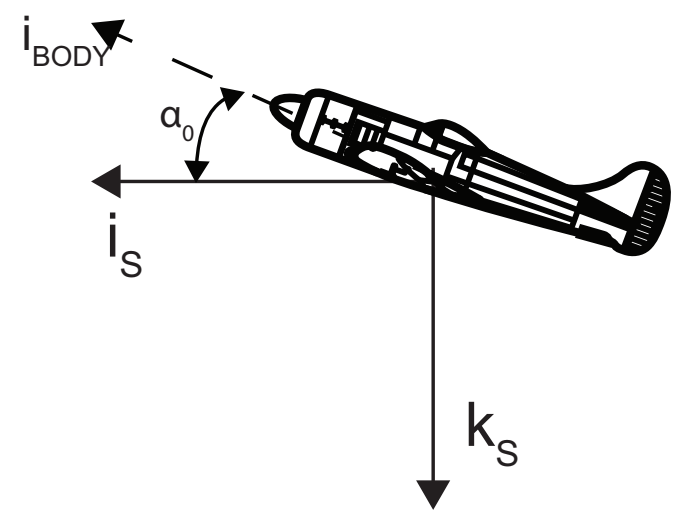

Figure 2.3: Stability Axes Definition 


\subsubsection{Wind Axes $\left(x_{w}, y_{w}, z_{w}\right)$}

The wind axes are, again, a vehicle-carried coordinate system, meaning the origin of the wind axis also coincides with the center of gravity of the vehicle. However, the wind axes are not a body-fixed coordinate frame. The $\hat{i}$ direction points into the oncoming air, as seen from the vehicle. The $\hat{k}$ direction lies in the $\mathrm{x}$-z plane of the body reference frame. The $\hat{j}$ direction is then defined to be out the right side of the vehicle, in order to follow the right hand rule.

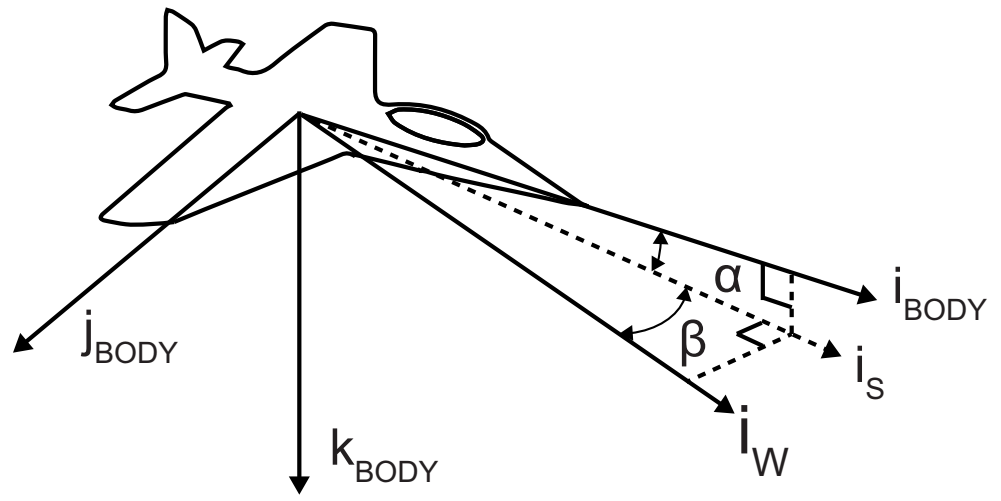

Figure 2.4: Wind Axes Definition

In Figure 2.4, only the $\vec{i}$ wind vector is shown, for clarity.

\subsection{Equations of Motion}

Newton's 2nd Law of Motion states

$$
\Sigma \vec{F}=\frac{d}{d t}(m \vec{V})
$$

where $\Sigma \vec{F}$ is the sum of all applied forces, $\vec{m}$ is the mass of the vehicle, and $\vec{V}$ is the vehicle's velocity. Using the fixed mass assumption, this reduces to

$$
\begin{aligned}
\Sigma \vec{F} & =m \frac{d \vec{V}}{d t} \\
& =m \vec{a}
\end{aligned}
$$


The applied forces on the vehicle for drag polar estimation are

$$
\Sigma \vec{F}=\vec{F}_{A}+\vec{F}_{G}+\vec{F}_{T}
$$

where $\overrightarrow{F_{A}}$ accounts for all aerodynamic forces acting on the vehicle, $\vec{F}_{G}$ is the force due to gravity, and $\vec{F}_{T}$ accounts for forces from the propulsion system, which are assumed to be zero.

The aerodynamic forces lift and drag are described in the wind reference frame. In general, they are defined as

$$
\vec{F}_{A_{w}}=D \hat{i}_{w}+Y \hat{j}_{w}+L \hat{k}_{w}
$$

where $D$ is drag force, $Y$ is side force, and $L$ is lift force.

The gravitational force on the vehicle acts in the $+z_{N E D}$ direction and is equal in magnitude to the vehicle's weight $W$, leading to

$$
\vec{F}_{G_{N E D}}=0 \hat{i}_{N E D}+0 \hat{j}_{N E D}+W \hat{k}_{N E D}
$$

The aerodynamic and gravity forces can be combined and expressed in the body frame

$$
m \vec{a}_{b}=\vec{F}_{G_{b}}+\vec{F}_{A_{b}}
$$

which can then be expressed as

$$
\begin{aligned}
& m \vec{a}_{b}-m \vec{g}=\vec{F}_{A_{b}} \\
& m\left(\vec{a}_{b}-\vec{g}\right)=\vec{F}_{A_{b}}
\end{aligned}
$$

Then, it is noted that a body mounted accelerometer will not measure $\vec{a}_{b}$, but will instead measure the quantity $\vec{g}-\vec{a}_{b}$. This means Equation 2.2 .9 is really

$$
\vec{F}_{A_{b}}=-m \vec{r}_{b}
$$

where $\vec{r}_{b}$ is the reading from a body mounted accelerometer. The aerodynamic forces in wind axes, which is the goal, can then be calculated using a rotation matrix

$$
\vec{F}_{A_{w}}=\bar{R}_{w}^{b} \vec{F}_{A_{b}}
$$


Equations 2.2.10 and 2.2.11 are important, and show that the only sensors necessary for drag polar estimation during gliding flight are a body-mounted accelerometer and an air data system.

\subsection{Kalman Filter Usage}

This paper examined using multiple Kalman filters to estimate both regression coefficients and improved states. By the end, it was not used for improving the state estimation, since neither Euler angles or accelerations were available. It was studied for a robust regression coefficient estimator, which will be discussed more in Section 5.3. As a brief overview, the Kalman filter combines the measured state with a predicted state to give an optimal ${ }^{[11]}$ estimate of the actual system state. As an example, if a car is traveling at $10 \mathrm{ft} / \mathrm{s} \pm 1 \mathrm{ft} / \mathrm{s}$, after one second it is expected to be 10 feet away. If it is measured to be 14 feet away, \pm 5 foot, the Kalman filter will average the estimated $10 \mathrm{ft}$ with the measured 14 feet, based on how accurately each is known.

\subsubsection{Linear Kalman Filter}

A linear Kalman filter can be applied ${ }^{[12]}$ where the system in question can be described in the form

$$
x_{k}=\bar{A} x_{k-1}+\bar{B} u_{k-1}+w_{k-1}
$$

where $\bar{A}$ is the state transition matrix, $x_{k-1}$ is the previous state, $\bar{B}$ is the input matrix, $u_{k-1}$ is the input vector, and $w_{k-1}$ is random process noise.

The measured state is then

$$
z_{k}=\bar{H} x_{k}+v_{k}
$$

where $\bar{H}$ is the output matrix and $v_{k}$ is measurement noise.

The Kalman filter operates in a predictor-corrector manner, where the predictor step is often 
called the a priori estimate, and the corrector step is often called the a posteriori estimate. The a priori state estimate is calculated using prior states and inputs, while assuming no process noise

$$
x_{k}^{-}=\bar{A} x_{k-1}+\bar{B} u_{k-1}
$$

The a priori estimate of the covariance matrix is projected in a similar manner

$$
\bar{P}_{k}^{-}=\bar{A} \bar{P}_{k-1} \bar{A}^{T}+\bar{Q}
$$

where $\bar{P}$ is the covariance matrix and $\bar{Q}$ is the process noise matrix.

The Kalman gain is calculated by combining the predicted, a priori covariance matrix with the measurement noise covariance matrix $\bar{R}$

$$
\bar{K}_{k}=\bar{P}_{k}^{-} \bar{H}^{T}\left(\bar{H} \bar{P}_{k}^{-} \bar{H}^{T}+\bar{R}\right)^{-1}
$$

This optimal Kalman gain is then used to estimate the a posteriori estimate of both the state and the covariance matrix

$$
\begin{gathered}
x_{k}=x_{k}^{-}+\bar{K}_{k}\left(z_{k}-y_{k}\right) \\
\bar{P}_{k}=\left(\bar{I}-\bar{K}_{k} \bar{H}\right) \bar{P}_{k}^{-}
\end{gathered}
$$

where $y_{k}$ is the predicted value of $z_{k}$ found using the output matrix $\bar{H}$ and the a priori state estimate

$$
y_{k}=\bar{H} x_{k}^{-}
$$

Note that Equation 2.3.6 is essentially a weighted average of a measured state and an expected state. The weighting is the Kalman gain, which is related to the ratio of confidence in the measured state and the expected state. For a 1-D case with equal confidence between the measured state and the expected state, the Kalman gain $\bar{K}_{k}=0.5$, and the Kalman filter becomes a simple mean. 


\subsubsection{Extended Kalman Filter}

The Extended Kalman filter is used for a non-linear system and is essentially a linearization of a nonlinear plant. A nonlinear system can be described as ${ }^{[12]}$

$$
\begin{aligned}
& x_{k}=f\left(x_{k-1}, u_{k-1}, w_{k-1}\right) \\
& z_{k}=h\left(x_{k}, v_{k}\right)
\end{aligned}
$$

The process noise $w_{k-1}$ and measurement noise $v_{k}$ are not known (or the Kalman filter would not be necessary), so the states are approximated assuming both noise sources are zero, as shown in Equation 2.3.11

$$
\begin{aligned}
& \tilde{x}_{k}=f\left(x_{k-1}, u_{k-1}, 0\right) \\
& \tilde{z}_{k}=h\left(\tilde{x}_{k}, 0\right)
\end{aligned}
$$

where $\tilde{x}_{k}$ is the approximate state vector and $\tilde{z}_{k}$ is the approximate measurement vector.

The actual states are related to the approximate states by

$$
\begin{aligned}
& x_{k} \approx \tilde{x}_{k}+\bar{A}\left(x_{k}-x_{k-1}\right)+\bar{W} w_{k-1} \\
& z_{k} \approx \tilde{z}_{k}+\bar{H}\left(x_{k}-x_{k-1}\right)+\bar{V} v_{k}
\end{aligned}
$$

where the matrices $\bar{A}, \bar{W}, \bar{H}$, and $\bar{V}$ represent the different Jacobian matrices:

$$
\begin{aligned}
\bar{A} & =\frac{\partial f_{i}}{\partial x_{j}}\left(x_{k-1}, u_{k-1}, 0\right) \\
\bar{W} & =\frac{\partial f_{i}}{\partial w_{j}}\left(x_{k-1}, u_{k-1}, 0\right) \\
\bar{H} & =\frac{\partial h_{i}}{\partial x_{j}}\left(x_{k}, 0\right) \\
\bar{V} & =\frac{\partial h_{i}}{\partial v_{j}}\left(x_{k}, 0\right) .
\end{aligned}
$$

The Extended Kalman filter uses these linearized equations to perform the same process as the linear Kalman filter. Again, the first step is to calculate the a priori estimate of the 
state and the covariance matrix

$$
\begin{aligned}
& x_{k}^{-}=f\left(x_{k-1}, u_{k-1}, 0\right) \\
& \bar{P}_{k}^{-}=\bar{A}_{k} \bar{P}_{k-1} \bar{A}_{k-1}^{T}+\bar{W}_{k} \bar{Q}_{k-1} \bar{W}_{k}^{T} .
\end{aligned}
$$

Next, the Kalman gain is calculated

$$
\bar{K}_{k}=\bar{P}_{k}^{-} \bar{H}_{k}^{T}\left(\bar{H}_{k} \bar{P}_{k}^{-} \bar{H}_{k}^{T}+\bar{V}_{k} \bar{R}_{k} \bar{V}_{k}^{T}\right)^{-1}
$$

The Kalman gain is then used to calculate the a posteriori estimate of the state and covariance matrix

$$
\begin{aligned}
& x_{k}=x_{k}^{-}+\bar{K}_{k}\left(z_{k}-y_{k}\right) \\
& \bar{P}_{k}=\left(\bar{I}-\bar{K}_{k} \bar{H}_{k}\right) \bar{P}_{k}^{-}
\end{aligned}
$$

where $y_{k}$ is, as in the linear case, the predicted value of $z_{k}$, but calculated using the nonlinear output function and the a priori state estimate

$$
y_{k}=h\left(x_{k}^{-}, 0\right)
$$

Unlike the linear Kalman filter, the Extended Kalman filter is not proven to be optimal. However, it has been utilized for a wide range of applications with excellent results. 


\subsection{Drag Meta-Modeling}

Drag force comes from many different contributions, but can be split into drag that is independent of lift, and drag that is due to lift ${ }^{[13]}$. The summation of all sources of drag that are independent of lift is often called minimum drag $\left(C_{D_{\min }} \text { often used for the coefficient }\right)^{[5]}$, and is roughly constant, for a given Reynold's number and Mach number. The drag due to lift can be split into viscous drag-due-to-lift and inviscid drag-due-to-lift.

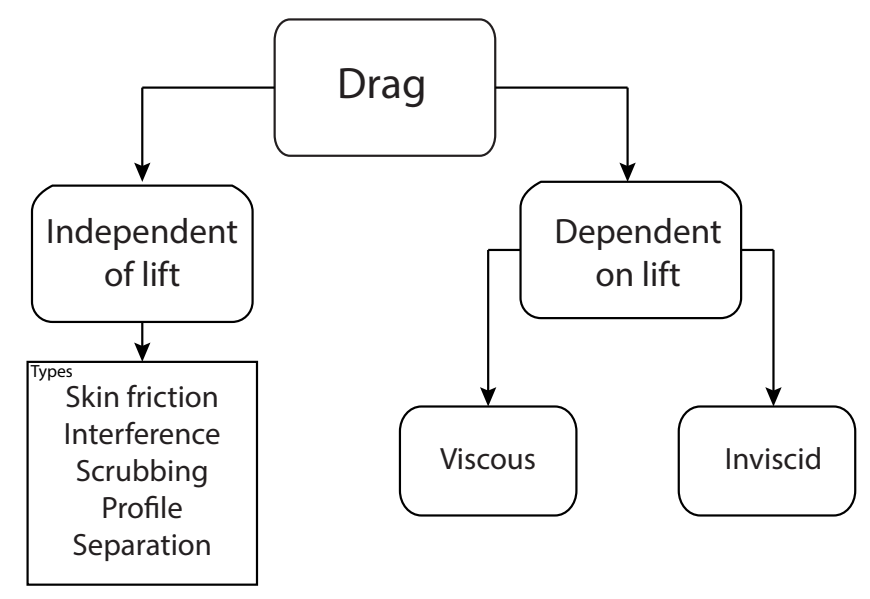

\section{Figure 3.1: Drag Contribution Types}

The viscous drag-due-to-lift is a profile drag, and it increases when the angle of attack of the wing increases, therefore generating more lift. It is a function of airfoil geometry, such as leading edge radius, thickness distribution, and camber. This type of drag is independent of finite wing vortices, and can be seen on two-dimensional airfoil data charts. To show an example of viscous drag-due-to-lift, a NACA 4412 was analyzed using XFOIL ${ }^{[14]}$.

Figure 3.3 shows the airfoils viscous drag-due-to-lift. Since the shape is roughly parabolic through the linear region of the lift curve, the contribution to the total aircraft drag is approximated using the Equation 3.0.1 ${ }^{[13]}$.

$$
C_{D_{V i s c . \text { iff }}}=K_{1}\left(C_{L}-C_{L_{M i n \text { Drag }}}\right)^{2}
$$




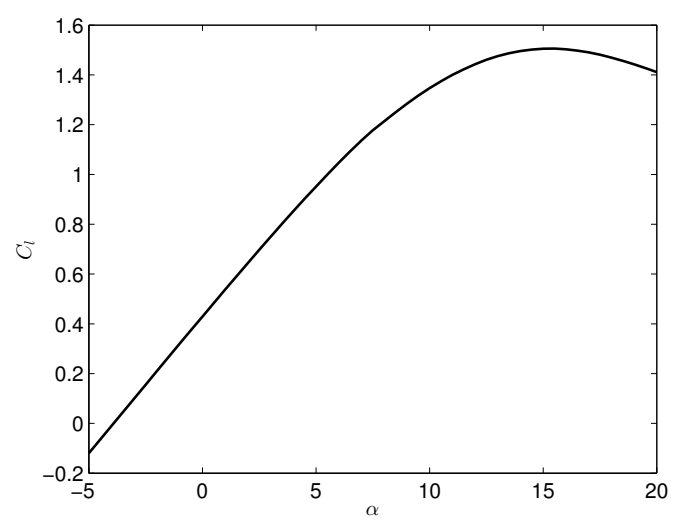

Figure 3.2: NACA 4412 Lift Curve

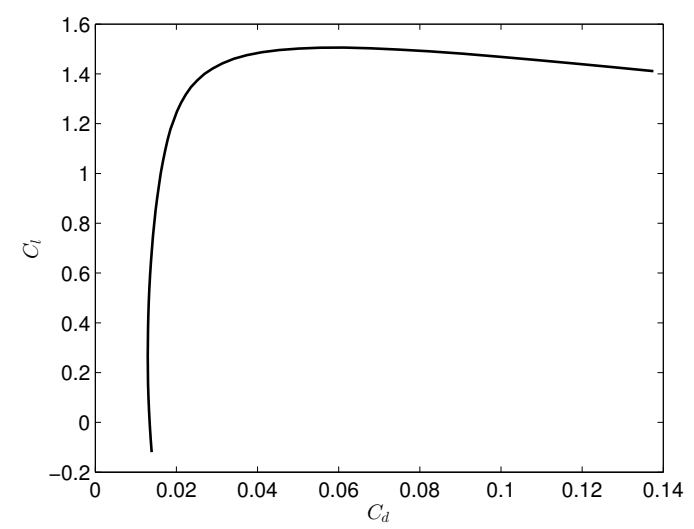

Figure 3.3: NACA 4412 Drag Polar

where $K_{1}$ is the slope of the line shown in Figure 3.4, and $C_{L_{\text {Min Drag }}}$ is the lift coefficient at which minimum drag occurs (roughly 0.25 for this airfoil.)

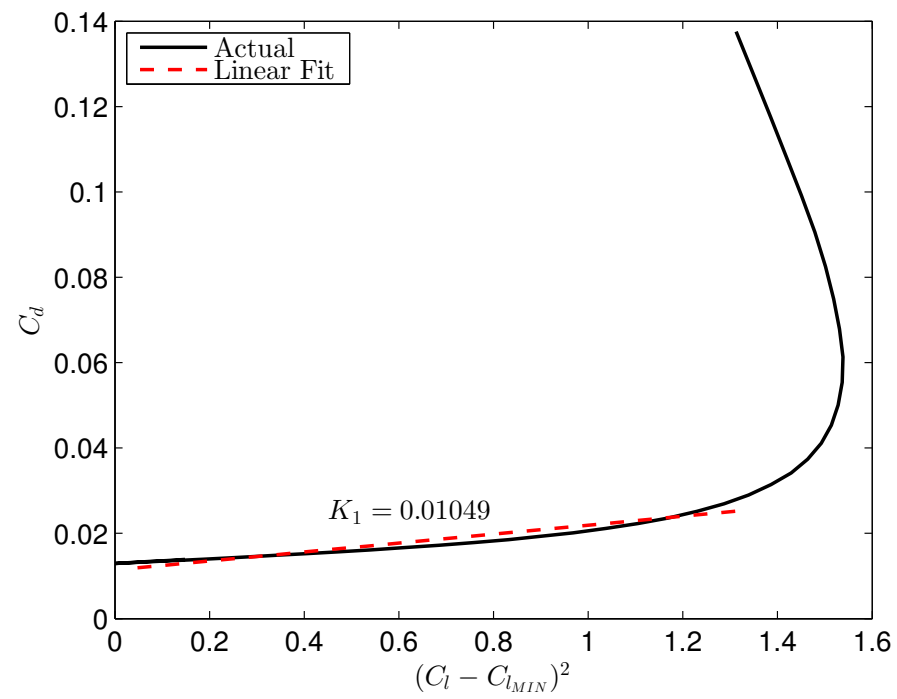

Figure 3.4: NACA $4412 K_{1}$ Estimation

Inviscid drag-due-to-lift occurs because of the pressure difference between the top and bottom of a finite wing. This pressure difference causes the high pressure flow underneath the wing to slip over to the top of the wing, causing a downwash velocity on the free stream velocity, shown in Figure 3.5. 


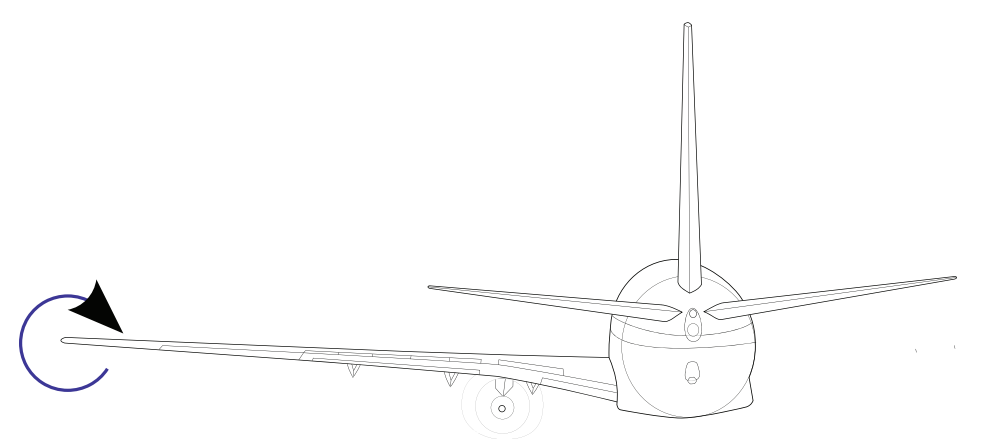

Figure 3.5: Downwash Caused By Wingtip Vortices ${ }^{[3]}$

This downwash changes the direction of the flow going into the airfoil. Since lift acts perpendicularly to the flow going into airfoil, there will be an induced angle between the free-stream lift vector (perpendicular to $V_{\infty}$ ) and the airfoil's lift vector.

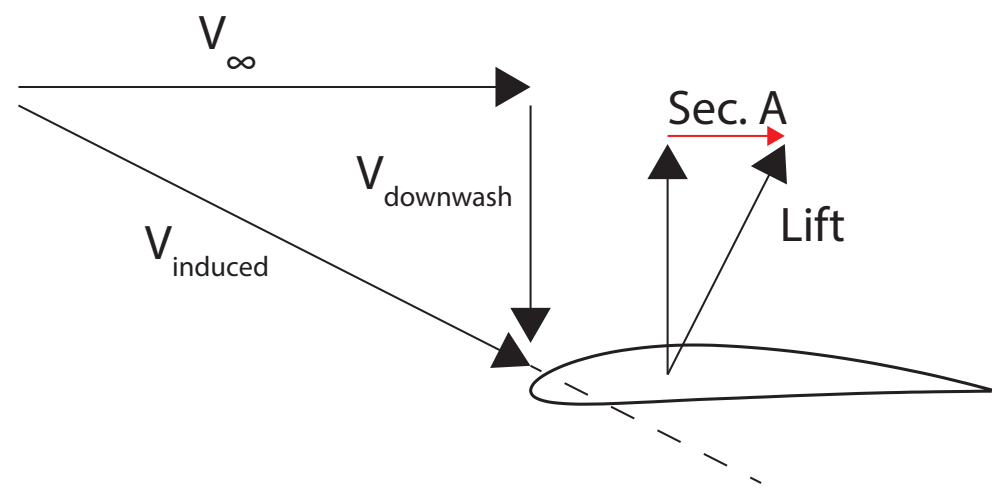

\section{Figure 3.6: Induced Drag Free Body Diagram}

The red section, labeled Sec. A in Figure 3.6, is a component of the airfoil's lift which is parallel to the free stream velocity, $V_{\infty}$, meaning the airfoil's lift causes drag on the vehicle.

Induced drag is affected by the span lift distribution and the wing aspect ratio ${ }^{[15]}$, and is governed by Equation 3.0.2.

$$
C_{D_{i}}=\frac{C_{L}^{2}}{\pi e A R}=K_{2} C_{L}^{2}
$$

These three coefficients $\left(C_{D_{\text {min }}}, K_{1}\right.$, and $\left.K_{2}\right)$ are combined to result in a parabolic drag polar of the form 


$$
C_{D}=C_{D_{\min }}+K_{1}\left(C_{L}-C_{L_{M I N}}\right)^{2}+K_{2} C_{L}^{2} .
$$

This drag polar gives valuable insight into how a vehicle will perform. The complete drag polar can be found using various regression techniques, discussed in the following sections.

\subsection{Regression Model - Least Squares Fit}

The standard model for a polynomial regression is

$$
\hat{y}=\beta_{0}+\beta_{1} x+\beta_{2} x^{2}+\ldots
$$

where $\hat{y}$ is the estimate of the dependent variable of the regression model, $\beta_{i}$ is the $i-t h$ regression coefficient, and $x$ is the independent regression variable.

When Equation 3.0.3 is expanded and like-terms of $C_{L}$ are combined, equation 3.1.1 becomes

$$
C_{D}=\left(C_{D_{m i n}}+K_{1} C_{L_{m i n}}^{2}\right)-2 K_{1} C_{L_{m i n}} C_{L}+\left(K_{1}+K_{2}\right) C_{L}^{2}
$$

The value $\left(C_{D_{\min }}+K_{1} C_{L_{m i n}}^{2}\right)$ is referred to as the parasite drag coefficient, and is represented by $C_{D_{0}}{ }^{[5]}$.

These coefficients can be estimated using an Ordinary Least Squares fit. The OLS problem statement is as follows

$$
\bar{A} \vec{x}=\vec{b}
$$

If $\bar{A}$ is an $m \mathrm{x} n$ matrix of measured state data, $\vec{x}$ is an $n \mathrm{x} 1$ vector of correlation coefficients, and $\vec{b}$ is an $m \times 1$ vector of measured function data, the solution to the OLS problem is

$$
\begin{aligned}
\bar{A} \vec{x} & =\vec{b} \\
\bar{A}^{T} \bar{A} \vec{x} & =\bar{A}^{T} \vec{b} \\
\left(\bar{A}^{T} \bar{A}\right)^{-1}\left(\bar{A}^{T} \bar{A}\right) \vec{x} & =\left(\bar{A}^{T} \bar{A}\right)^{-1} \bar{A}^{T} \vec{b} \\
\bar{I} \vec{x} & =\left(\bar{A}^{T} \bar{A}\right)^{-1} \bar{A}^{T} \vec{b} .
\end{aligned}
$$


When applied to estimating a parabolic drag polar, the $\bar{A}$ matrix becomes

$$
\bar{A}_{i,:}=\left[\begin{array}{lll}
1 & C_{L_{i}} & C_{L_{i}}^{2}
\end{array}\right]
$$

the $\vec{b}$ vector becomes

$$
\vec{b}_{i}=\left[C_{D_{i}}\right]
$$

and the $\vec{x}$ vector, which is the vector of interest, becomes

$$
\vec{x}=\left[\begin{array}{lll}
C_{D_{0}} & -2 K_{1} C_{L_{\text {min }}} & \left(K_{1}+K_{2}\right)
\end{array}\right]^{T} .
$$

The coefficients $C_{D_{0}}, K_{1}$, and $K_{2}$ can then be found, assuming $C_{L_{m i n}}$ is known through wind tunnel testing, XFOIL, CFD, or other means.

\subsection{Regression Model - Kalman Filter}

The coefficients in question can also be estimated using an Extended Kalman filter. The system can again be described as

$$
C_{D}=C_{D_{0}}-2 K_{1} C_{L_{m i n}} C_{L}+\left(K_{1}+K_{2}\right) C_{L}^{2}
$$

For the Kalman filter regression, the state to be estimated are the coefficients $C_{D_{0}}$, $-2 K_{1} C_{L_{m i n}}$, and $K_{1}+K_{2}$. For ease of notation, substitute

$$
\begin{aligned}
& C_{1}=-2 K_{1} C_{L_{\text {min }}} \\
& C_{2}=K_{1}+K_{2} .
\end{aligned}
$$

Since the regression coefficients should not change, the state transition matrix is an identity matrix, leading to 


$$
\begin{aligned}
\hat{x}_{k} & =\left[\begin{array}{lll}
C_{D_{0}} & C_{1} & C_{2}
\end{array}\right] \\
A & =\left[\begin{array}{lll}
1 & 0 & 0 \\
0 & 1 & 0 \\
0 & 0 & 1
\end{array}\right] .
\end{aligned}
$$

The measured data $z_{k}$ is a vector containing the lift and drag coefficients at the $k$-th instant in time

$$
z_{k}=\left[\begin{array}{l}
C_{D_{k}} \\
C_{L_{k}}
\end{array}\right]=h\left(x_{k}, 0\right)
$$

The vector $y_{k}$ contains the estimates of $C_{D_{k}}$ and $C_{L_{k}}$ found using the a priori state vector $\hat{x}_{k}^{-}$and is equal to

$$
y_{k}=\left[\begin{array}{c}
C_{D_{0}}^{-}+C_{1}^{-} C_{L}+C_{2}^{-} C_{L}^{2} \\
C_{L}
\end{array}\right]=h\left(x_{k}^{-}, z_{k}, 0\right)
$$

To implement into the Extended Kalman filter, the Jacobian of $h\left(x_{k}^{-}, z_{k}, 0\right)$ with respect to $x_{k}$ needs to be calculated. Once done, the $H$ matrix in the EKF becomes

$$
H_{k}=\left[\begin{array}{ccc}
1 & C_{L_{k}} & C_{L_{k}}^{2} \\
0 & 0 & 0
\end{array}\right]
$$

With the $H$ matrix calculated, the EKF algorithim can be implemented as described in Section 2.3.2. The measurement noise covariance at each instant was calculated by doing error propagation as described in Section 4.1 and the process noise covariance was set to zero because the parabolic regression coefficients should be exactly constant. 


\subsection{Error Analysis}

Without estimates of the error in data, the data itself is fairly meaningless. There are two main types of error in the system: model regression error and the error of a single data point. The error of a single data point comes from random noise in sensors, and can be decreased by improving the accuracy of the sensor or filtering the results. The model regression error comes from the fact that every aspect of the dynamics of the system are not precisely modeled. This type of error can be reduced by improving the accuracy of the dynamics being modeled, choosing a better regression model, or by increasing the number of data points collected, discussed later.

\subsection{Random Error}

The equations of motion can be used to propagate uncertainty in a signal, and they allow the uncertainty in the coefficients to be estimated based on sensor noise.

The error of a single point is assumed to be random and normally distributed. The first step in error propagation is to define $y_{i}$ to be the $i$-th entry of the true function vector, $\hat{y}_{i}$ to be the $i$-th entry of the measured function vector, and to then do a Taylor series expansion about the operating point.

$$
\hat{y}_{i}=y_{i}+\frac{\partial y_{i}}{\partial x_{j}} d x_{j}
$$

where $x_{j}$ is the $j$-th element of the state vector. Note that the term $\frac{\partial y_{i}}{\partial x_{j}}$ is the Jacobian matrix $\left(J_{i j}\right)$ of the state transition function. The error can then be defined as

$$
d y_{i}=\hat{y}_{i}-y_{i}=J_{i j} d x_{j}
$$

If the error is then interpreted as a discrete difference instead of a continuous difference, 
Equation 4.1.2 becomes

$$
\Delta y_{i}=J_{i j} \Delta x_{j}
$$

If the $\Delta$ values are further assumed to represent standard deviations of normally distributed error, Equation 4.1.3 becomes

$$
\sigma_{y_{i}}=\sqrt{J_{i j}^{2} \sigma_{x_{j}}^{2}}
$$

For the purposes of this research, $\sigma_{y_{i}}$ is a vector of the standard deviations of the aerodynamic force coefficients,

$$
\sigma_{y_{i}}=\left[\begin{array}{lll}
\sigma_{C_{D_{i}}} & \sigma_{C_{Y_{i}}} & \sigma_{C_{L_{i}}}
\end{array}\right]^{T}
$$

and $\sigma_{x_{j}}$ is a vector of the standard deviations of the state values,

$$
\sigma_{x_{j}}=\left[\begin{array}{lll}
\sigma_{r_{b_{j}}} & \sigma_{\alpha_{j}} & \sigma_{\beta_{j}}
\end{array}\right]^{T} .
$$

For initial error estimation, the noise levels reported by instrument manufacturers was assumed to be one standard deviation of a normal distribution with mean equal to zero. The Jacobian matrix was calculated at each observed data point and combined with the sensors' standard deviation to produce estimates of the standard deviations of the aerodynamic coefficients.

One of the key findings of this section is that the error of the coefficient depends on the coefficient itself. This means that the error varies from data point to data point, which is called heteroskedasticity (as opposed to homoskedasticity, which means the error is independent of the state itself). To account for this, an error estimate function was created in MATLAB, which used the error propogation outlined in this section. This function was used whenever an estimate of point error was required, such as for the variance matrix $P_{k}$ used in the Kalman filters utilized for state estimation. A plot of simulated flight data is shown in Figure 4.1, where the error bounds shown were calculated using the error estimate function. Note that this figure also shows the heteroskedastic nature of the error. 


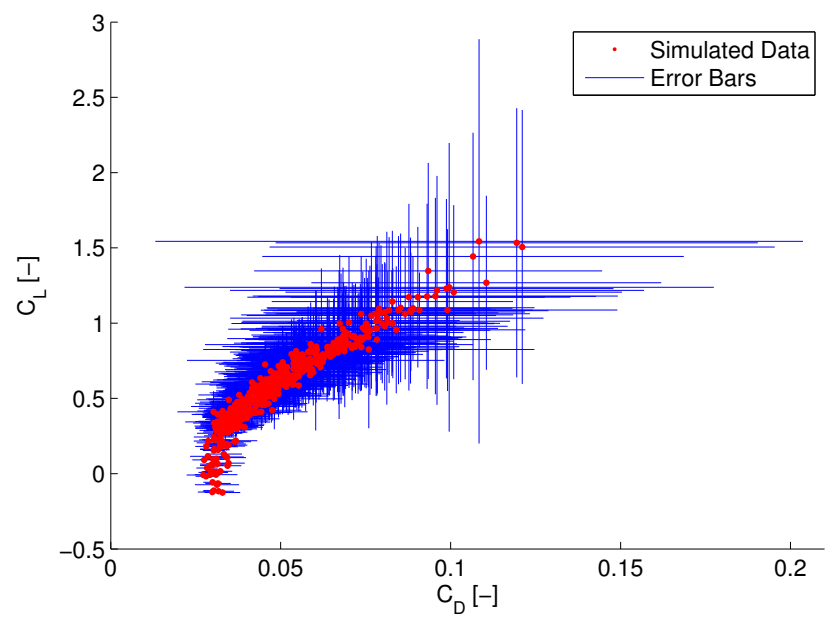

Figure 4.1: Heteroskedastic Error from Simulated Flight

While heteroskedasticity does not color the estimate of $\hat{\beta}_{i}$, it does color the confidence intervals. The method of dealing with this problem is discussed in Section 4.2.

\subsection{Least Squares Regression Error}

The error of a single data point is not the main driving factor in the accuracy of a regression model. The important factor in the model is how accurately the coefficients are known, which is a function of the accuracy of each point, as well as the number of points sampled. The main parameter that describes the accuracy of the regression coefficients are the confidence intervals. A confidence interval is a range of values such that, if the experiment were repeated, the parameter calculated would be within the range some percentage of the time.

A parameter can be represented as an estimated value, with a confidence bound

$$
\beta=\beta_{E S T} \pm t \frac{\sigma}{\sqrt{n}}
$$

where $\beta$ is the parameter in question, $\beta_{E S T}$ is the estimated value of the parameter, $t$ is the Student's $t$ value based on the number of samples and the desired confidence interval, $\sigma$ 
is the standard deviation of the sample, and $n$ is the number of data points collected. Since the number of data points collected during flight will be large $(n>100)$, the $t$ value will be taken as 1.96 for a $95 \%$ confidence interval.

As previously mentioned, one of the assumptions made in a least squares regression is homoskedasticity. However, the error using standard uncertainty propagation is heteroskedastic. This becomes a problem in estimating confidence intervals, because the standard error can be driven by outliers. If each data point had the same error, these outliers could be valid. However, if the data is heteroskedastic, the outlier may have larger error bounds (see Figure 4.1,) meaning the data point is not as likely as it first appears. This fact can drive the standard error estimate to be larger than is appropriate, which leads to a larger confidence interval and possibly a false lack of rejection of the confidence interval's hypothesis test.

To account for this, the robustfit function in MATLAB was used to estimate both the regression coefficients and robust standard error estimates. The robustfit function calculates heteroskedastically-robust standard error estimates by doing an iterative weighted least squares, where the weighting is based on a radial basis function from the previous least squares solution. This means that the farther away a data point is from the estimated regression model, the less impact it has on the standard error of the model. The default weighting function used by robustfit is bisquare, and it was used for this research.

\subsection{Kalman Filter Regression Error}

Kalman filters are often used to propagate states. The filter does this by combining the system dynamics with a measured state. The variance is propagated using Equation 2.3.23. When estimating coefficients using the Kalman filter, the $\bar{A}$ state transition matrix is an identity matrix, which is due to the fact that the coefficients stay constant. When propagated through the filter, this means the state estimate $x_{k}$ is essentially a variance-weighted-average of the coefficient estimates. The matrix $P_{k}$ contains the variance of the coefficient estimates. 
Equation 4.2.1 calculates the confidence interval of regression coefficients and needs the standard deviation of the mean, also called the standard error. The matrix $P_{k}$ can be used to calculate the confidence intervals by noting

$$
\bar{P}_{k}=\left[\begin{array}{cccc}
\sigma_{1}^{2} & 0 & \ldots & 0 \\
0 & \sigma_{2}^{2} & \ldots & 0 \\
\vdots & \vdots & \ddots & \vdots \\
0 & 0 & \ldots & \sigma_{i}^{2}
\end{array}\right] .
$$

The confidence interval for coefficient $\beta_{i}$ can be calculated using $\sigma_{i}$ in Equation 4.2.1. 


\subsection{Simulation}

A 6-DOF flight simulator was used to validate the drag prediction method before hardware was purchased. The main utility of the simulator was to provide simulated flight test data with signals that contained no errors. The actual sensors used for flight testing contain noise, and this noise can be added onto the pure simulator signals to test the sensitivity of the drag polar regression to sensor accuracy.

\subsection{Simulation Environment}

The flight simulator used was a model of the de Haviland Beaver that comes as a demo in the Aerospace Toolbox of Simulink. The Simulink model was modified to output required signals to the workspace, which essentially created a sensor with zero noise. The mass, moments of inertia, and reference lengths were then scaled to those of a Zagi R/C aircraft ${ }^{[16]}$. The original Simulink model was already connected to a FlightGear Flight Sim, used as a visualization engine. This model was slightly altered to make flight gauges function properly.

\subsection{Simulation Inputs}

The engine forces and moments were set to zero in the simulator, to match the assumption of a folding propeller. The drag force calculation built into the Beaver Simulink model was replaced with a parabolic drag polar of the form

$$
C_{D}=C_{D_{0}}+K_{1}\left(C_{L}(\alpha)-C_{L_{m i n}}\right)^{2}+\frac{\left(C_{L}(\alpha)\right)^{2}}{\pi e A R}
$$

Airfoil data, including $K_{1}, C_{L_{m i n}}$, and $C_{L}(\alpha)$, was taken from nonlinear aerodynamic data of a NACA 0012 ${ }^{[17]}$. While this approximation to a real drag polar does not capture the nonlinear section of profile drag rise due to stall, it does represent the limited lifting capability of a real wing. 


\subsection{Simulation Results}

The first goal of the simulation testing was to verify the drag polar equations were correct, and that the data analysis routines developed in MATLAB did in fact match inputs to outputs. The simulation was initialized with various initial states to ensure there was no dependency on initial conditions. The vehicle was then flown by an $\mathrm{R} / \mathrm{C}$ aircraft pilot using a joystick attached to the simulation. It was noted early in the simulation testing that flying a sweep of speeds was beneficial, as a wider range of the drag polar was flown. This result was included in much of the flight test planning.

After adequate data had been taken, the data was analyzed without adding simulated sensor noise. The results in Figure 5.1 show that the equations of motion used in the data analysis functions properly calculate the coefficients being passed into the system.

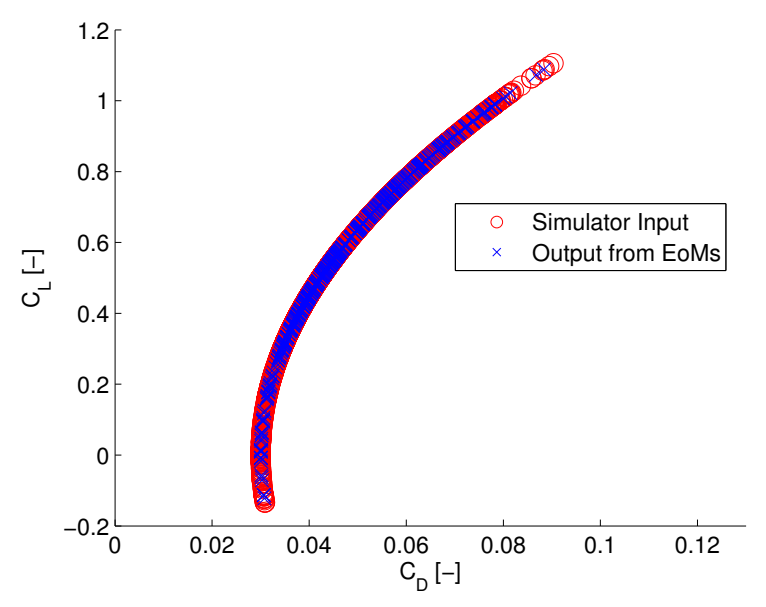

Figure 5.1: Data Analysis Verification (No Noise)

With this result, noise was added to the system to see how sensitive coefficient estimation was to noise in each sensor. This process was a balancing act between available sensor accuracy and the desired accuracy of the final solution. The final result guided sensor selection to those discussed in Section 6. To check if the final sensors chosen were acceptable, Gaussian noise was added to each state, with a mean of zero and a standard deviation equal to the 
RMS error listed in the manufacturer's data sheet for each sensor.

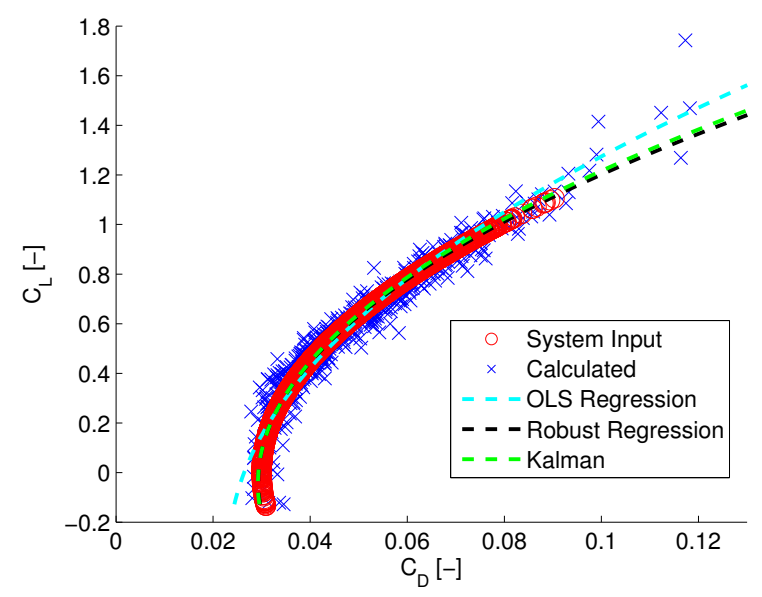

Figure 5.2: Drag Polar Prediction of Simulated Test Flight

For the particular simulated test flight shown in Figure 5.2, the estimated drag polar coefficients are shown in Table 5.1.

Table 5.1: Nonlinear Model Results

\begin{tabular}{cccc}
\hline \hline & $C_{D_{0}}$ & $C_{1}$ & $C_{2}$ \\
\hline System Inputs & 0.0493 & 0 & 0.03 \\
OLS Estimate & 0.0300 & 0.0196 & 0.0264 \\
Robust LS Estimate & 0.0461 & 0.0034 & 0.0294 \\
Kalman Estimate & 0.0446 & 0.0039 & 0.0293 \\
\hline
\end{tabular}

The results of this simulated flight test showed that the measurement system outlined in Section 6 predicted the simulated drag polar with a reasonable error. It also demonstrates the necessity of the heteroskedasticity correction, as the OLS regression has a $65 \%$ error on $K_{2}$ and a $13 \%$ error on $C_{D_{0}}$, while the robust regression has a $7 \%$ error on $K_{2}$ and a $2 \%$ error on $C_{D_{0}}$.

Since adding random noise results in stochastic error estimates, a Monte Carlo simulation was conducted to quantify expected accuracy values using the sensors selected in Section 6 . 
Error with a standard deviation equal to those reported by the manufacturer was added to clean simulated flight data, and the percent error in each coefficient was saved. This process was repeated 10,000 times, and the results are shown in Figures 5.3-5.5.

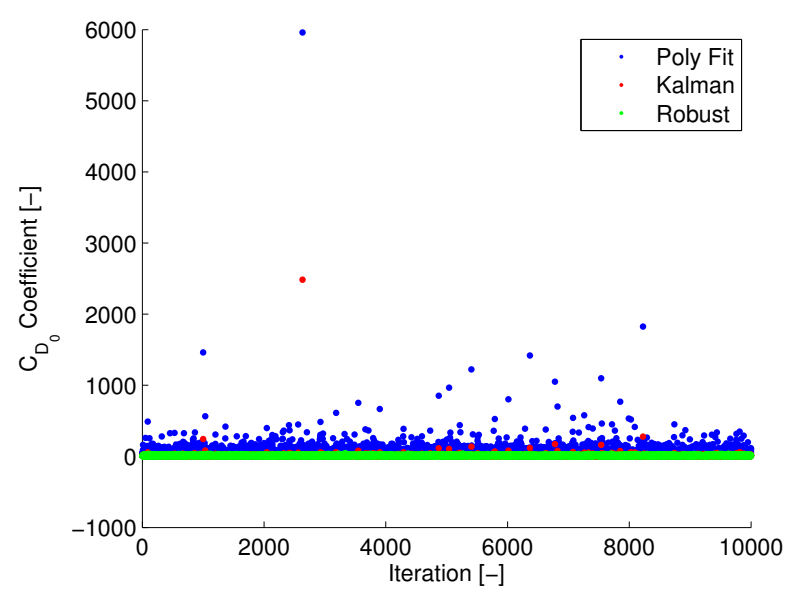

Figure 5.3: $C_{D_{0}}$ Monte Carlo Simulation

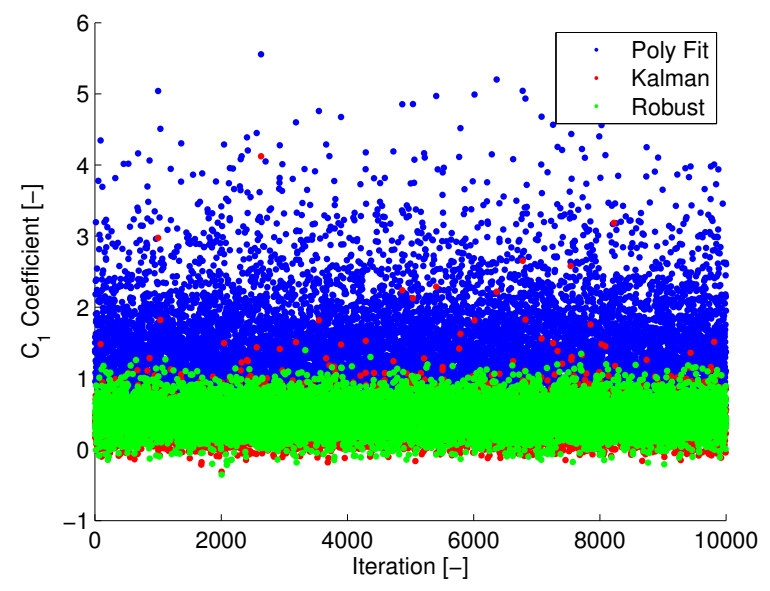

Figure 5.4: $C_{1}$ Monte Carlo Simulation 


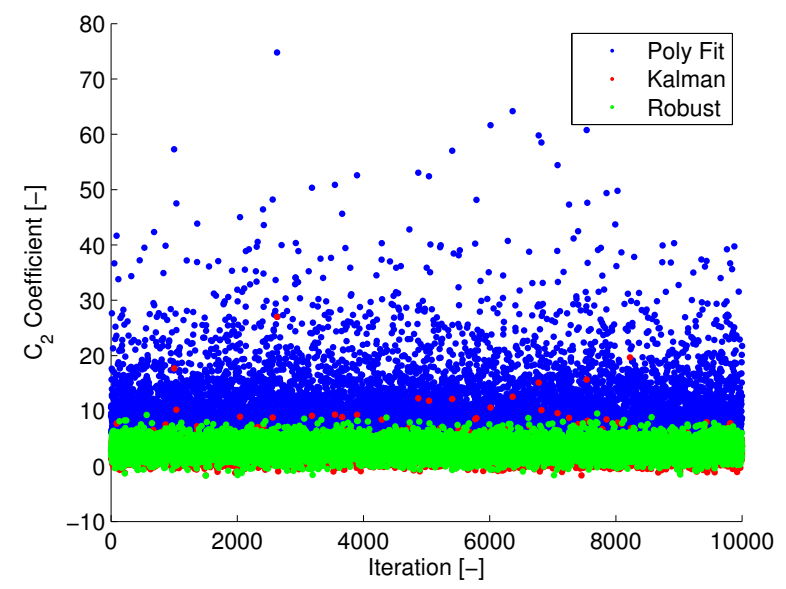

Figure 5.5: $C_{2}$ Monte Carlo Simulation

The results of the study, summarized in Table 5.2, show that the robustfit regression produced the most accurate and reliable results.

Table 5.2: Monte Carlo Results

\begin{tabular}{ccccccc}
\hline \hline & \multicolumn{3}{c}{ Mean Percent Error } & \multicolumn{3}{c}{ Percent Standard Error } \\
& $C_{D_{0}}$ & $C_{1}$ & $C_{2}$ & $C_{D_{0}}$ & $C_{1}$ & $C_{2}$ \\
\hline OLS & 53.8 & 1.5 & 10.6 & 81.7 & 0.6 & 6.0 \\
Robust LS & 12.2 & 0.5 & 3.2 & 6.3 & 0.2 & 1.5 \\
Kalman Filter & 12.1 & 0.4 & 2.5 & 26.0 & 0.2 & 1.3 \\
\hline
\end{tabular}




\subsection{Hardware}

One of the main goals of this research was to give the designer flexibility in choosing the appropriate sensors for a given flight test. To this end, hardware that is available in breakout boards was given preference, since it gives the aircraft designer more flexibility. The aircraft designer can utilize surface mount components if vehicle integration space is extremely limited, or can use the available breakout boards to make circuit-level integration easier if vehicle space is not a driving flight test concern.

\subsection{Flight Computer}

The flight computer chosen was an Arduino Due. This board has a 32-bit ARM processor, 54 digital I/O pins, 12 analog input pins, and 2 analog output pins. The main driver in the decision to use an Arduino-based platform was the vast support community, which allows quicker software development. The Arduino also offers a package that integrates well into most of the available airframes, and the stackable header pins allowed for easy integration with other boards. The Due in particular was chosen as it is (at the time of writing) the most advanced Arduino available. The main advantages it has over the comparable Arduino Mega is its increased clock speed (84 MHz for the Due ${ }^{[18]}$ vs $16 \mathrm{MHz}$ for the Arduino Mega ${ }^{[19]}$ ) and its 32-bit architecture (vs. 8-bit for the Arduino Mega).

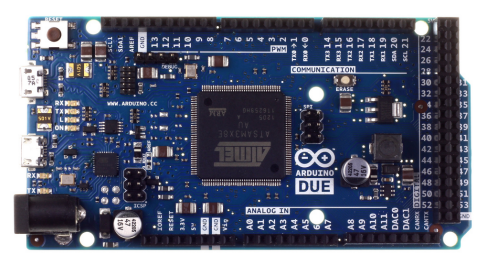

\section{Figure 6.1: Arduino Due Flight Computer}

The Arduino Due uses a 3.3V architecture instead of the usual Arduino architecture, 
which uses a 5V operating voltage. This was mainly beneficial, since most of the selected sensors used 3.3V as both supply and logic voltage. Logic level circuits, shown in Figure 6.2 , were used to translate to $5 \mathrm{~V}$ signals where required. The board is powered through the $3.5 \mathrm{~mm}$ barrel jack, using a 3-cell LiPo battery, with a nominal voltage of $11.1 \mathrm{~V}$.

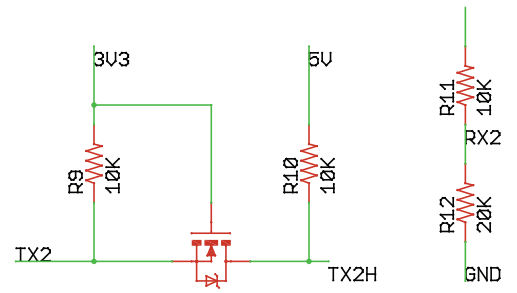

\section{Figure 6.2: Logic Level Converter Circuit}

\subsection{Accelerometer}

The accelerometer chosen for the data acquisition system was the ADXL-362 from Analog Devices. It has a noise error of $175 \mu \mathrm{G} / \sqrt{\mathrm{Hz}}$ and uses a $3.3 \mathrm{~V}$ digital SPI interface ${ }^{[20]}$. The accelerometer is in an LGA package and was surface mounted to the main PCB.

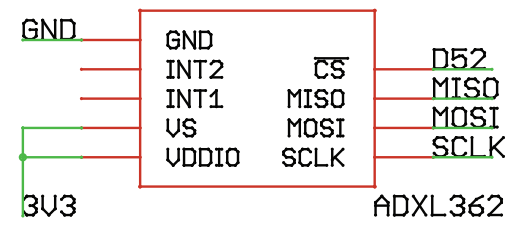

\section{Figure 6.3: ADXL-362 Schematic}

The accelerometer is calibrated in the field through a nonlinear least squares routine. For any given orientation, the accelerometer's reading can be expressed as

$$
\left[\begin{array}{ccc}
r_{x} & 0 & 0 \\
0 & r_{y} & 0 \\
0 & 0 & r_{z}
\end{array}\right]\left[\begin{array}{l}
m_{x} \\
m_{y} \\
m_{z}
\end{array}\right]+\left[\begin{array}{lll}
b_{x} & b_{y} & b_{z}
\end{array}\right]=\left[\begin{array}{lll}
\left(G_{x}-a_{x}\right) & \left(G_{y}-a_{y}\right) & \left(G_{z}-a_{z}\right)
\end{array}\right] .
$$

where the $r$ terms are the bit readings from the accelerometer for each axis, the $m$ terms are 
the slope of a linear fit for each axis, the $b$ terms are the zero offset of a linear fit for each axis, and the $a$ terms are the actual accelerations.

The slope and offset terms can be found using MATLAB's fmincon nonlinear constrained optimization function. The algorithm uses the fact that, while in a static orientation, the magnitude of the measured vector should be exactly $1 \mathrm{G}$. The optimization problem is then

$$
\underset{x}{\operatorname{minimize}} f(x)=\sqrt{(1 G-|(\vec{t})|)^{2}}
$$

where $\vec{t}$ is the right hand side of Equation 6.2.1, and the variable of interest $x$ is the vector of slopes and offsets in Equation 6.2.1. The slope terms in $x$ are constrained to be positive. To be a deterministic system of equations, at least six static orientations are required. To avoid the noise of a single reading, the problem was expanded to take 100 data points in six different static orientations, and Equation 6.2.1 was expanded to become a least squares problem.

The main benefit of this technique is that field calibration can be accomplished without needing precise knowledge of the orientation of gravity with respect to the sensor during the calibration routine.

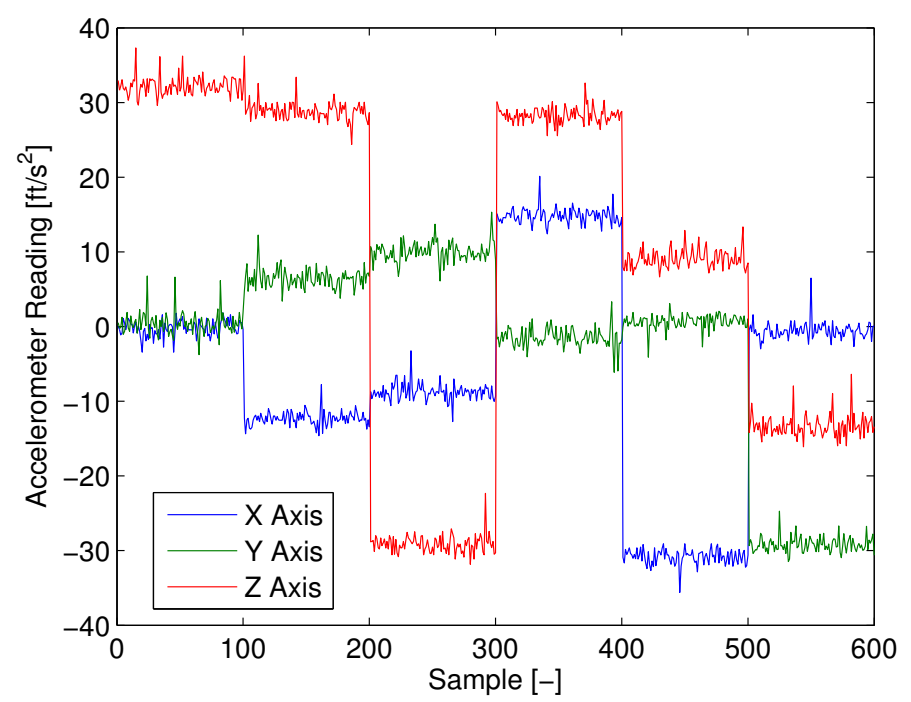

Figure 6.4: Calibration Results of ADXL-362 Accelerometer 
When tested, the results of the calibration were consistent between this algorithm and using known orientations. For error propagation, the noise during calibration was taken as the sensor's noise level.

\subsection{Vehicle Mass}

All test vehicles were weighed using a U-Line H-1650 counting scale. The scale has an accuracy of $0.001 \mathrm{lbs}$ and a maximum capacity of $30 \mathrm{lbs}$. The minimum capacity of the scale is 10 grams ${ }^{[21]}$.

\subsection{Magnetometers}

Two separate magnetometers were used for separate purposes. A Honeywell HMR-2300 3-D magnetometer, shown in Figure 6.5, is the main magnetometer. It is used when extremely accurate heading information is needed, or when GPS course is unavailable, such as during extremely slow or vertical flight.

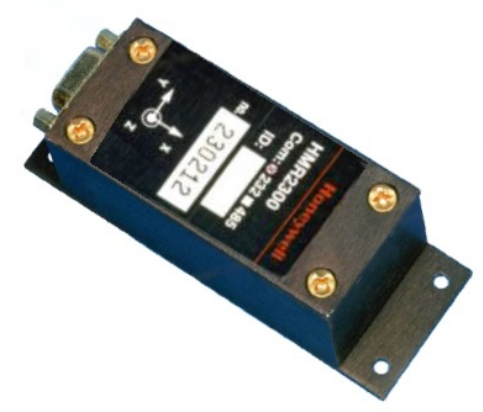

\section{Figure 6.5: Honeywell HMR-2300 3-D Magnetometer}

This magnetometer provides a RMS error of 0.1 milliGauss for all axes, using the 1 Gauss full-scale setting ${ }^{[22]}$.

The HMR-2300 can be supplied with power between $6 \mathrm{~V}$ and $15 \mathrm{~V}$, so the 3 -cell $11.1 \mathrm{~V}$ nominal LiPo battery that powers the Arduino also passes through to power the magnetometer. 
The HMR-2300 operates using an RS-232 serial interface. To properly interface with the Arduino Due, which uses 3.3V TTL logic levels, a Max-3232 IC was used. This IC, when combined with charge pump capacitors, translates TTL levels between 3V and 5.5V to RS-232 logic levels of $\pm 6 \mathrm{~V}$.
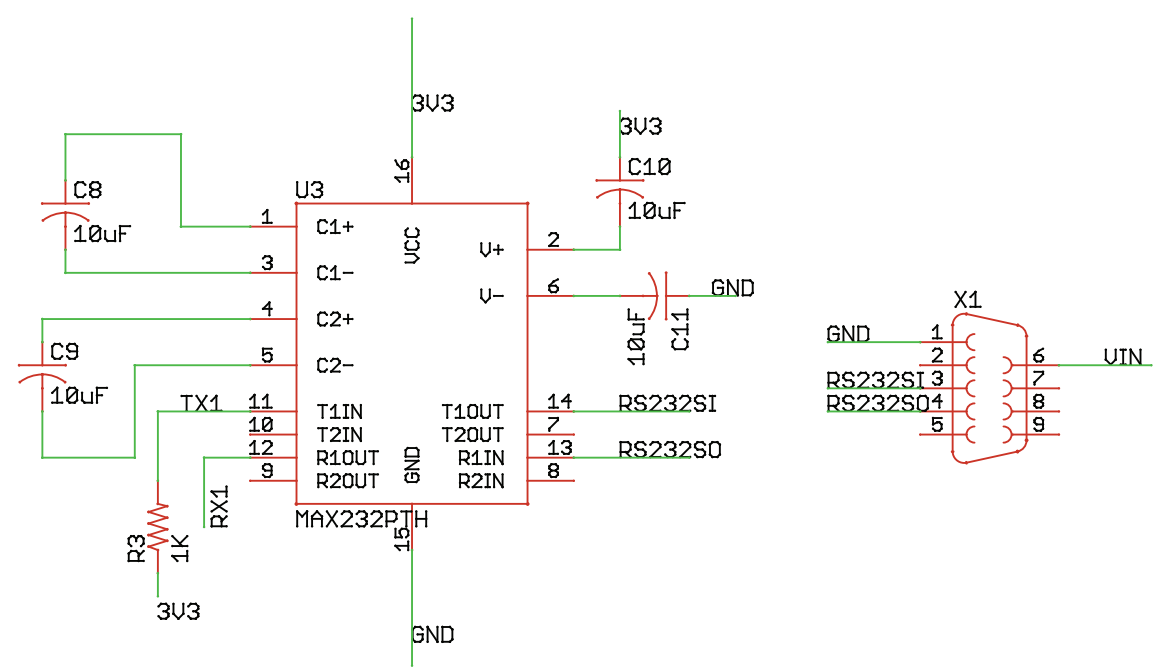

\section{Figure 6.6: HMR-2300 Logic Level Circuit}

The second magnetometer is a Honeywell HMC-5883L, which comes in an LCC package that was surface mounted to the main sensor board. It was added to the system for two main reasons: it is much smaller for applications where size is critical, and it is much less expensive for testing with unproven vehicles. It communicates with the Arduino using an $\mathrm{I}^{2} \mathrm{C}$ interface and uses a 3.3V operating voltage ${ }^{[23]}$. The HMC-5883L has an accuracy of 2 milliGauss on each axis. 


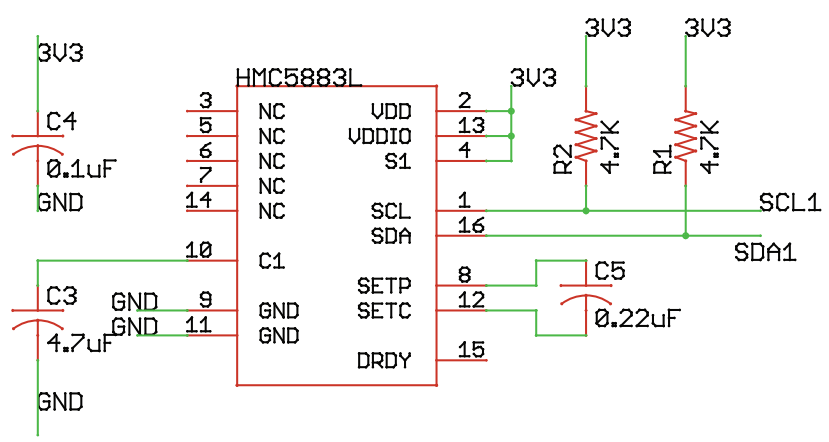

Figure 6.7: HMC-5883L Schematic

Both magnetometers were calibrated for both soft-iron and hard-iron effects ${ }^{[24]}$. To do this, data was acquired for 10 seconds with the magnetometer being swept through all directions. An ellipsoid was fit to the data using a ordinary least squares method available from the MATLAB file exchange ${ }^{[25]}$.

The least squares fit estimates the center and radius of each axis. The center values for each axis was subtracted from the readings to remove hard-iron effects. Each $i$-th axis is then scaled by $\frac{1}{R_{i}}$ to reshape the ellipse into a circle, which removes soft-iron effects.

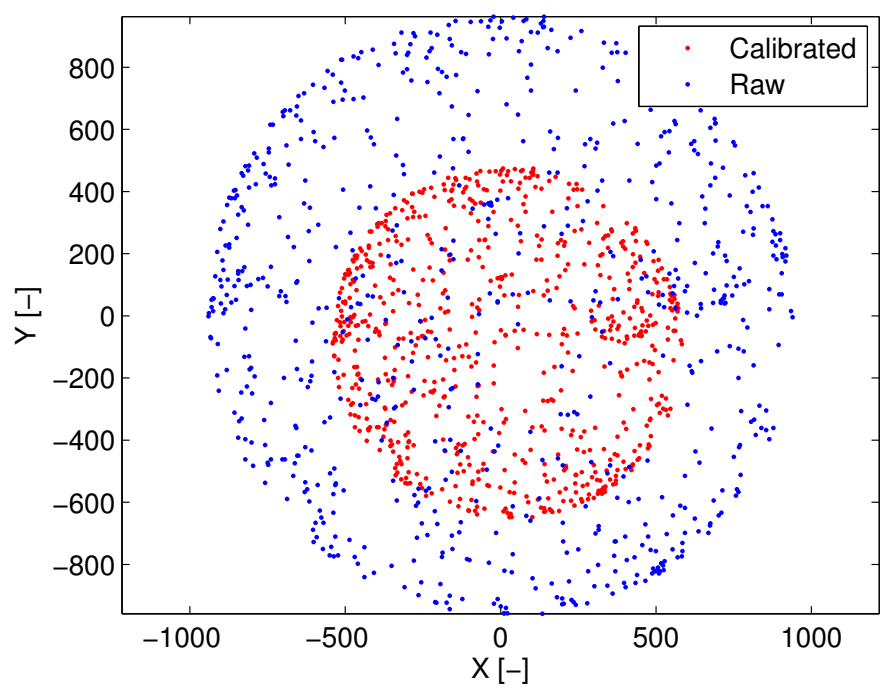

Figure 6.8: Soft- and Hard-iron Calibration for HMC-5883L 
The surface-mounted HMC-5883L was assumed to be aligned with the surface-mounted accelerometer. Since the two magnetometers both measured the North vector, a rotation matrix that describes the difference in alignment between the two sensors can be calculated by

$$
\vec{N}_{H M C 5883}=\bar{R}_{b}^{M_{1}} \vec{N}_{H M R 2300} .
$$

The rotation matrix $\bar{R}_{b}^{M_{1}}$ can then be used to align the HMR-2300's coordinate system with the body mounted accelerometers, using

$$
\vec{N}_{b}=\bar{R}_{b}^{M_{1}} \vec{N}_{H M R 2300}
$$

\subsection{Gyroscope}

A three-axis gyroscope was also included in the system. The gyroscope chosen was the Invensense ITG-3200, which comes in a QFN package. This gyroscope has a total error of $0.38^{\circ} / \mathrm{s}^{-r m s}{ }^{[26]}$, and uses a digital $\mathrm{I}^{2} \mathrm{C}$ interface on a $3.3 \mathrm{~V}$ operating voltage. This gyroscope has a full-scale span of $\pm 2000^{\circ} / \mathrm{s}$.

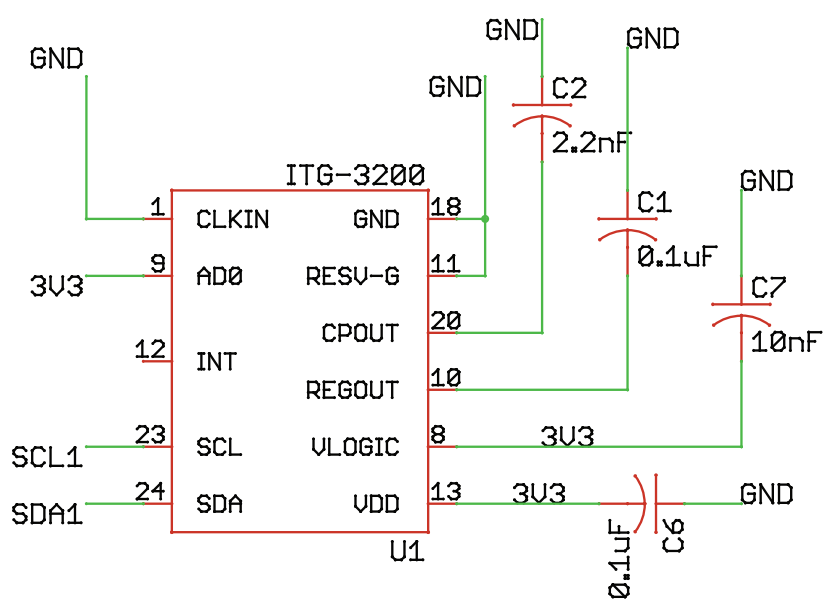

\section{Figure 6.9: ITG-3200 Eagle Schematic}

The gyroscope was calibrated in the same manner as the accelerometer. The device was placed in six orientations on a turn table which rotated at a constant $33 \frac{1}{3}$ RPM. Slope and 
offset values for each axis were calculated using fmincon. Before each flight test, the offset values were re-calculated by taking 10 seconds of static readings.

\subsection{Air Data System}

A five-hole probe was chosen to measure aerodynamic angles as they do not contain moving parts and can provide very accurate, repeatable data. The five-hole probe selected was the Aeroprobe Air Data probe. It is 6 inches long, has a diameter of $1 / 8$ inch, and uses a 0.25" hexagonal section as its mounting section. The probe comes factory calibrated from angles to pressure readings, and was calibrated at an airspeed of $70 \mathrm{ft} / \mathrm{s}$.

The probe was extended roughly one chord length in front of the leading edge of the wing by a 0.25 " carbon fiber tube and an aluminum adapter which connected to the probe using set screws.
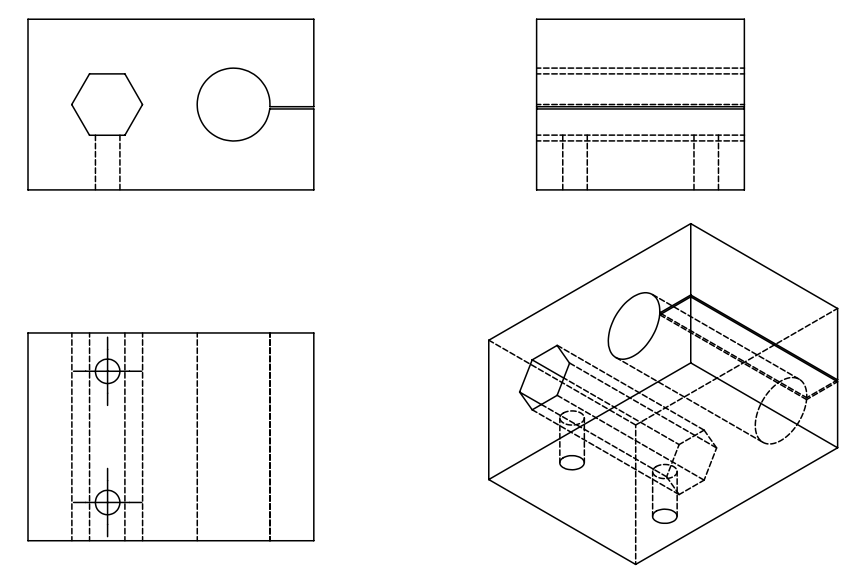

\section{Figure 6.10: Five-Hole Probe Adapter}

The adapter was manufactured to be square to itself and to have the reference flat of the probe's hexagonal section be parallel to one side of the adapter. An accelerometer was then glued to a side of the adapter, and the accelerometer was calibrated to the block using a level surface and the flat sides of the adapter. Before each flight test, three static readings 
are taken of the adapter's accelerometer and the main body-mounted accelerometer. Since the probe's orientation with respect to the adapter's accelerometer is known, this allows the wind angles measured by the probe to be calculated with respect to the body axes, and gives an accurate alignment of the wind reference frame to the body reference frame. The process is similar to how two magnetometers are aligned, using Equation 6.4.1.

Each pair of lines of the five-hole air data probe is connected to an All Sensors digital differential pressure sensor with a full scale range of \pm 5 in $-\mathrm{H}_{2} \mathrm{O}^{[27]}$. The static port of the five-hole probe was connected to an All Sensors BARO-DO digital barometric pressure sensor,which has a range of 600 to $1100 \mathrm{mBar}{ }^{[28]}$. The barometric pressure sensor comes in the same package and uses the same communication protocol as the differential pressure sensors.

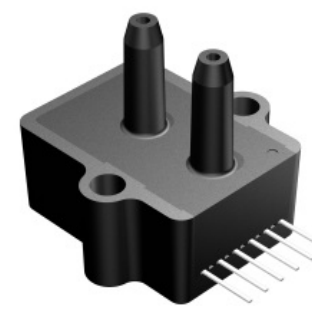

\section{Figure 6.11: All Sensors 5-INCH-D-DO Pressure Sensor}

The differential pressure sensors have a total error band of $0.25 \% \mathrm{FSO}$, and the barometric pressure sensor has a nominal error of $1 \mathrm{mBar}$. They use a UART serial interface that operates on a $5 \mathrm{~V}$ logic level, so the logic levels were converted to the $3.3 \mathrm{~V}$ levels of the Arduino Due. The serial interface includes addressable read commands, which allows multiple devices on a single data bus, and ensures all devices record pressure at the same time. The sensor can output both a 14-bit pressure reading and a 12-bit temperature reading, which the device uses to correct its pressure measurement.

A digital temperature sensor was combined with the barometric pressure sensor to estimate 
the air density, which allowed air speed to be calculated.

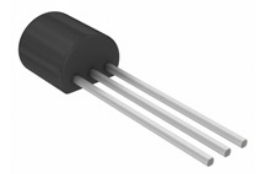

\section{Figure 6.12: Dallas Semiconductors' DS18B20 Digital Temperature Sensors}

The DS18B20 from Dallas Semiconductors was chosen for its relatively simple One-Wire interface. The device can be powered with the communication line and has a $\pm 0.5^{\circ} \mathrm{C}$ nominal $\operatorname{accuracy}^{[29]}$.

\subsection{GPS Receiver}

A uBlox LEA-6T GPS receiver was included in the data acquisition system. This model was selected for its ability to output raw timing data, which can be used to get an extremely accurate inertial velocity estimate ${ }^{[32]}$. The receiver itself was integrated onto a breakout board sold by CSG Shop, which has UART, USB, and $\mathrm{I}^{2} \mathrm{C}$ interface options.

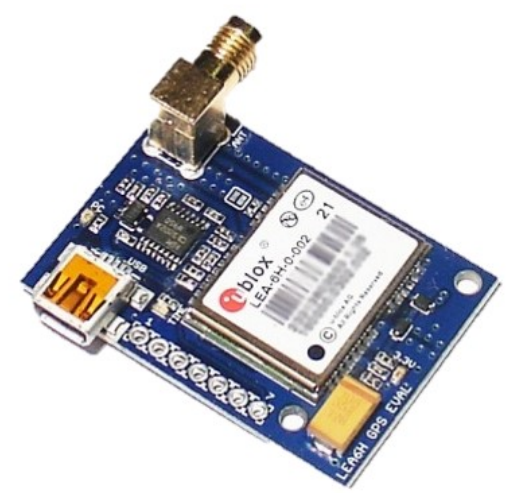

Figure 6.13: CGS Shop Board for uBlox LEA-6T 


\subsection{Data Acquisition System Integration}

The sensors were packaged into a main shield for the Arduino. This shield plugs directly into the Arduino, eliminating the need to disconnect and reconnect wiring. Header pins capable of reading commanded PWM signals to servos were also added on the main board. Future work could include stability derivative estimation, and the header pins provide PWM measurement, which can map to servo angles, if it is assumed the servo is not stalled. The servo signal breakout pins also allowed the data to be easily split into sections with and without commanded throttle for drag polar estimation without thrust. All data was saved to a microSD card attached to the main sensor board. Data was saved in binary format for both increased speed and file size reductions. Once on the ground, the data is converted to meaningful values using a custom MATLAB data parser.

A second board was developed to integrate the air data system with the main sensor board. This pressure board can be located near a wing tip and provides expandability should additional sensors be desired in the future. It also interfaces with the temperature sensor, which is located in the air flow. 


\subsection{Results}

The system was built and tested to prove functionality. After functionality testing was complete, the system was integrated into a 0.60-size Piper Cub R/C aircraft. The aircraft weighed $10.0 \mathrm{lb}$, had a wing area of $6.85 \mathrm{ft}^{2}$, and a wing span of $7 \mathrm{ft}$.
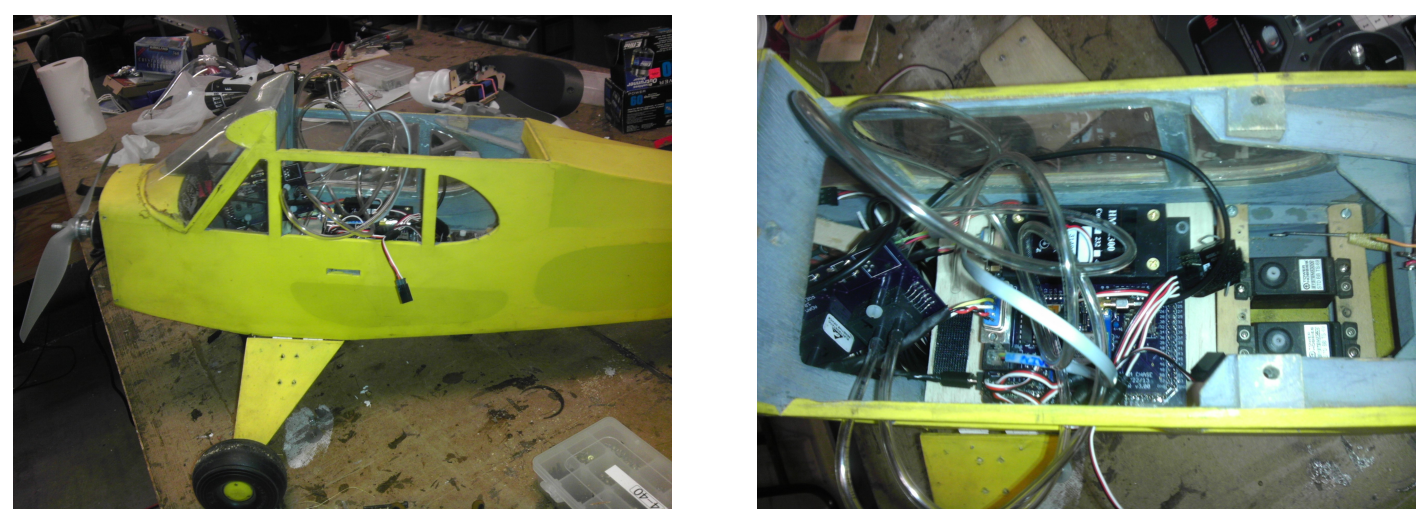

Figure 7.1: System Integration into 0.60-size R/C Piper Cub

Flight testing was completed at Cal Poly's Education Flight Range. Each flight test included multiple stalls and high speed dives, so that as much of the flight envelope was covered as possible. More information on specific flight test procedures is available in Appendices A and D.

\subsection{Drag Polar}

A drag polar captured from flight data is shown in 7.2. The red lines in figure 7.2 are 2-D error bars calculated according to Section 4.1. 


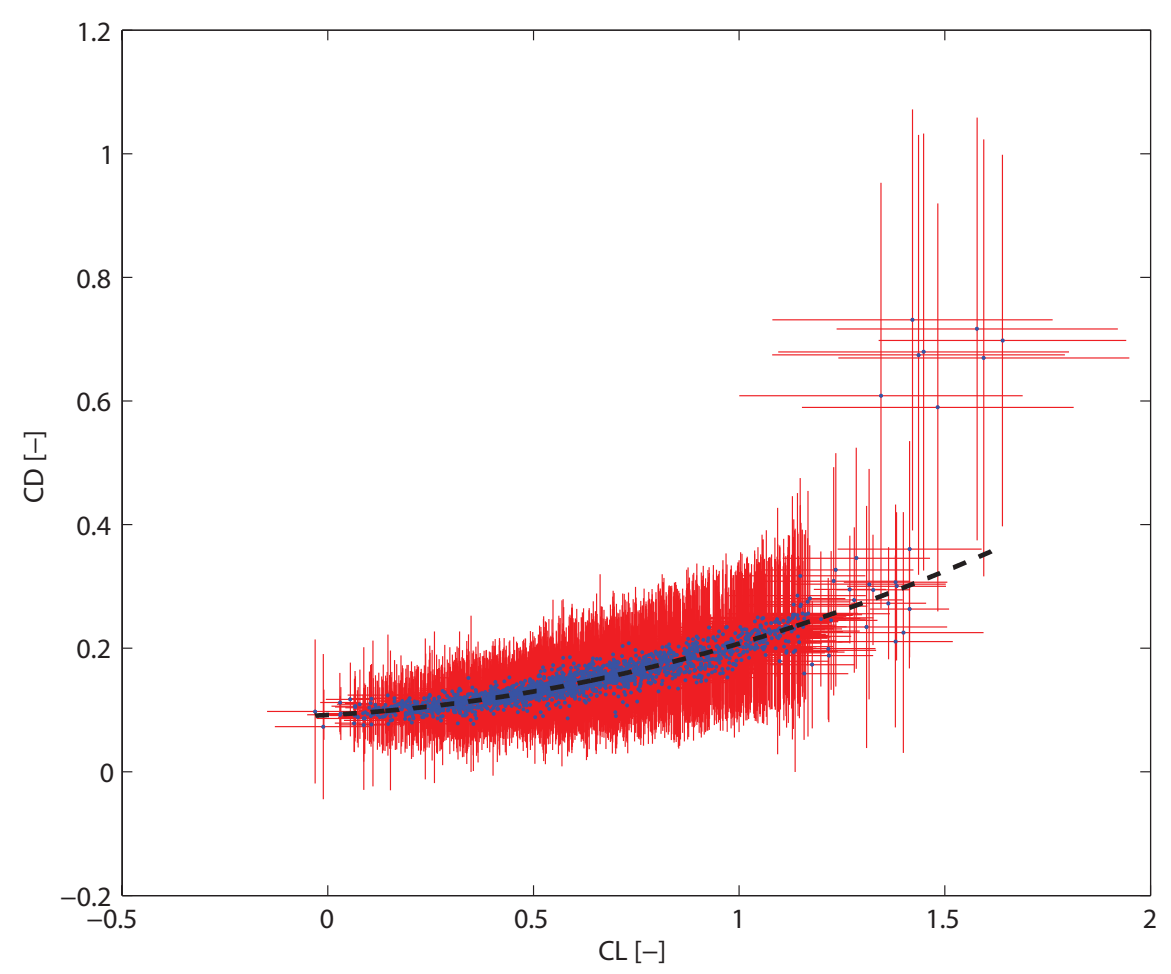

Figure 7.2: Drag Polar from Flight Test

For this particular flight, the drag polar regression coefficients are shown in Equation 7.1.1.

$$
C_{D}=0.079243 C_{L}^{2}+0.036290 C_{L}+0.092005
$$

Sections of particular interest in the drag polar are discussed below.

\subsection{1 $C_{2}$ Coefficient}

The wing used on this flight test had a chord of 11.75 inches and a span of 7 feet, which corresponds to an aspect ratio of 7.15 . Recalling that the $C_{2}$ regression coefficient is

$$
C_{2}=K_{1}+K_{2}
$$

where $K_{1}$ is the profile drag term from the airfoil, and $K_{2}$ is the inviscid correction for a finite wing, as shown in Equation 7.1.3. 


$$
K_{2}=\frac{1}{\pi e A R}
$$

Rearranging Equation 7.1.3 for the ellipticity value $e$ gives

$$
e=\frac{1}{\pi A R\left(C_{2}-K_{1}\right)}
$$

The $K_{1}$ term can be found using Equation 7.1.5,

$$
K_{1}=\frac{C_{1}}{-2 C_{L_{M I N}}}
$$

where $C_{L_{M I N}}$ is calculated from XFOIL and is 0.26 for the Clark-Y airfoil used. For this flight test, $e=0.30$. A different method of estimating the ellipticity value is also discussed in Section 7.3.

\subsubsection{Drag Break}

One of the benefits of the heteroskedastically-robust least squares regression is it's ability to remove outliers from the regression model. This is of substantial benefit when looking at break drag. Break drag is the non-parabolic drag rise that occurs at and past stall. Break drag is evident in Figure 7.2, around $C_{L}=1.5$. If the regression model had used an ordinary least squares, the regression scheme would have equally weighted these points and led to an artificially steep parabola. Instead, it's evident that these points past $C_{L_{B R E A K}}$ are the only points for which their error bars do not overlap the regression curve.

\subsubsection{Error Estimation}

One of the key lessons learned from the simulator was that the error in lift and drag prediction is heteroskedastic, and increases as the lift coefficient increases. A plot of the drag polar residuals is shown in 7.2 . 


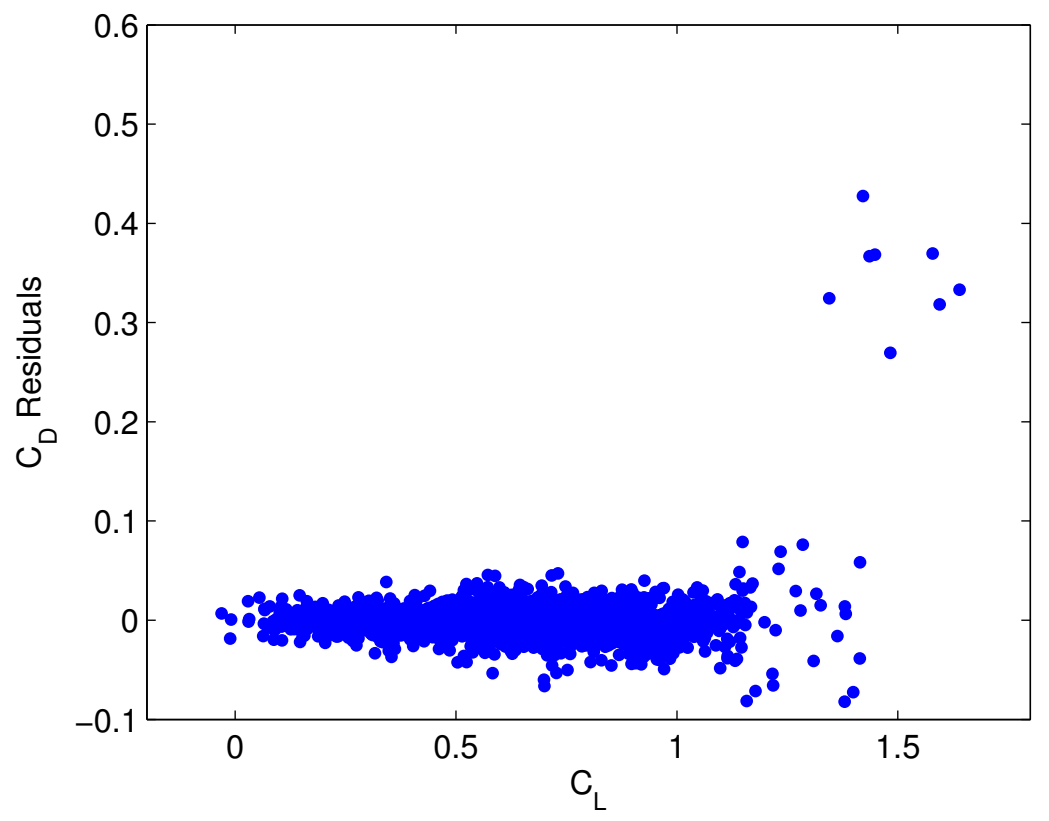

Figure 7.3: Drag Polar Residuals from Flight Test

The scatter of the data matches the trend seen in the simulator. Namely, as $C_{L}$ goes towards zero, the scatter decreases. This is a direct result of the heteroskedasticity. Since the error is a function of the state, as $C_{L}$ goes to zero, that contribution of error drops out.

\subsection{Lift Curve}

A plot of lift coefficient versus angle of attack captured from flight data is shown in 7.4. Also overlayed is airfoil sectional lift characteristics, taken from XFOIL. 


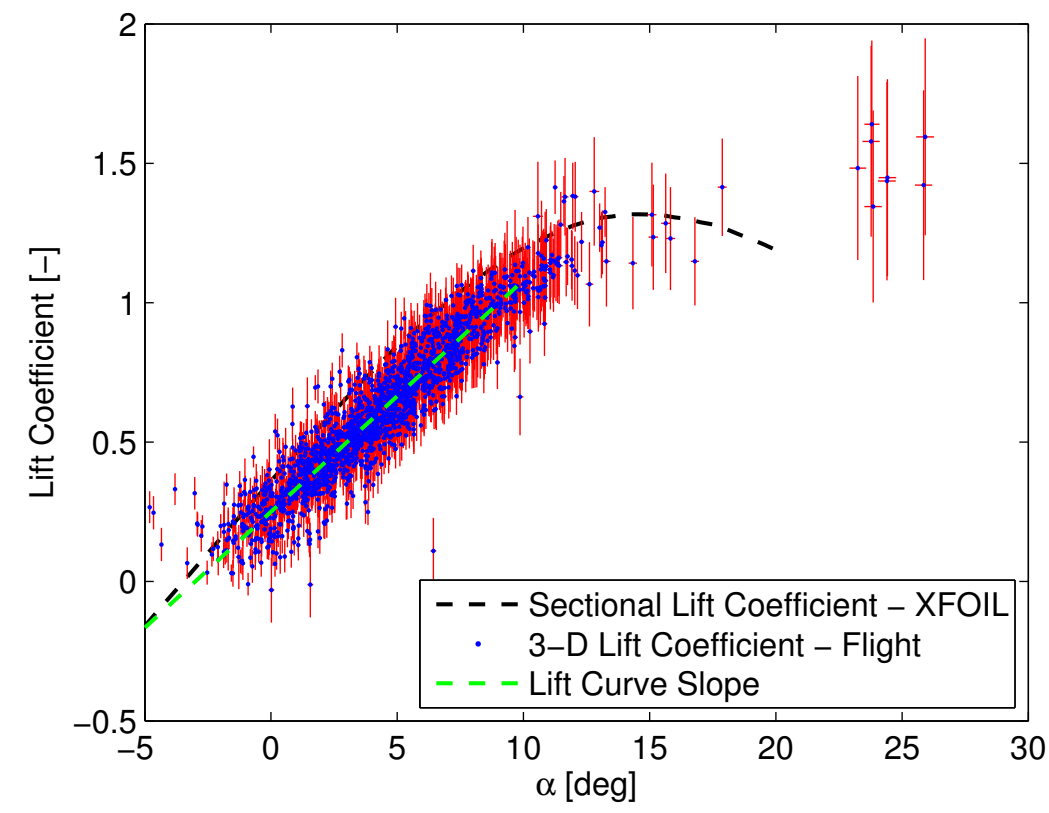

Figure 7.4: Lift Curve from Flight Test

Sections of particular interest in the lift curve slope are discussed below.

\subsection{1 $C_{L_{M A X}}$ and Stall}

Due to the heteroskedastic nature of the error, the error in $C_{L}$ increases as $C_{L}$ increases, which makes it particularly difficult to estimate $C_{L_{M A X}}$ with little error. However, the approximate maximum lift coefficient in Figure 7.4 matches well with the estimate of the maximum lift coefficient found using XFOIL. The stall angle of attack appears to be accurate for the set of semi-continuous data. The group of data centered around $\alpha=25^{\circ}$ is disconnected from the main group of data, as shown Figure 7.5. 


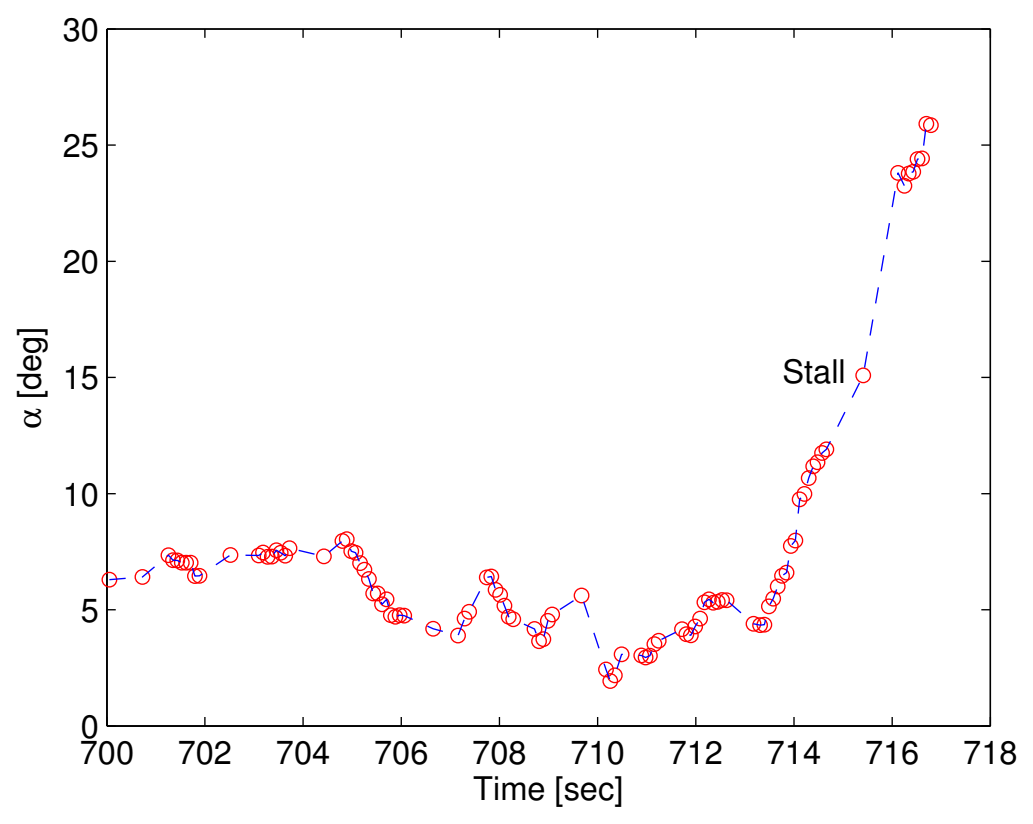

Figure 7.5: Angle of Attack History from Flight Test

The data point at 15 degrees angle of attack corresponds to the stall angle of attack as estimated in XFOIL. After that, a sudden increase in angle of attack occurs as the vehicle stalls. The stall causes the vehicle to rapidly lose altitude, which increases the angle of attack as it falls. This effect causes the discontinuity and makes the original stall angle of attack, which matches that calculated by XFOIL, more accurate.

\subsubsection{Lift Curve Slope}

The lift curve slope $\left(C_{l_{\alpha}}\right)$ of a thin airfoil is $2 \pi C_{L}$ per radian according to thin airfoil theory. This decreases due to finite wing corrections, and is a function of wing aspect ratio and ellipticity, as shown in Equation 7.2.1

$$
C_{L_{\alpha}}=\frac{C_{l_{\alpha}}}{1+\frac{C_{l_{\alpha}}}{\pi e A R}}
$$

where $C_{L_{\alpha}}$ is the $3-\mathrm{D}$ lift curve slope for the wing. Using the ellipticity value found earlier of $e=0.30$, this gives a $3-\mathrm{D}$ lift curve slope of $C_{L_{\alpha}}=3.24$. The slope of the linear regression 
line in Figure 7.4 is $C_{L_{\alpha}}=4.76$, which corresponds to a $32 \%$ error. Again, a different method of estimating this value is presented in Section 7.3.

\subsubsection{Zero Lift Angle of Attack}

The 3-D wing corrections only affect the wing when it is producing lift. Therefore, the zero lift angle of attack should stay constant between the 2-D airfoil data analyzed in XFOIL and the 3-D wing data collected in flight. The zero lift angle of attack from XFOIL was -3.0 degrees, and the zero lift angle of attack from flight test was -3.5 degrees.

\subsection{System Repeatability}

The system was validated for both repeatability and accuracy. For repeatability, three flight tests were conducted in a clean configuration, and the data was analyzed to verify consistency. A plot of the drag polars from the three flight tests is shown in Figure 7.6. 


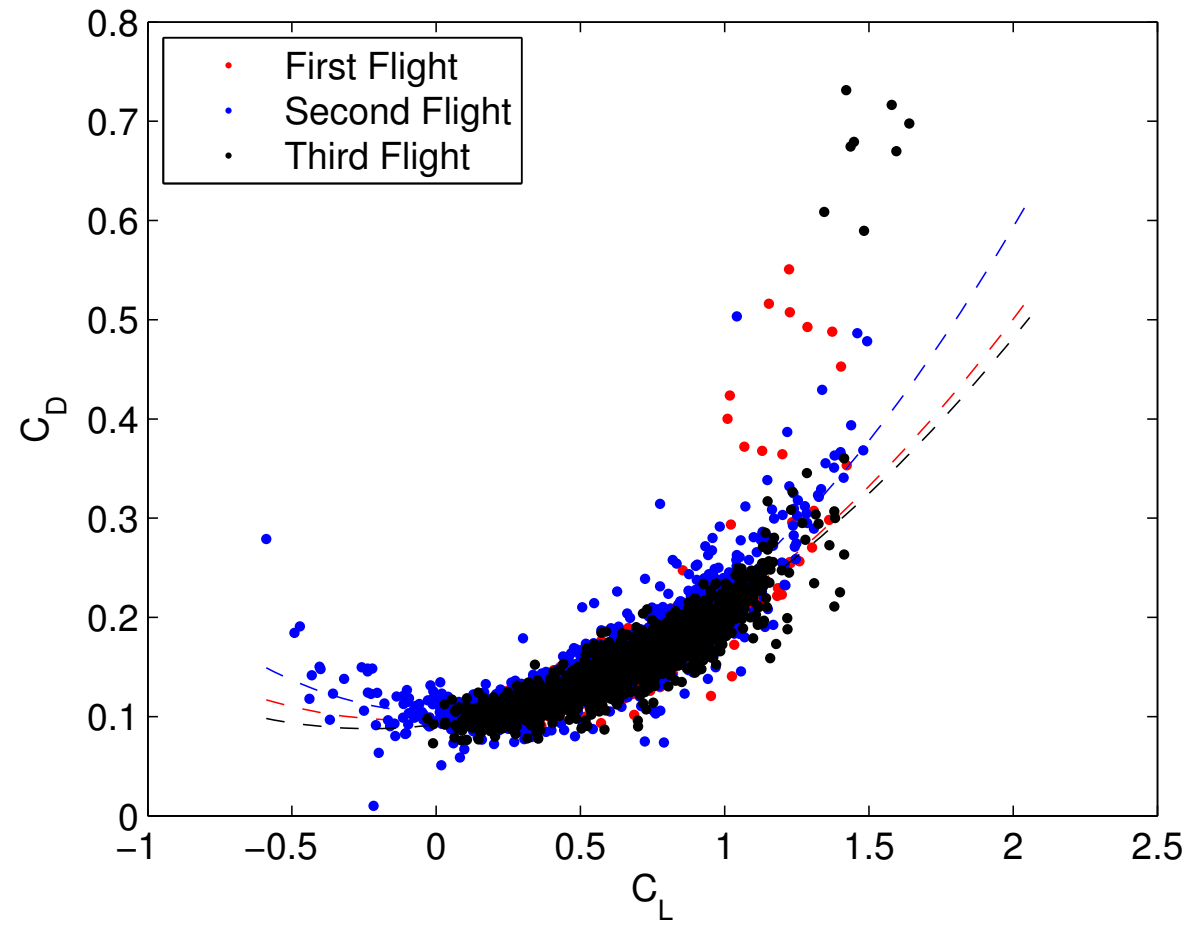

Figure 7.6: Drag Polar Repeatability Testing

The estimates of the regression model coefficients for three flight tests are shown in Tables 7.1-7.3. 
Table 7.1: Clean Drag Polar Repeatability Testing : $C_{D_{0}}$ Regression Coefficient

\begin{tabular}{|c|c|c|}
\hline Flight Number & $C_{D_{0}}$ Estimate & 95\% Confidence Interval \\
\hline 1 & 0.097872 & $0.1170 / 0.0788$ \\
\hline 2 & 0.103945 & $0.1095 / 0.0984$ \\
\hline 3 & 0.092005 & $0.0981 / 0.0859$ \\
\hline Combined Flights & 0.100037 & $0.1038 / 0.0963$ \\
\hline
\end{tabular}

Table 7.2: Clean Drag Polar Repeatability Testing : $C_{1}$ Regression Coefficient

\begin{tabular}{|c|c|c|}
\hline Flight Number & $C_{1}$ Estimate & 95\% Confidence Interval \\
\hline 1 & 0.020783 & $0.0497 /-0.0082$ \\
\hline 2 & -0.002644 & $0.0039 /-0.0092$ \\
\hline 3 & 0.036290 & $0.0446 / 0.0280$ \\
\hline Combined Flights & 0.011454 & $0.0163 / 0.0066$ \\
\hline
\end{tabular}

Table 7.3: Clean Drag Polar Repeatability Testing : $C_{2}$ Regression Coefficient

\begin{tabular}{ccc}
\hline \hline Flight Number & $C_{2}$ Estimate & $95 \%$ Confidence Interval \\
\hline 1 & 0.090236 & $0.1009 / 0.0796$ \\
2 & 0.123529 & $0.1255 / 0.1215$ \\
3 & 0.079243 & $0.0819 / 0.0766$ \\
\hline \hline Combined Flights & 0.101002 & $0.1025 / 0.0995$ \\
\hline
\end{tabular}

The parasite drag coefficient was very repeatable for the system. Flights 1 and 3 showed good repeatability for the $C_{1}$ regression coefficient, but the second flight was an order of magnitude lower. The $C_{2}$ regression coefficient also showed good repeatability for flights 1 and 3 , but was roughly $25 \%$ different for flight 2 . This trend is not surprising, since the slope of a linear regression model is more difficult to estimate than the intercept. This is due to 
there being error in both $C_{L}$ and $C_{D}$, and estimating the slope requires multiplying the error in both terms, instead of only the error in $C_{D}$ for the intercept term.

A plot of the correlation between the regression model and flight test data is shown in Figure 7.7, and a plot of the residuals of the regression model is shown in Figure 7.8.

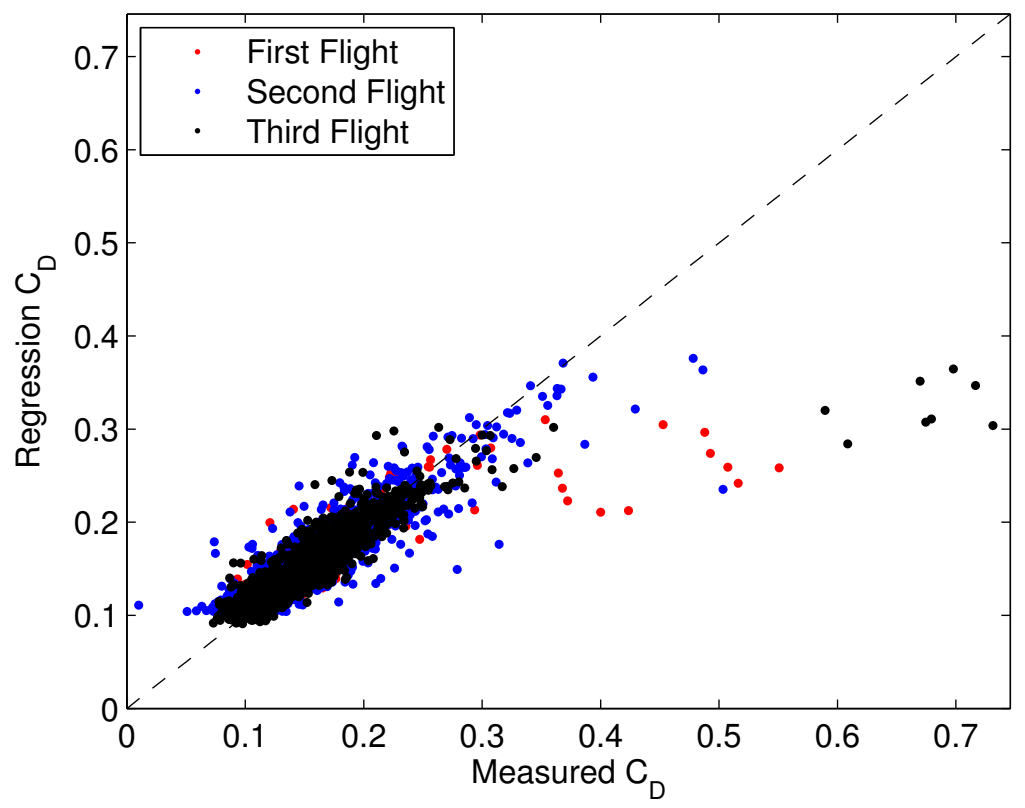

Figure 7.7: Correlation of Regression Model and Flight Data 


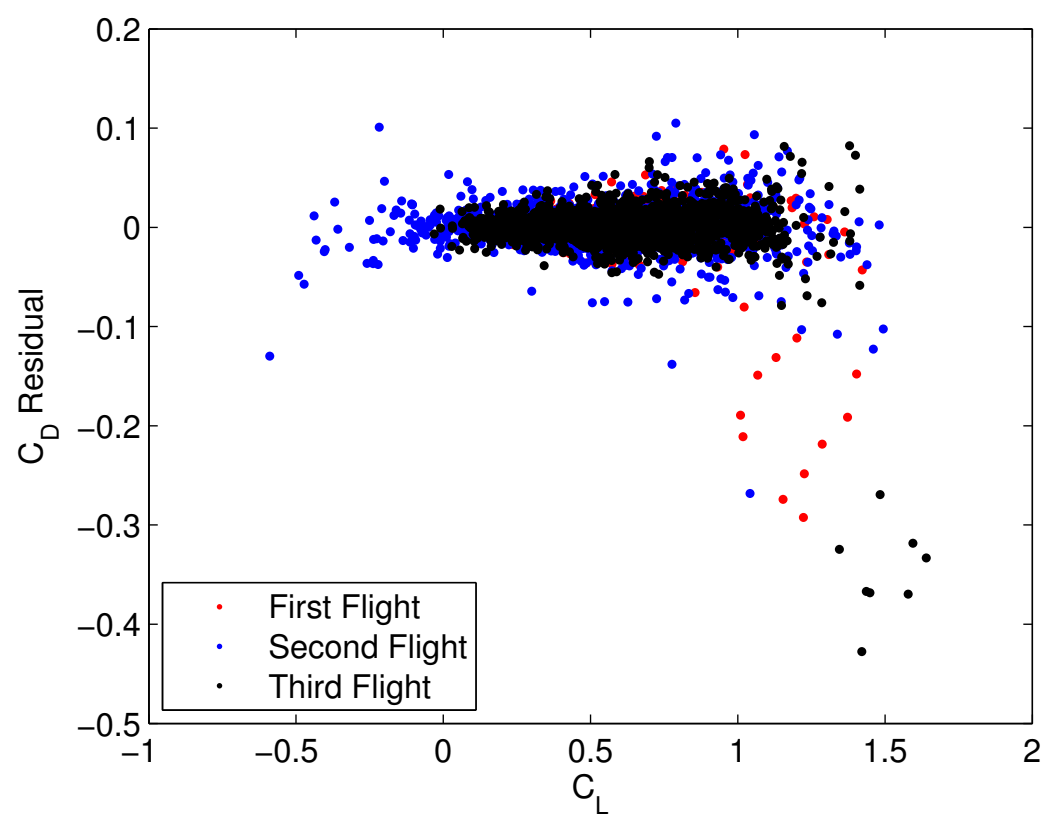

Figure 7.8: Residuals of Regression Model and Flight Data

The altered correlation and increased residuals past $C_{L_{B R E A K}}$ values was expected, and is a result of using a heteroskedastically-robust estimator. Also note the error increasing on either side of $C_{L}=0$, which compares well to the simulator data in Figure 4.1. The system shows similar repeatability for the aircraft's lift characteristics as it does for the drag polar regression. Flight data for three clean flights is shown in Figure 7.9. 


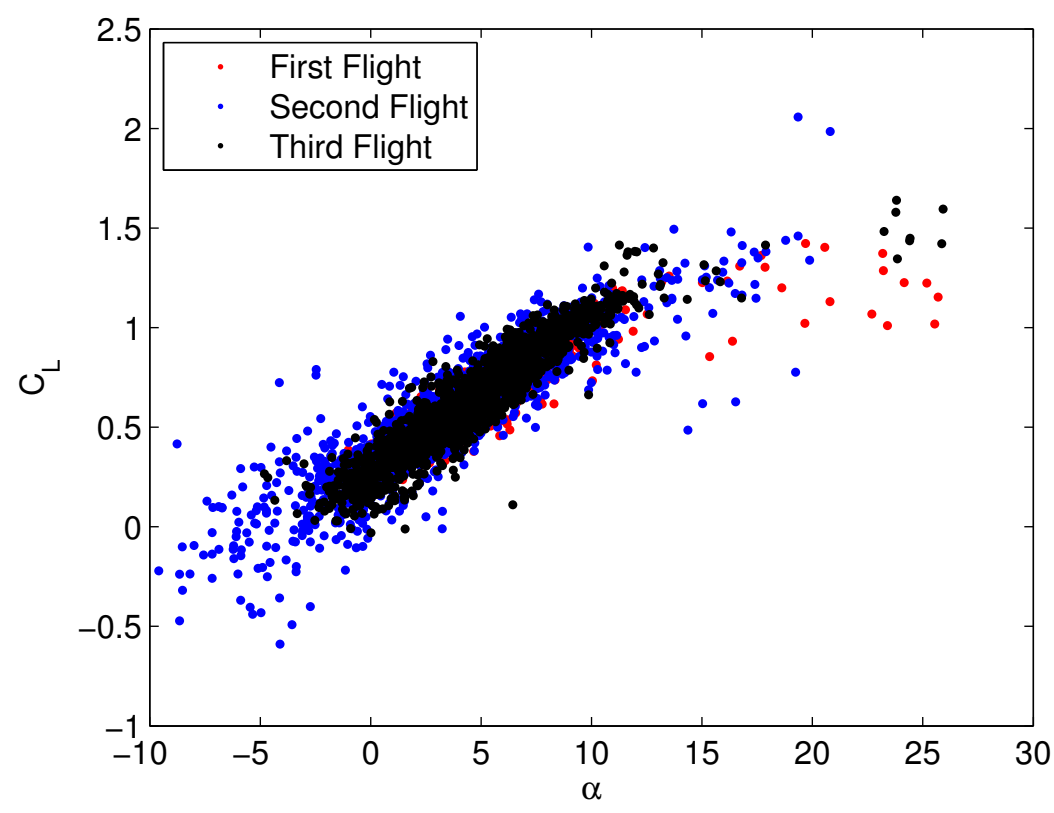

Figure 7.9: Lift Curve Repeatability Testing

Table 7.4 shows a summary of the zero lift angle of attack. The average zero lift angle of attack is in very good agreement with XFOIL's value of -3.0 degrees.

\begin{tabular}{cc}
\multicolumn{2}{c}{ Table 7.4: Lift Curve Model } \\
\hline \hline Flight Number & $\alpha_{0 L}$ Estimate \\
\hline 1 & $-2.2^{\circ}$ \\
2 & $-3.4^{\circ}$ \\
3 & $-3.5^{\circ}$ \\
\hline \hline Combined Flights & $-3.0^{\circ}$ \\
\hline
\end{tabular}

The scatter in the $C_{1}$ and $C_{2}$ regression coefficients makes estimation of the ellipticity value less accurate, as seen with the previously low estimate of $e=0.30$. However, the $C_{1}$ regression coefficient can be calculated directly using XFOIL, as shown in Figure 7.10. 


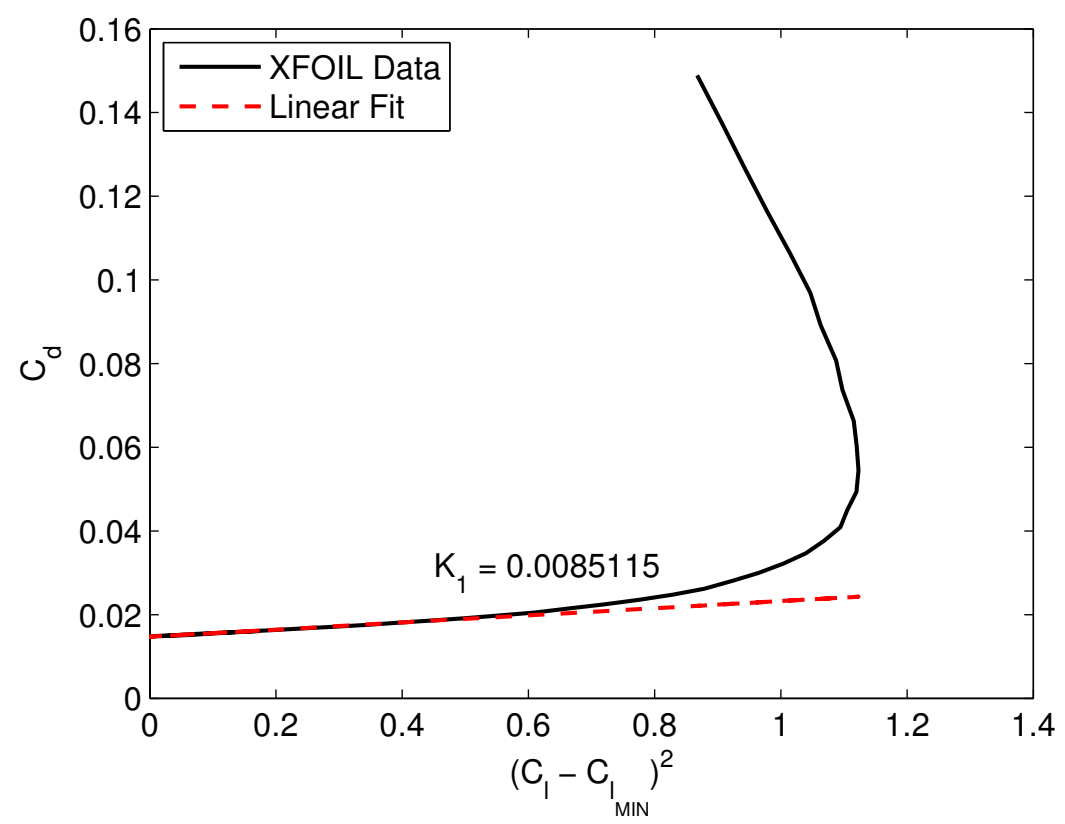

Figure 7.10: XFOIL-based $C_{1}$ Estimation

If that value is used, the estimate of the ellipticity value found from flight test data becomes more reasonable, which makes the lift curve slope match better to that estimated by lifting line theory. These results are summarized in Table 7.5.

Table 7.5: Ellipticity Value with $C_{1}$ From Flight and XFOIL

\begin{tabular}{ccccc}
\hline \hline Flight Number & \multicolumn{2}{c}{$C_{1}$} & \multicolumn{2}{c}{$e$ Estimate } \\
\hline & Flight & XFOIL & Flight & XFOIL \\
1 & 0.020783 & 0.0085115 & 0.34 & 0.54 \\
2 & -0.002644 & 0.0085115 & 0.38 & 0.39 \\
3 & 0.036290 & 0.0085115 & 0.30 & 0.63 \\
\hline \hline Combined Flights & 0.011454 & 0.36 & & 0.48 \\
\hline
\end{tabular}

The ellipticity values from Table 7.5 were used in Equation 7.2.1, and the results are shown in Table 7.6. 


\section{Table 7.6: Lift Curve Slope with $C_{1}$ From Flight and XFOIL}

\begin{tabular}{ccccccc}
\hline \hline Flight Number & \multicolumn{2}{c}{$C_{1}$} & \multicolumn{2}{c}{$C_{L_{\alpha}}$ Estimate } & \multicolumn{2}{c}{ PercentError } \\
\hline & Flight & XFOIL & Flight & XFOIL & Flight & XFOIL \\
1 & 0.020783 & 0.0085115 & 3.46 & 4.15 & 27 & 13 \\
2 & -0.002644 & 0.0085115 & 3.60 & 3.65 & 24 & 23 \\
3 & 0.036290 & 0.0085115 & 3.24 & 4.35 & 32 & 9 \\
\hline \hline Combined Flights & 0.011454 & 0.0085115 & 4.76 & 3.9738 & 25 & 16 \\
\hline
\end{tabular}

In the end, both this ellipticity estimate, and the one calculated according to the regression coefficient alone are poor estimates. There are many reasons why this is the case. First, the $C_{L_{M I N}}$ calculated from XFOIL is for the airfoil alone and does not account for angle of attack differences between the probe and the airfoil itself. It also does not account for the fuselage drag, or a twisted wing, or any dihedral in the wing, or any other 3-D effects. So the vehicle's $C_{L_{M I N}}$ could be drastically different than the airfoil's $C_{L_{M I N}}$. Without accurate XFOIL data for the whole aircraft, the system becomes indeterminate, and it's not possible to isolate the $K_{2}$ term from the $C_{2}$ regression coefficient.

\subsection{System Accuracy}

The accuracy of the system was validated by adding additional parasite drag of a known amount and measuring the difference in the vehicle's parasite drag coefficient. This was accomplished using a cone that was trailed behind the aircraft, shown in Figure 7.11. 

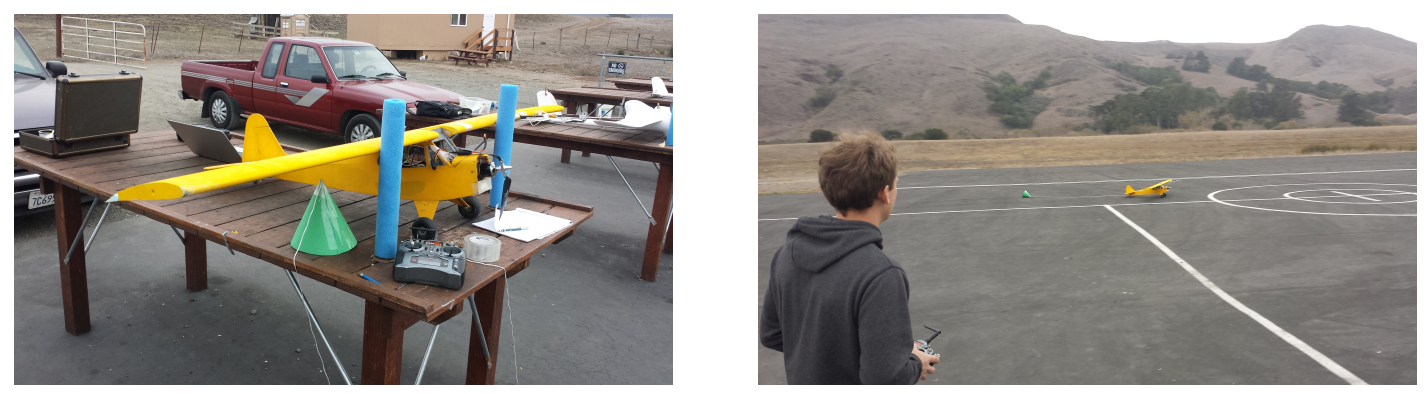

Figure 7.11: Parasite Drag Cone Integration

The drag coefficient of a cone, as a function of its half-vertex angle, is shown in 7.12.

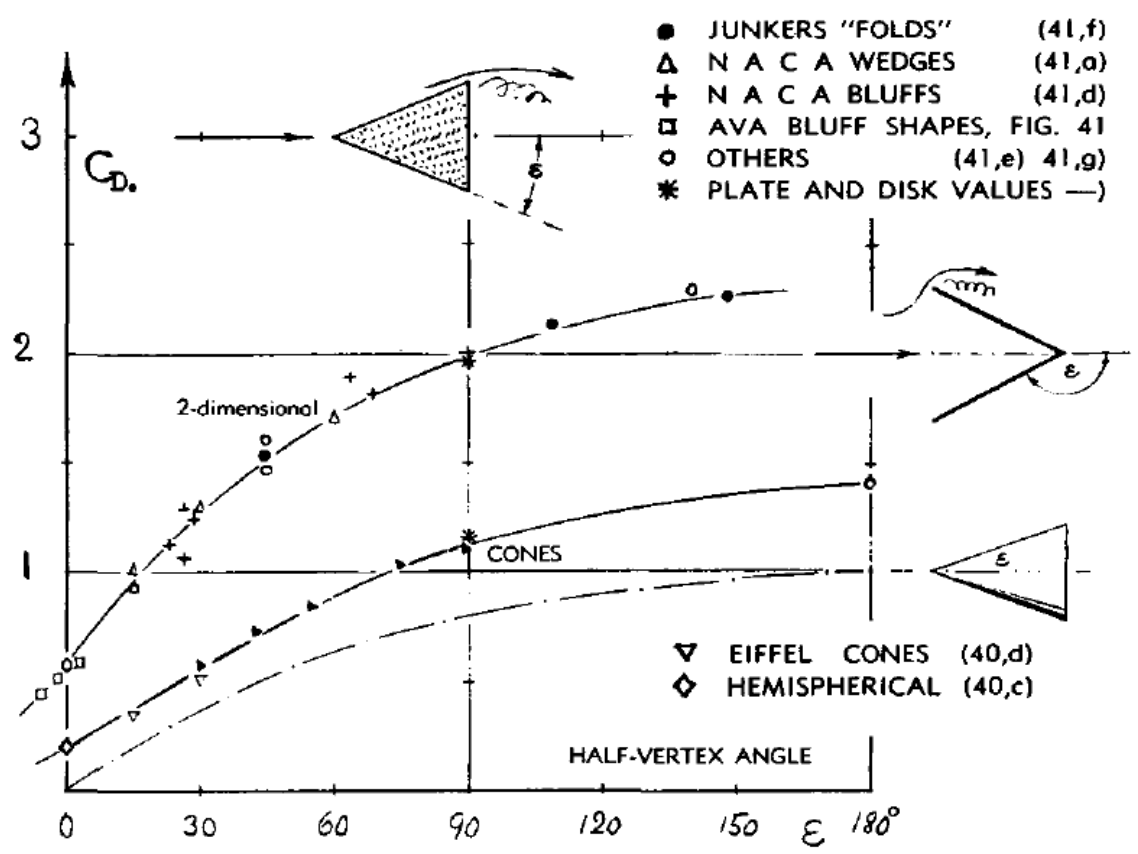

Figure 7.12: Cone Drag as a Function of Half Angle ${ }^{[4]}$

The cone was 8 inches tall and had a radius of 4.25 inches, which corresponds to a half-vertex angle of 28.0 degrees. Figure 7.12 was plot digitized, showing that a half-vertex angle of 28.0 degrees corresponded to a drag coefficient of between 0.50 and 0.56 , based on the spread of the source data. For system validation, the mean of this range $\left(C_{D}=0.53\right)$ will be used. When scaled by the reference area, this was equal to an additional 305 counts of 
drag.

The system was tested three times while trailing the cone. The results are shown in Tables 7.7-7.9. 
Table 7.7: Dirty Drag Polar Repeatability Testing : $C_{D_{0}}$ Regression Coefficient Flight Number $\quad C_{D_{0}}$ Estimate $95 \%$ Confidence Interval

\begin{tabular}{ccc}
\hline 4 & 0.131446 & $0.1348 / 0.1281$ \\
5 & 0.119373 & $0.1277 / 0.1110$ \\
6 & 0.133337 & $0.1382 / 0.1285$ \\
\hline \hline Combined Flights & 0.132458 & $0.1350 / 0.1300$ \\
\hline
\end{tabular}

Table 7.8: Dirty Drag Polar Repeatability Testing : $C_{1}$ Regression Coefficient

\begin{tabular}{ccc} 
Flight Number & $C_{1}$ Estimate & $95 \%$ Confidence Interval \\
\hline 4 & 0.004732 & $0.0087 / 0.0007$ \\
5 & 0.056782 & $0.0681 / 0.0454$ \\
6 & 0.014184 & $0.0211 / 0.0072$ \\
\hline \hline Combined Flights & 0.007363 & $0.0108 / 0.0039$ \\
\hline
\end{tabular}

Table 7.9: Dirty Drag Polar Repeatability Testing : $C_{2}$ Regression Coefficient

\begin{tabular}{ccc} 
Flight Number & $C_{2}$ Estimate & $95 \%$ Confidence Interval \\
\hline 4 & 0.113344 & $0.1151 / 0.1115$ \\
5 & 0.080817 & $0.0846 / 0.0770$ \\
6 & 0.118783 & $0.1212 / 0.1164$ \\
\hline \hline Combined Flights & 0.123563 & $0.1248 / 0.1223$ \\
\hline
\end{tabular}


Figure 7.13 shows combined drag polars for both dirty and clean configurations.

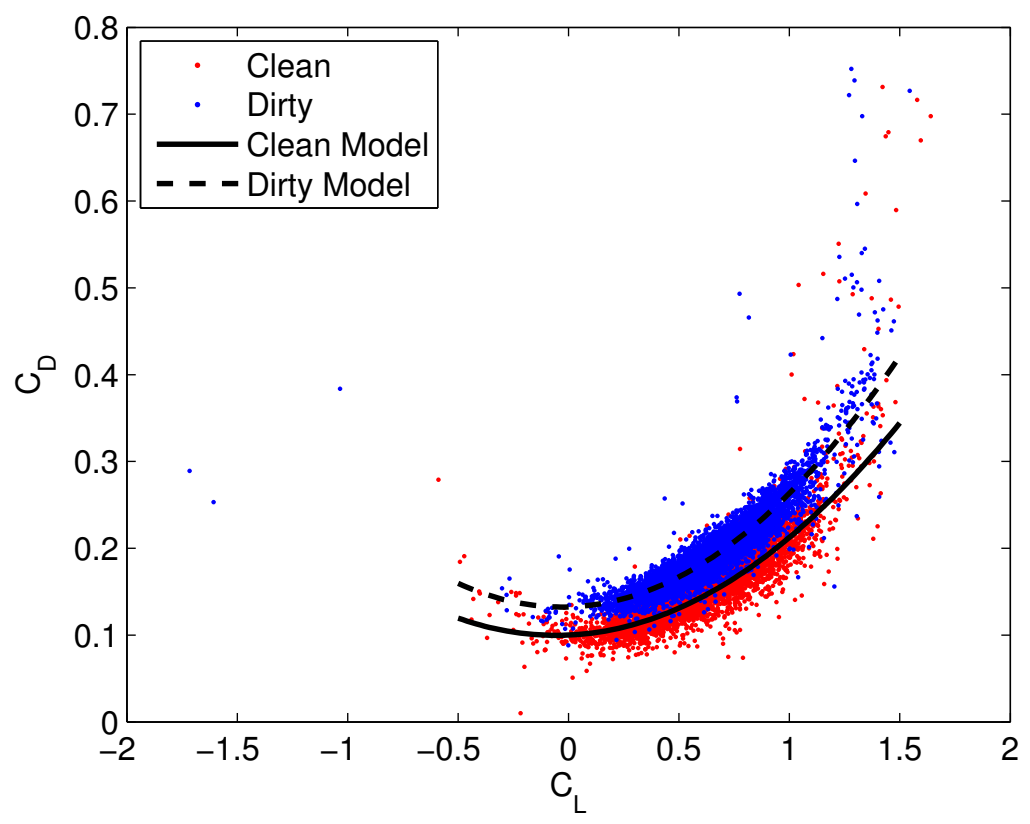

Figure 7.13: Clean vs. Dirty Drag Polar

The combined regression model for the dirty configuration is shown in Equation 7.4.1, and the combined regression model for the clean configuration is shown in Equation 7.4.2.

$$
\begin{aligned}
& C_{D_{D I R T Y}}=0.123563 C_{L}^{2}+0.007363 C_{L}+0.132458 \\
& C_{D_{C L E A N}}=0.101002 C_{L}^{2}+0.011454 C_{L}+0.100037
\end{aligned}
$$

The difference between the clean parasite drag coefficient $\left(C_{D_{0}}=0.100037\right)$ and the dirty parasite drag coefficient $\left(C_{D_{0}}=0.132458\right)$ is the contribution to the vehicle's parasite drag from the trailing cone. This amounts to $C_{D_{0, C O N E}}=0.0324$, which is $6 \%$ higher than the value previously estimated, and well within the error of the source data.

The lift curve for both dirty and clean flight tests is shown in Figure 7.14. The slopes are constant as is expected, since the lift curve slope is not a function of parasite drag. Note 
that the dirty data tends to occur at a slightly higher $C_{L}$ value, since the vehicle with more drag spends more time at a slower speed.

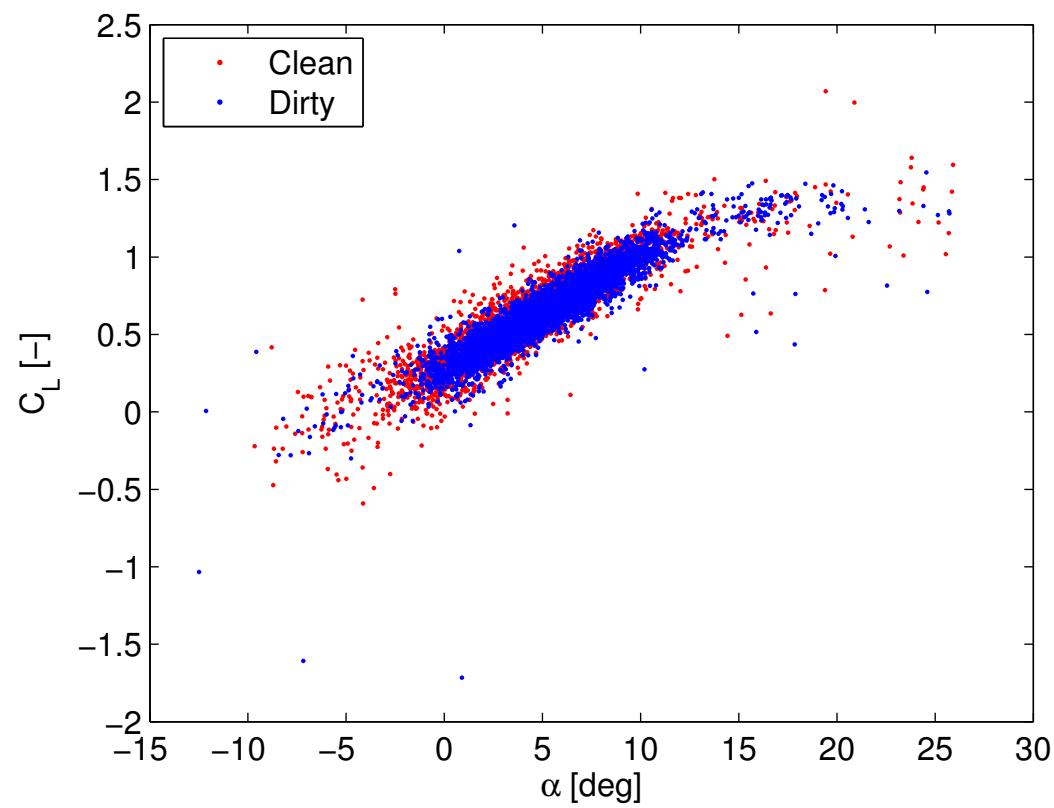

Figure 7.14: Clean vs. Dirty Lift Curve 


\subsection{Summary}

A flight data computer capable of measuring a small UAVs aerodynamic forces was developed. The system utilizes an Arduino Due as the main flight computer, and integrates both sensors necessary for aerodynamic force calculation, and additional sensors that provide interesting information about the vehicle. The system was manufactured in a PCB form to keep reliability high, integrated into a 0.60-size electric Piper Cub, and validated for accuracy and repeatability. The flight tests showed a $C_{D_{0}}$ accuracy of $6 \%$ of the trailing cone's estimated drag coefficient, and a zero lift angle of attack accuracy of less than $1 \%$ error compared to that estimated by XFOIL. The lift curve slope estimated from combining flight data with XFOIL analysis was, on average, accurate to 17\%. Future work could include a sensor fusion algorithm, which would be developed to combine inertial sensors with the air data system and other available sensors in a manner similar to other current research, ${ }^{[33],[34]}$ thus giving full situational awareness to the UAS. This situational awareness could allow stability and control derivative estimation, which the aircraft designer could use to size tail and control surfaces. In-flight dynamic thrust estimation is also possible, and could be validate against propeller data available from the University of Illinois at Urbana-Champagne. ${ }^{[35]}$ Of most interest to the other, future work could utilize this thesis to estimate the lift and drag impact of difference vehicle configurations to quantitatively make trade studies early in the conceptual design phase. This could include configuration level trades, or subsystem level (no landing gear, normal gear, gear with wheel pants, retractable gear,etc.) trade studies, which could dramatically increase the current small scale aerodynamic knowledge base at Cal Poly. 


\section{Bibliography}

[1] Mike1024. Ecec enu longitude latitude relationships. Wikipedia, February 2010.

[2] MLWatts. Hawker tempest mark ii three view. Wikipedia, September 2012.

[3] Olivier Clynen. 737 ng winglet effect simplified. Wikipedia, June 2012.

[4] Sighard F Hoerner. Fluid-dynamic drag: practical information on aerodynamic drag and hydrodynamic resistance. Hoerner Fluid Dynamics, 1965.

[5] Daniel P Raymer et al. Aircraft design: a conceptual approach, volume 3. American Institute of Aeronautics and Astronautics, 1999.

[6] Leland Malcolm Nicolai and Grant Carichner. Fundamentals of aircraft and airship design, volume 1. Amer Inst of Aeronautics \&, 2010.

[7] Jan Roskam. Airplane design. DARcorporation, 1985.

[8] Sighard F Hoerner and Henry V Borst. Fluid-dynamic lift: Practical information on aerodynamic and hydrodynamic lift. NASA STI/Recon Technical Report A, 76:32167, 1975.

[9] Vladislav Klein and Eugene A Morelli. Aircraft system identification: theory and practice. American Institute of Aeronautics and Astronautics Reston, VA, USA, 2006.

[10] Jan Roskam. Airplane flight dynamics and automatic flight controls, design. Analysis and Research Corporation, Lawrence, KS, 2001.

[11] Rudolph Emil Kalman. A new approach to linear filtering and prediction problems. Transactions of the ASME-Journal of Basic Engineering, 82(Series D):35-45, 1960. 
[12] Greg Welch and Gary Bishop. An introduction to the kalman filter, 1995.

[13] Leeland Nicolai. Estimating r/c model aerodynamics and performance. White paper, Society of Automotive Engineers, 2009.

[14] Mark Drela. Xfoil: An analysis and design system for low reynolds number airfoils. In Low Reynolds number aerodynamics, pages 1-12. Springer, 1989.

[15] Ludwig Prandtl. Applications of modern hydrodynamics to aeronautics. National Advisory Committee for Aeronautics, 1923.

[16] Brian L Stevens and Frank L Lewis. Aircraft control and simulation. 2003.

[17] Stephen R Osborne. Transitions between hover and level flight for a tailsitter uav. PhD thesis, Citeseer, 2007.

[18] Atmel. Sam3x-sam3a series summary. Data Sheet 11057BSATARM13-Jul-12, Atmel, 2012.

[19] Atmel. 8-bit atmel microcontroller with 64k/128k/256k bytes in-systemprogrammable flash. Data Sheet 2549PAVR10/2012, Atmel, 2012.

[20] Analog Devices. Micropower, 3-axis,2 g/4 g/8 g digital output mems accelerometer. Technical report, 2012.

[21] U-Line. Easy-count counting scale data sheet. Technical report, U-Line.

[22] Honeywell Magnetic Sensors. Smart digital magnetometer hmr2300. Technical report, Honeywell, 2006.

[23] Honeywell. 3-axis digital compass ic hmc58831. Technical report, Honeywell.

[24] Talat Ozyagcilar. Calibrating an eCompass in the Presence of Hard and Soft-Iron Interference. Freescale Semiconductors, rev 3.0 edition, 042013. 
[25] Yury Petrov. Ellipsoid fit. Matlab File Exchange, 082013.

[26] InvenSense. Itg-3200 product specification revision 1.4. Technical report, 2010.

[27] All Sensors. Ds-0012 rev a. Technical report, All Sensors.

[28] All Sensors. Ds-0010. Technical report, All Sensors.

[29] Maxim Integrated. Ds18b20 programmable resolution 1-wire digital thermometer. Technical report, Maxim Integrated, 2008.

[30] Crystal Engineering, 708 Fiero Lane, Suite 9, San Luis Obispo, California 93401. nVision Operation Manual for Reference Recorder, 2013.

[31] Paroscientific. Model 745 high accuracy portable pressure standard data sheet. Technical report, Paroscientific.

[32] Doug Weibel. Proof of concept test - extremely accurate 3d velocity measurement with a ublox 6t module., December 2012.

[33] Matthew B. Rhudy; Trenton Larrabee; Haiyang Chao; Yu Gu; Marcello Napolitano. Uav attitude, heading, and wind estimation using gps/ins and an air data system. 2013.

[34] Seung-Min Oh and Eric N Johnson. Development of uav navigation system based on unscented kalman filter. In AIAA Guidance, Navigation and Control Conference, 2006.

[35] John B Brandt and Michael S Selig. Propeller performance data at low reynolds numbers. In AIAA Aerospace Sciences Meeting, AIAA 2011, volume 1255, 2011. 


\section{A.0 Data Acquisition System Usage}

This section documents the steps required for correct usage of the data acquisition system.

\section{A.1 Integration}

The main data acquisition system should be integrated into the flight test vehicle near the vehicle's center of gravity. The system should have one of its principal axes lined up roughly parallel to the vehicle's longitudinal axis. Servos should be connected to both the main board and the aircraft's receiver using a y-splitter with 2 male and one female connectors. If applicable, the servos should be connected according to the Table A.1.

\begin{tabular}{cc} 
Table A.1: Air Data System Setup \\
\hline Servo & PWM Pinout \\
\hline Throttle & D37 \\
Elevator & D38 \\
Rudder & D39 \\
Aileron (1/2) & D40 \\
Aileron (2/2) & D41 \\
Gear & D42 \\
Auxiliary $(1 / 2)$ & D43 \\
Auxiliary $(2 / 2)$ & D44 \\
\hline
\end{tabular}

If the additional digital pin-outs are being use for measuring servo signals, the " $+\mathrm{V}$ " jumper should not have a jumper on it. If using the digital pin-outs to run digital sensors or command servos, place the jumper on the appropriate voltage level setting.

The data acquisition system is powered through the barrel jack on the Arduino Due. The recommended operating voltage is between $7 \mathrm{~V}$ and $12 \mathrm{~V}$, with an absolute maximum of $16 \mathrm{~V}$. 
The power can be supplied using either a battery dedicated to the data acquisition system, or through a BEC from the ESC. If using the ESC's BEC, set the BEC output somewhere between $7 \mathrm{~V}$ and $12 \mathrm{~V}$. CRITICAL: ensure the red wire from the ESC does not pass to the receiver, as it is over-voltage for standard $\mathrm{R} / \mathrm{C}$ equipment.

The pressure board can be placed anywhere in the vehicle, as long as it can be connected to the main board. The pressure sensors should be attached to the five-hole probe as follows:

Table A.2: Air Data System Setup

\begin{tabular}{cccc}
\hline \hline Pressure Sensor & Port A & Port B & Measurement \\
\hline 0 & Tube 5 & Tube 4 & $\beta$ \\
1 & Tube 6 & Tube 1 & $q_{\infty}$ \\
2 & Tube 3 & Tube 2 & $\alpha$ \\
3 & n/a & Tube 6 & $P_{S}$ \\
\hline
\end{tabular}

Port A and Port B refer to the ports as labeled in Figure A.1, and the tube number refers to the five-hole probe tubes. These tube numbers increase as the tube length decreases: Tube 1 refers to the longest tube, Tube 6 refers to the shortest tube. 


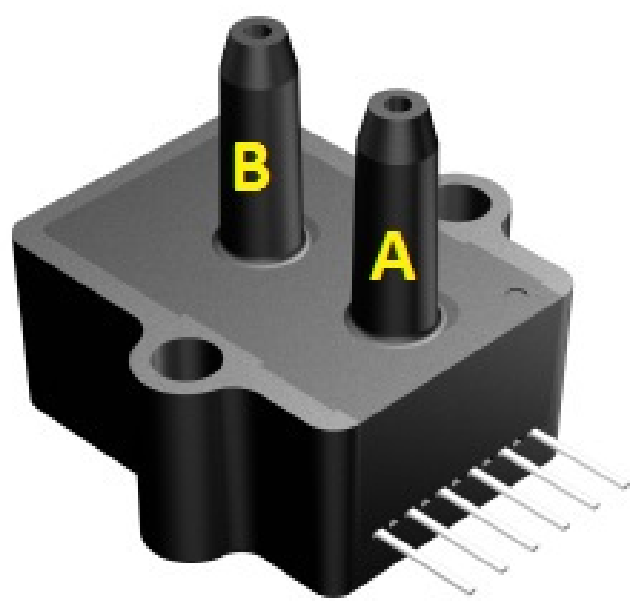

\section{Figure A.1: Port Description for Pressure Sensors}

The pressure tubing and temperature sensor line should be routed together to the air data boom and secured for flight. The temperature sensor's wiring is a standard servo extension. The air data boom's accelerometer wiring is a standard RJ-25 patch cable and should be routed in a separate bundle, as it will be removed before flight.

The air data boom itself should be integrated as far from aerodynamic effects as possible. For this thesis, the boom was mounted roughly one chord length in front of the leading edge of the wing and approximately halfway down the wing span. Other mounting locations could include out the aircraft's nose for a pusher vehicle, or on the vertical tail. The aluminum block accepts a 0.25 " carbon fiber tube as it's mounting interface, and this tube should be mounted roughly in-line with the airflow angle expected during flight. The temperature sensor can be taped to the tube. 


\section{A.2 Pre-flight Procedure}

The pre-flight procedure in this section must be completed in addition to all preparation listed in Section D.

1. With the transmitter turned on, power on the receiver.

2. Plug in the micro-USB cable into the main data acquisition board.

3. Ensure the micro-SD card is empty and formatted as FAT32, then insert it into the main data acquisition board.

4. Plug in the main data acquisition system's battery pack or the BEC. CRITICAL: ensure the red wire does not connect the BEC to the receiver.

5. Plug the pressure sensor board into the main data acquisition board.

6. Plug in the air data system's RJ-25.

7. Upload the calibration routine to the main board, and follow the prompts to calibrate the gyroscope, accelerometer, and magnetometers.

8. Place a wind blocker (Daisy/Solo cups work) over the five-hole probe and calibrate the pressure sensors, using the calibration routine.

9. Load the main data acquisition script onto the main data acquisition board.

10. Turn on serial output and check that all sensors are functioning properly.

11. Initialize the micro-SD card.

12. Turn off serial output.

13. Turn on data logging and verify the system is saving data.

14. Remove the micro-USB cable and the air data system's RJ-25 cable. 
15. Plug in the main propulsion battery pack.

The data acquisition system is now ready for flight.

\section{A.3 Flight test plan}

When using the data acquisition system for drag polar estimation, a better model estimate is possible with proper flight maneuvers. The main technique to improve the model is to vary speeds as much as possible, which allows more of the drag polar to be flown. Multiple glides that begin at the highest speed possible should be completed. To achieve the highest initial gliding speed, the aircraft should be put into a dive at full throttle, with plenty of available altitude. Throttle should then be cut, and the dive should briefly continue. After a few seconds of diving, the aircraft should be leveled off and altitude should be maintained, until the vehicle stalls. Repeating this process will provide a large sweep of the flight envelope.

Inverted flight can also help fill out the section of the drag polar that occurs below $C_{L_{\text {min }}}$. However, inverted flight should occur at high speeds, to avoid the nonlinear section of the inverted airfoil.

\section{A.4 Post-Flight}

Immediately after the vehicle lands, the vehicle's propulsion pack should be unplugged. Next, unplug the data acquisition system's battery pack, remove the micro-SD card, and save the data to a computer. Optionally, after the flight is complete, a second round of zero offset calibration data may be acquired. This set ensures that any drift that occurred during the flight is accounted for.

Once the data is saved to a computer, the data analysis GUI can be used to quickly process the data. The user interface can estimate a drag polar using the following steps:

\section{Run fileReader.m.}


2. Click the "Load Data" button to load flight data. If you'd like an ASCII data file, choose yes on the prompt.

3. Select the pressure calibration file.

4. Select the accelerometer/gyroscope calibration file.

5. Select the magnetometer calibration file.

6. Select the air data system alignment calibration file.

7. Input the reference area in the $S_{R E F}$ box and the weight in the Weight box.

8. Click "Ignore Data With Thrust."

9. Click "Display" in the Quadratic Fit box.

Google Earth plots are also available by clicking the "Google Earth" button. The "X Axis" and "Y Axis" drop down menus are also available, which allows any signal to be plotted against any other signal.

\section{A.5 Embedded Software Protocol}

The main data acquisition code allows the user to interface with the Arduino through a serial text interface. A list of available commands for the main data acquisition script, along with their intended functions, is shown in Table A.3. 
Table A.3: Available Commands for mainScript.ino

\begin{tabular}{|c|c|}
\hline Command & Utility \\
\hline \hline dataOn & Turn on data logging to SD card \\
\hline dataOff & Turn off data logging to SD card \\
\hline initSD & Initialize SD card \\
\hline serialOn & Turn on serial output \\
\hline serialOff & Turn off serial output \\
\hline$?$ & Help menu \\
\hline
\end{tabular}

Note that all commands must be sent with a start character ('\#') and an end character ('\&'.) A proper command to turn on serial output would then be '\#serialon\&'.

The calibration code also interfaces with the Arduino using a text interface. The calibration script's commands are shown in Table A.4.

Table A.4: Available Commands for calibration.ino

\begin{tabular}{|c|c|}
\hline Command & Utility \\
\hline \hline accelGyro & Calibrate accel for slope and offset, gyro for offset. Saved to ACCLGYRO.clb. \\
\hline pressure & Calibrate offset of pressure sensors. Saved to PRESSURE.clb. \\
\hline mag & Calibrate magnetometers for hard- and soft-iron effects. Saved to MGNTMTRS.clb. \\
\hline$?$ & Help menu \\
\hline
\end{tabular}

Note that, like the main script, all commands must be sent with a start character ('\#') and an end character ('\&'.) A proper command to calibrate the air data system alignment would then be '\#pressure\&'. 


\section{B.0 Lessons Learned}

There were numerous lessons learned during this thesis. Many of them are presented here, in an effort to reduce future learning curve pains.

\section{Understand exactly what each sensor does.}

During this thesis, there were issues with the exact measurement a sensor makes. For instance, accelerometers do not measure accelerations. They measure the accelerations resisting freefall, or in other words, the difference between gravity and accelerations. As another example, magnetic compasses are extremely different than magnetometers, and this difference should be known before purchasing either. It is absolutely critical to understand what each sensor measures before sensors are purchased.

\section{Continue to read papers while working.}

A literature review at the beginning of a research project is important to help frame the problem definition and scope. However, whenever there a problem occurs, it is incredibly helpful to continue reading papers and see how the problem has been solved in the past.

3. Simulation is in only as good as the inputs and assumptions. Know what they are, and how they affect the results.

This lesson was directly coupled to the vector and orientation issue. Originally, the assumption made was that Euler angles were required for drag polar estimation. Then, a gliding flight assumption was introduced, which meant Euler angles were unnecessary to begin with. However, simulation work was continued to estimate Euler angles. Another assumption was made that, because most data sheets for magnetometers state roll and pitch accuracy specifications, there must be a way to get Euler angles from them. So

work was stopped, and it was assumed Euler angles could be calculated. This meant 
the assumptions going into the simulator were bad, and produced erroneous results, but because assumptions were not clearly stated, the mistake was not caught until late in the research.

\section{Digital sensors are not necessarily better.}

It is tempting to use all digital sensors since many microcontrollers have a lot more digital I/O pins than analog I/O pins. The time it takes to understand the digital interface is substantial when compared to analog voltages. Other digital effects, such as line noise, were experienced in this research. This lesson is not that analog sensors are better, but rather that an understanding of the pros and cons of each is required.

\section{Understand the communication protocol and the system impact it might have.}

A significant portion of the system sampling time is consumed by transmitting and reading unique commands to the pressure sensors. The unique commands required 40 bytes per read/write, while the non-unique commands required only 15 bytes. The 40 bytes of read/write time per sensor multiplied by the four sensors meant the pressure sensors required as much read/write time as all other sensors combined, and grew at a rate of 2.7 times the number of sensors sharing the line. That is in direct comparison to the HMR-2300 magnetometer, which had a single unique and non-unique read command of 5 bytes, and each response (unique or not)was 7 bytes.

\section{Data sheets are your friend.}

Data sheets seem daunting at first, especially with limited exposure to them. It is much better to fully understand the data sheet, rather than to skim over it and waste time figuring out why the system is not working as expected.

\section{Hardware integration is difficult and necessary.}

There were two in-flight issues that came as a result of this research hardware. The first occurred when a jumper wire short circuited the control system of the vehicle. This 
resulted in vehicle loss. If the PCB had been complete and the jumper cable was not necessary, this crash most likely would not have occurred. The second issue was when the RPM signal line came unplugged from the system. This did not result in a crash, but made the data from that flight unusable. Both problems came from interfacing the fully-integrated PCB with the outside world. Previous experience has shown much lower flight success with systems not fully integrated into a PCB design. The lesson learned here is that, while difficult and timely, an integrated PCB will lead to a much more reliable and robust system.

\section{Avoid surface mounted components whenever possible.}

A lot of effort and money was put into surface mounting the sensors which could be surface mounted. Eventually, this was successful. However, it required hand soldering to be done by a professional, and half of those boards had thermal damage to the sensors which made them unusable. There was no requirement driving the system to be so small that surface mount devices were necessary. Unless necessary, always use breakout boards.

\section{Always buy the developer kit first.}

When protoyping something, the developer kit is your friend. It will save substantial headache and time, and is well worth the price difference. 


\section{C.0 Wiring Schematics}

The following documents the wiring of the circuit boards. The actual Eagle files are available in /eagle/.

Figure C.1: Arduino Due Flight Data Recorder v3.20BOB Schematic
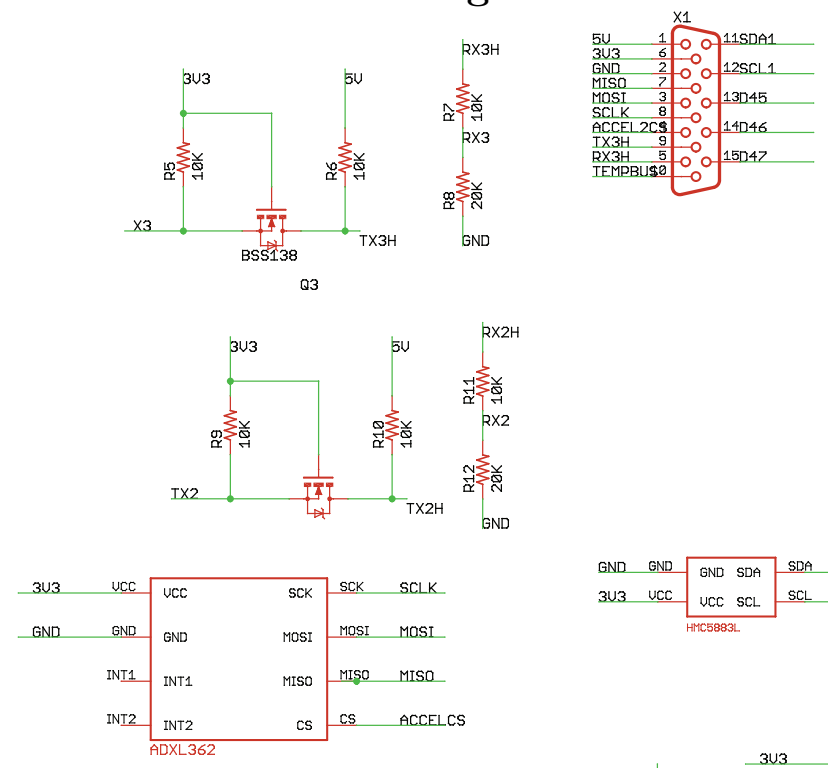

$$
\begin{array}{ll|l|l|ll}
\text { GND } & \text { GND } & \text { END } & \text { SDA } & \text { SDA } & \text { SDA1 } \\
\hline 3 U 3 & \text { UCC } & \text { UCC } & \text { SCL } & \text { SCL } & \text { SCL } 1 \\
\hline \text { UHC5883L } & &
\end{array}
$$
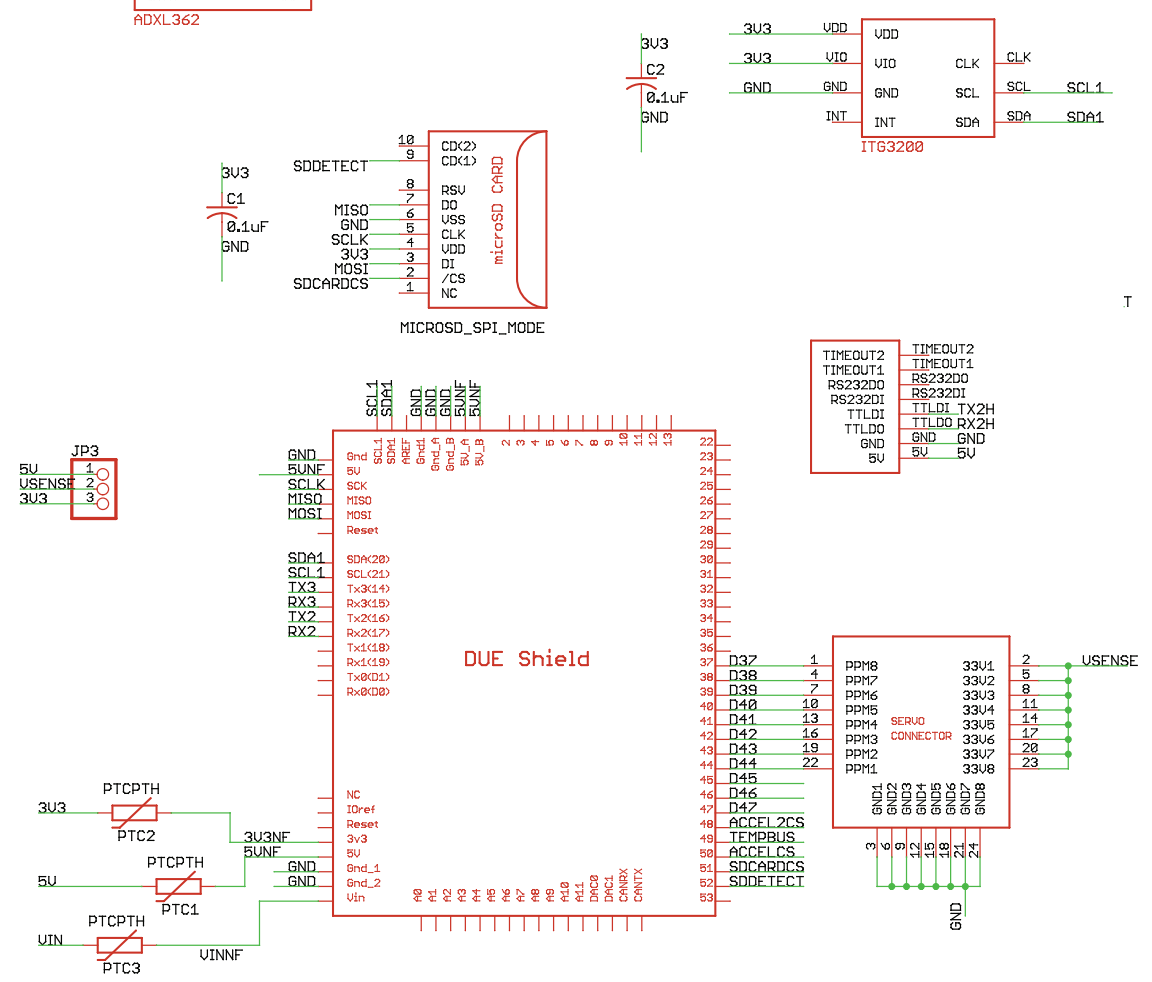
Figure C.2: Arduino Due Flight Data Recorder v3.20BOB Layout

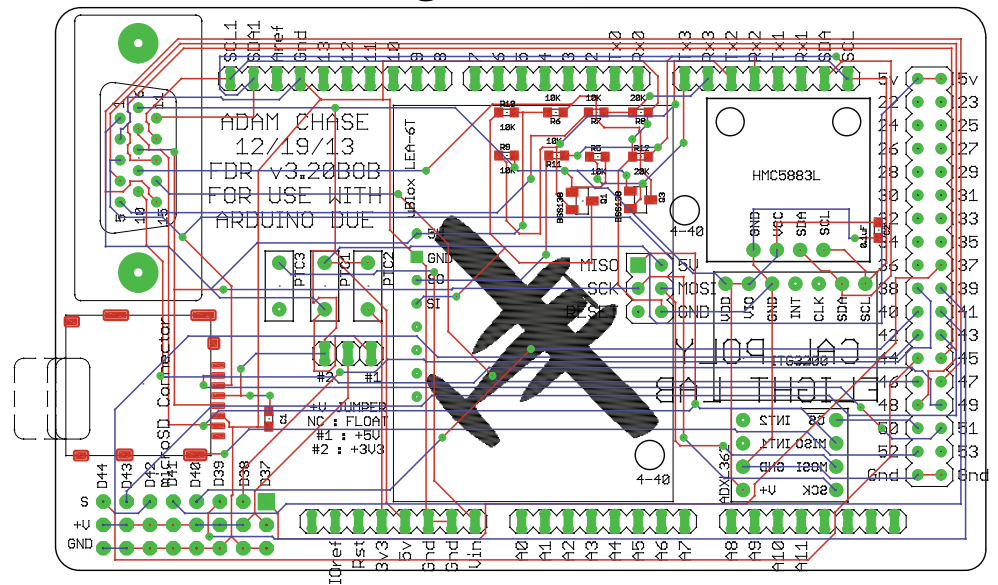

Figure C.3: Pressure Board v2.20 Schematic

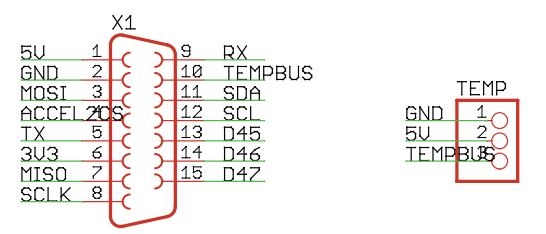

$54 \underbrace{\mathrm{R} 1}_{2.2 \mathrm{~K}} \mathrm{TX}$
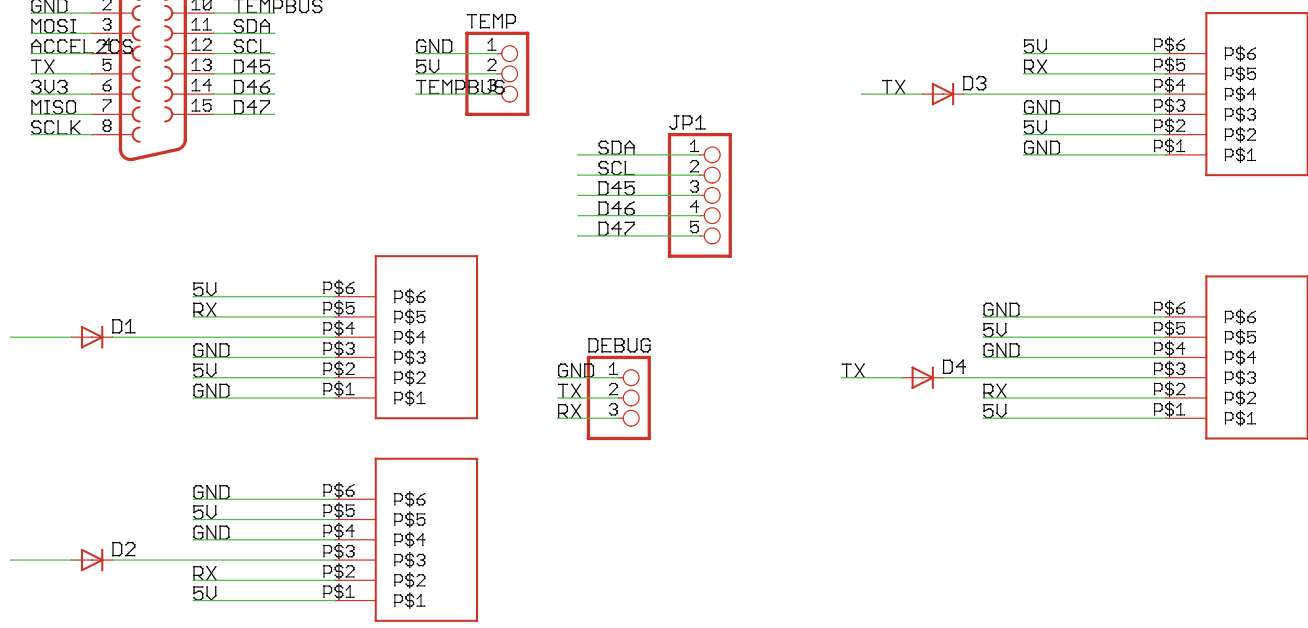

\begin{tabular}{ll|l|}
$3 U 3$ & $P \$ 6$ & $P \$ 6$ \\
GND & $P \$ 5$ & $P \$ 5$ \\
MISO & $P \$ 4$ & $P \$ 4$ \\
MOSI & $P \$ 3$ & $P \$ 3$ \\
SCLK & $P \$ 2$ & $P \$ 2$ \\
ACCEL2C $\$ 1$ & $P \$ 1$ \\
\hline
\end{tabular}


Figure C.4: Pressure Board v2.20 Layout

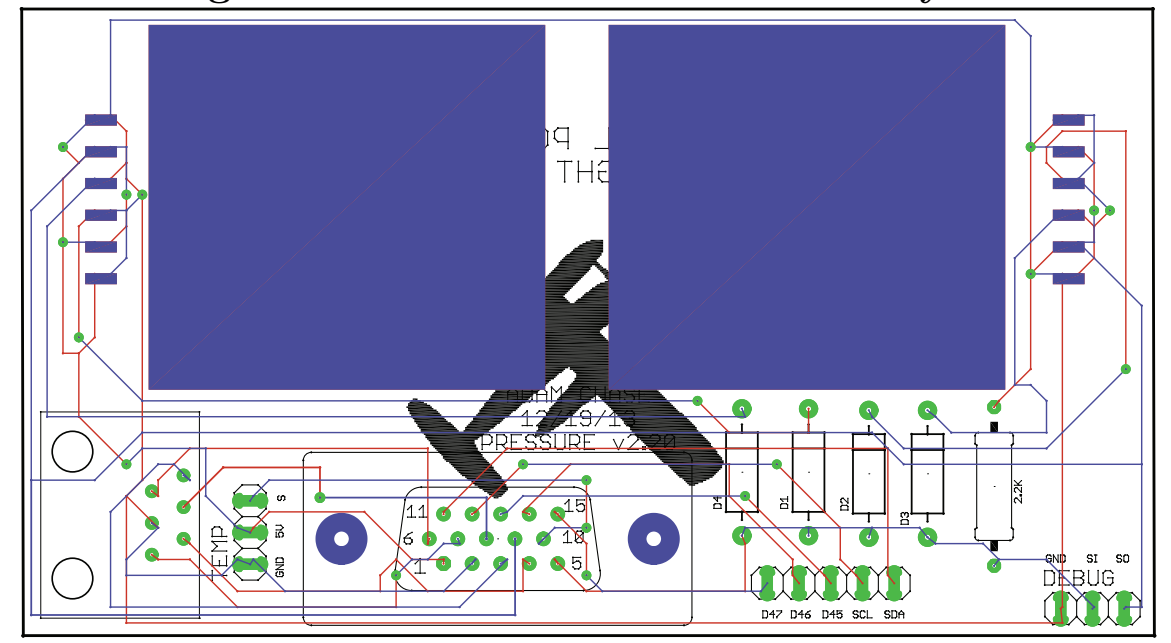




\section{D.0 Flight Test Procedure}

This section documents proper flight test procedure, which has been learned from extensive flight testing experience and many crashed vehicles. It is split into three main time periods:

1. Pre-Flight Preparation

2. Flying Field Procedure

\section{Post-Flight}

The testing procedure is split into these time categories to ensure the testing is efficient and that any unforeseen circumstances can be dealt with quickly. Specifically, this guide applies to the Cal Poly Flight Lab testing a vehicle at the Cal Poly Educational Flight Range.

\section{D.1 Pre-Flight Preparation}

The preparation work required for a test flight is often overlooked, and this section will document how to effectively prepare to flight test a vehicle. The main purpose of pre-flight preparation is to minimize the possibility of problems that might force a test to be canceled after already going to the field.

\section{D.1.1 Day Before Test}

The days before a flight test are critical to ensuring the test is completed successfully. The following should be done at least one day before the the test is scheduled:

1. Charge all flight battery packs, including receiver or auxiliary packs. NOTE: receiver packs should be used whenever possible. Avoid using the BEC on the ESC, and cut the red wire on the servo extension between the ESC and the receiver. 
2. Charge the transmitter battery.

3. Ensure the airframe is structurally sound (wing tip test minimum.)

4. Verify radio system communicates properly, and the correct fail-safe is in place. Important: If the receiver has been used by Design/Build/Fly, the fail-safe must be changed to normal mode.

5. Verify control surface deflections matches desired directions, and all radio mixes work.

6. Verify the motor/propeller spin in the correct direction.

7. Pack a flight box, containing any necessary tools (recommend: screw drivers; masking, painter's, and strapping tape; razor blades; CA glue and kicker; spare propellers; paper and pencil; sunglasses; as a minimum.)

8. Check the weather. The closest monitoring station to the field is KCASANLU17.It is generally better to flight test early in the morning, since there will be less people at the field, winds will be calmer, and the sun won't be as harsh in the pilot's eyes.

9. Check the SLO Flyers' flight schedule. Some days are reserved for certain events (glider competitions, etc.) and these need to be worked around.

10. Make sure all required personnel know when and where to meet, and there is sufficient transportation to get to the field.

11. Create flight documentation that clearly lays out the test goals and how they will be accomplished. Print copies for all personnel.

\section{D.1.2 Day Of Test}

The morning of the flight test, the person responsible for the test should arrive early enough to accomplish the following, before leaving campus: 
1. Pack flight batteries into flight box, including receiver and auxiliary packs.

2. Pack battery charging equipment, with adapters and leads, if necessary.

3. Pack transmitter into box.

4. Double check control surface deflections and radio link.

5. Do a full system check, potentially including a short taxi test in the quad.

6. Do a final check that all equipment made it into vehicles, before leaving the lab.

\section{D.2 Flying Field Procedure}

With the pre-flight preparation completed, the testing at the flying field should be fairly event free. Any problems that occur should be either fixable with the minimum supplies in the flight box, or the test should be canceled to minimize risk, and repairs done at the lab. Specific procedures at the flight field will depend on the test being conducted, but below are steps that apply to nearly all tests before flight.

1. Verify structural integrity using a wing tip test.

2. Verify motor/propeller are spinning in the correct direction.

3. Verify control surface deflections match desired directions.

4. Verify radio link and fail safe mode by completing a range check.

5. Verify center of gravity is at an appropriate location.

6. Create flight timer on the transmitter so the pilot knows how long the aircraft has been flying.

7. Document any required data before the flight, such as aircraft weight and geometry. 
8. Document current weather conditions (wind speed and direction, temperature, humidity, barometric pressure) using the Kestrel portable weather station.

\section{D.3 Post-Flight}

Upon successful completion of a flight test, all data should be saved to a computer. The flight test cards, pre-flight data documentation, and any notes should be scanned and saved with the flight data. This data should be archived in a .zip file, with the date attached.

If the test flight resulted in a crash, any available data should still be saved. Any video or pictures of the flight should be saved as well, to aid in isolating what caused the crash. If useful, pictures should be taken of the crash site. Afterwards, all pieces of the aircraft should be returned to the lab, where the root cause of the accident should be determined.

After the root cause has been determined, remove all electronics from the vehicle, including batteries, speed controller, motor, servos, and receiver. If the vehicle was a complete loss or went into water, through these components away: do not return to lab supplies. If the vehicle was not a complete loss, verify all components work properly. 


\section{E.0 Sample System Ouput}

This appendix contains graphs representing typical outputs from the data acquisition system, shown in strip chart format.

\section{E.1 Sample System Ouput - Raw Data}

Figure E.1: accelX vs. Time

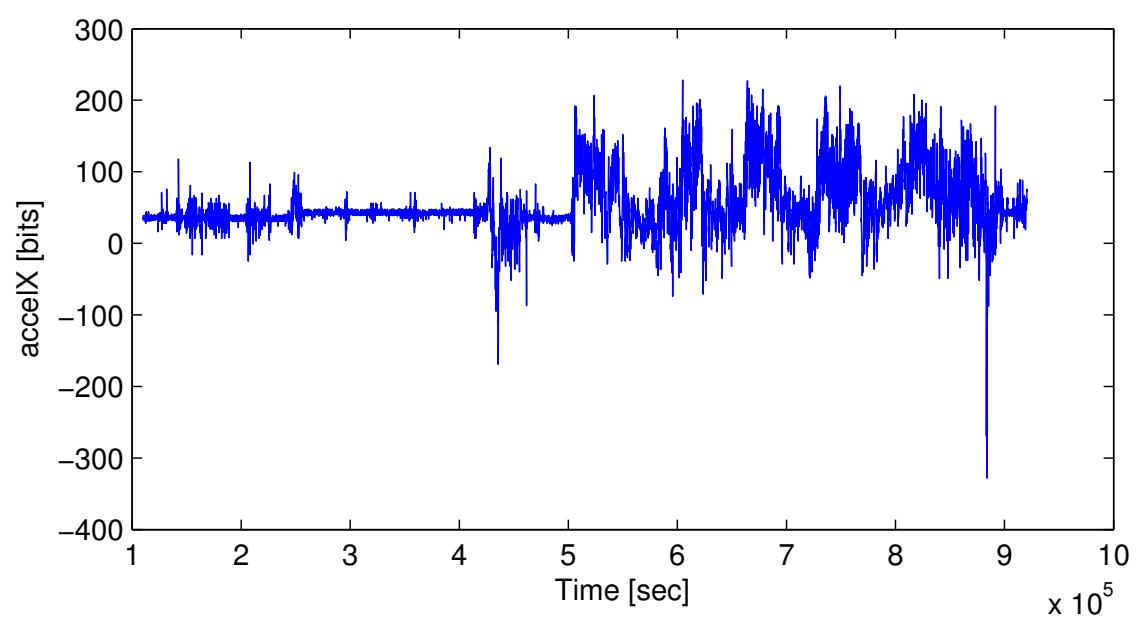

Figure E.2: accelY vs. Time

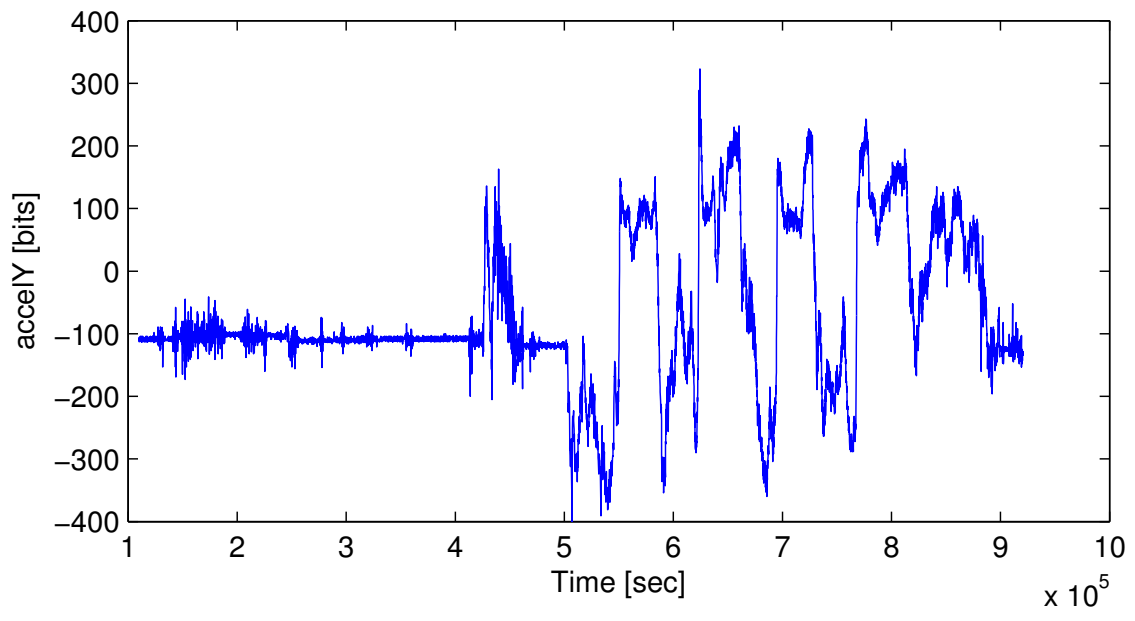


Figure E.3: accelZ vs. Time

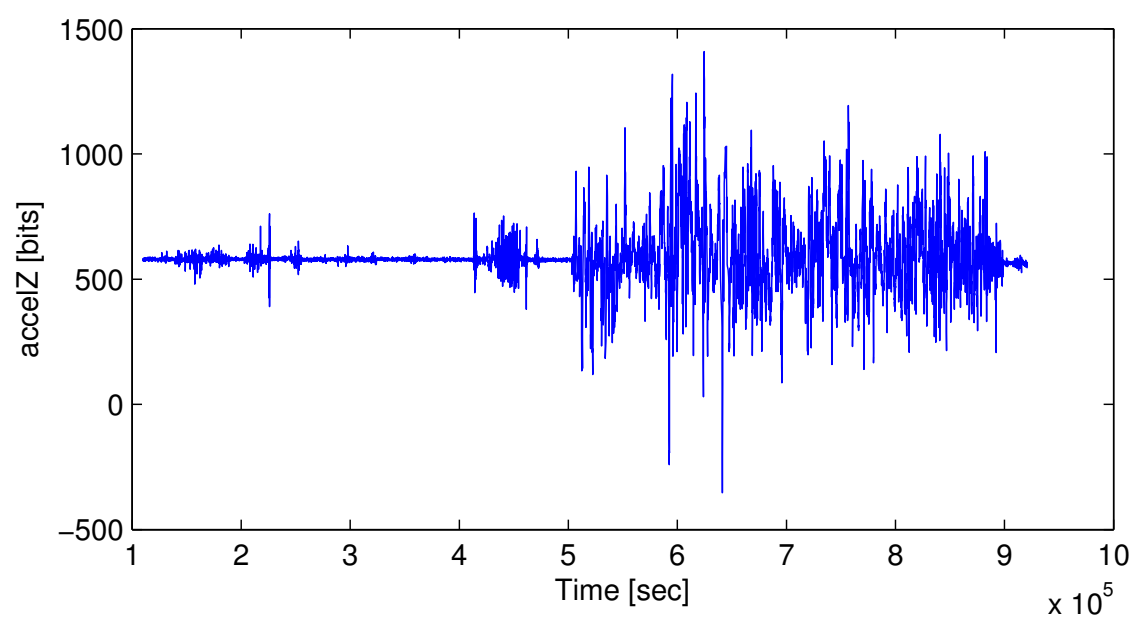

Figure E.4: gyroX vs. Time

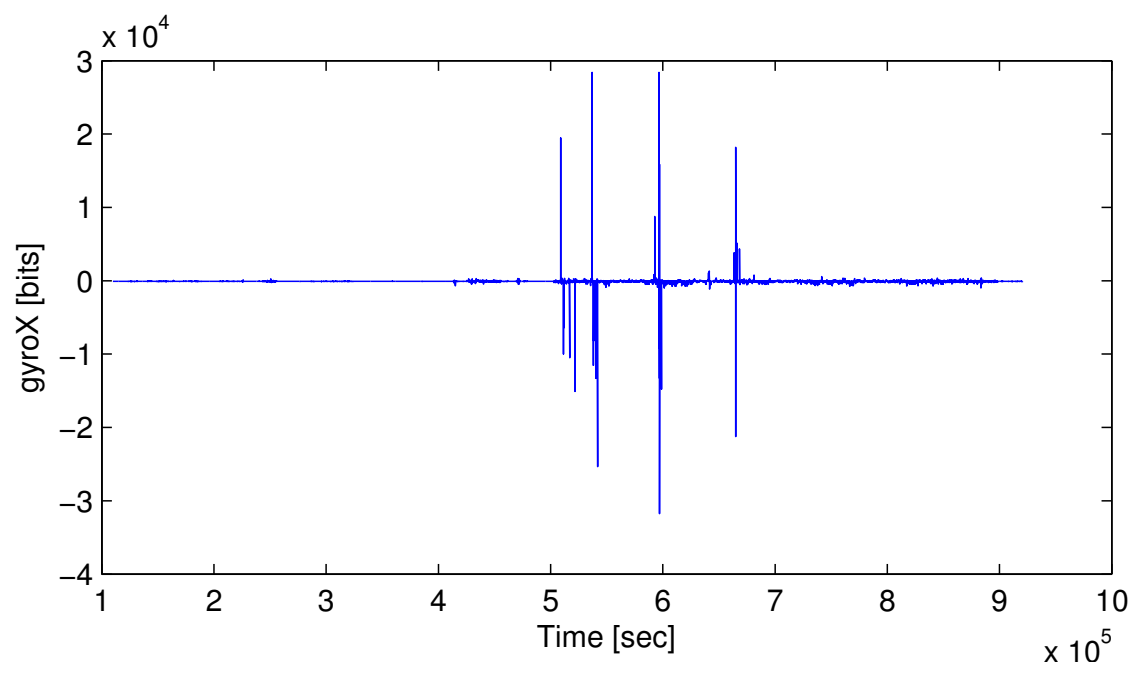


Figure E.5: gyroY vs. Time

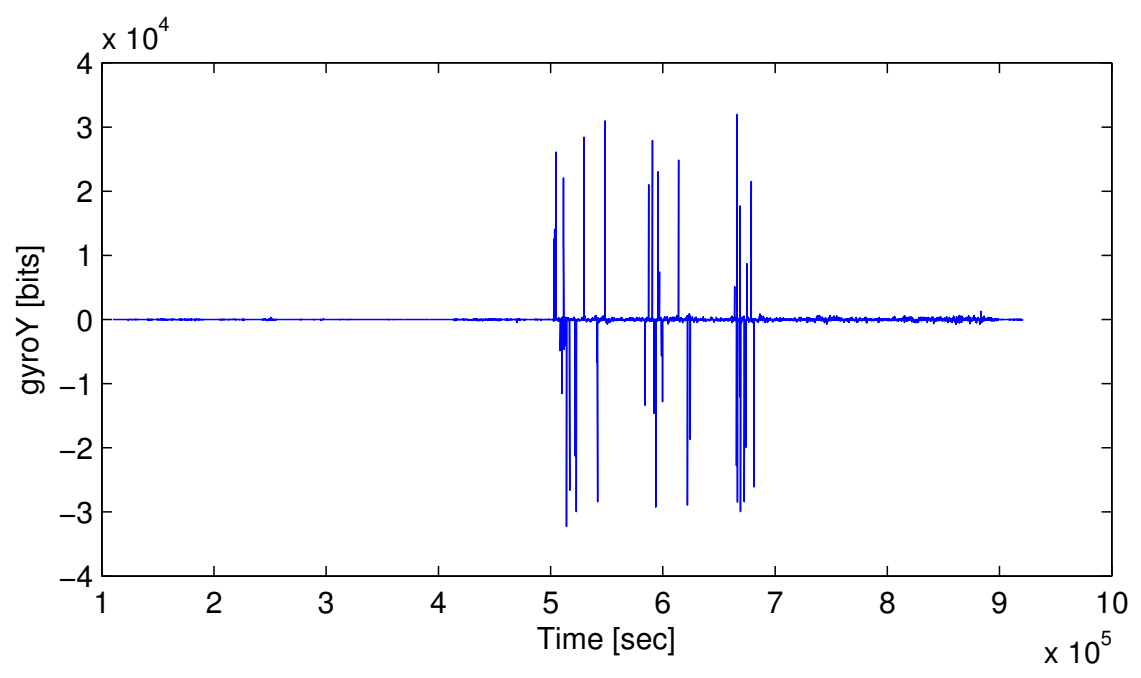

Figure E.6: gyroZ vs. Time

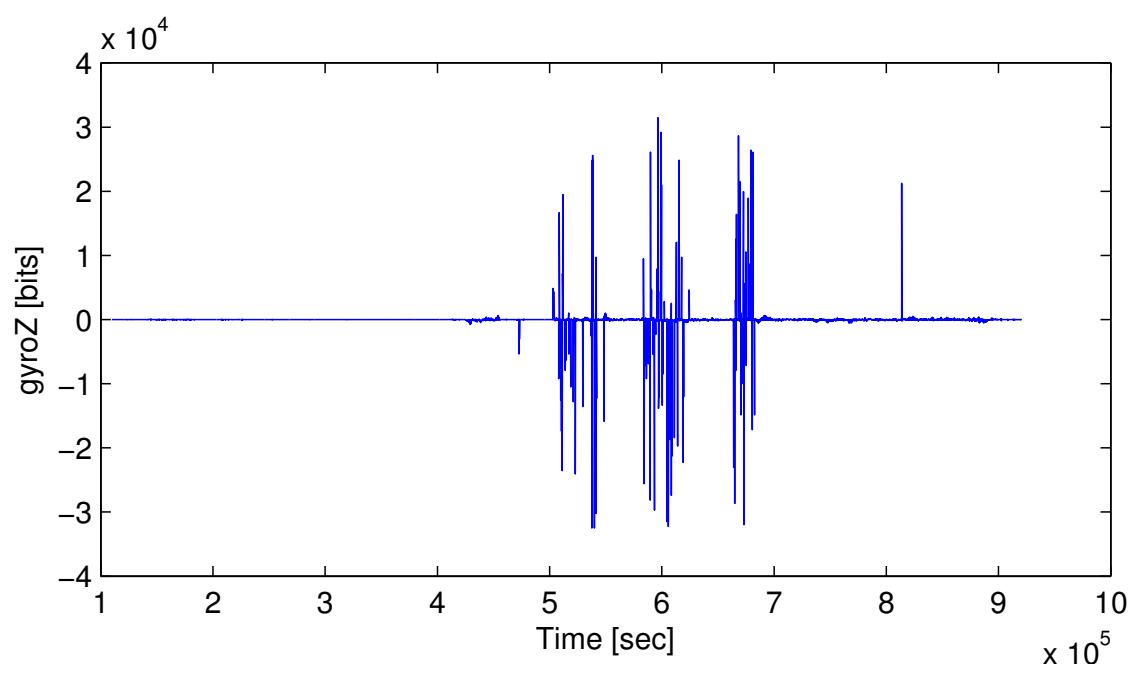


Figure E.7: $\operatorname{magX}$ vs. Time

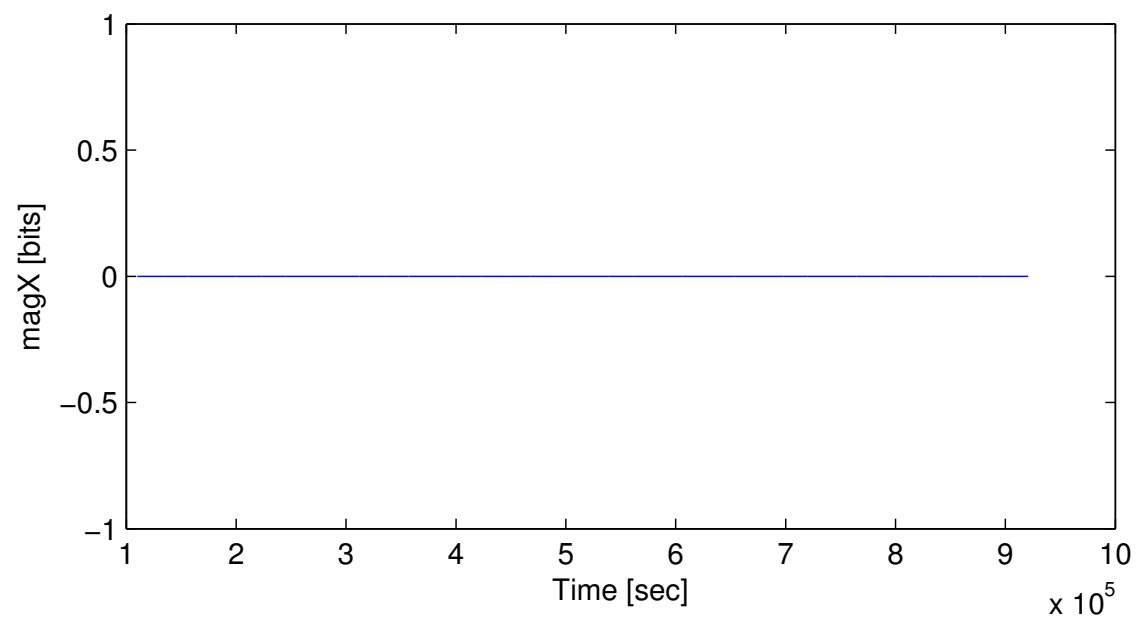


Figure E.8: magY vs. Time

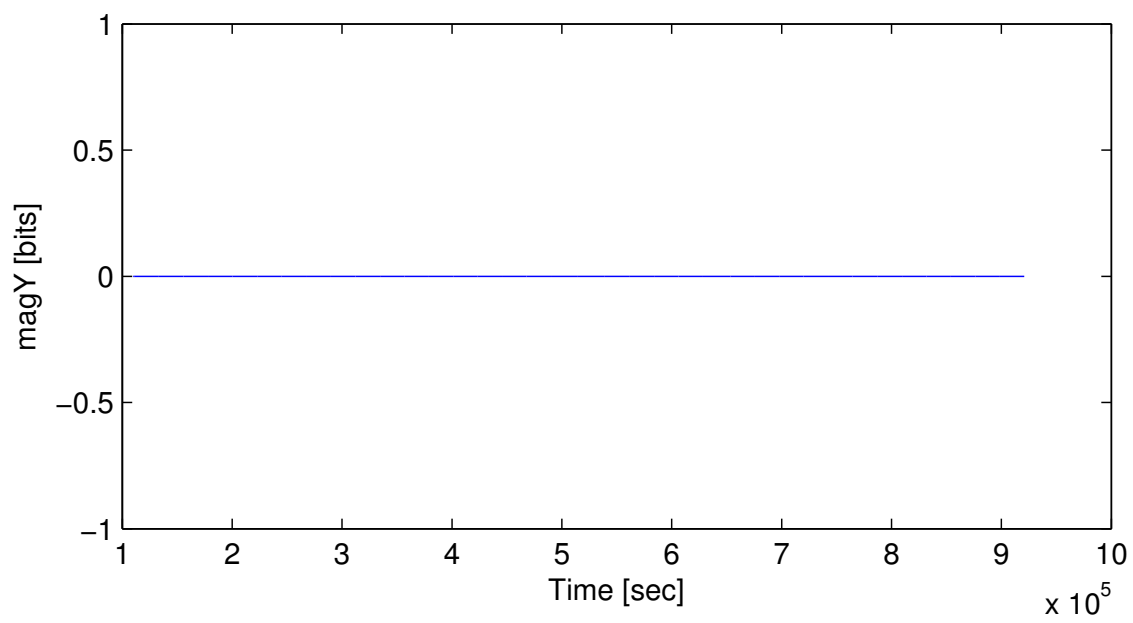

Figure E.9: $\operatorname{magZ}$ vs. Time

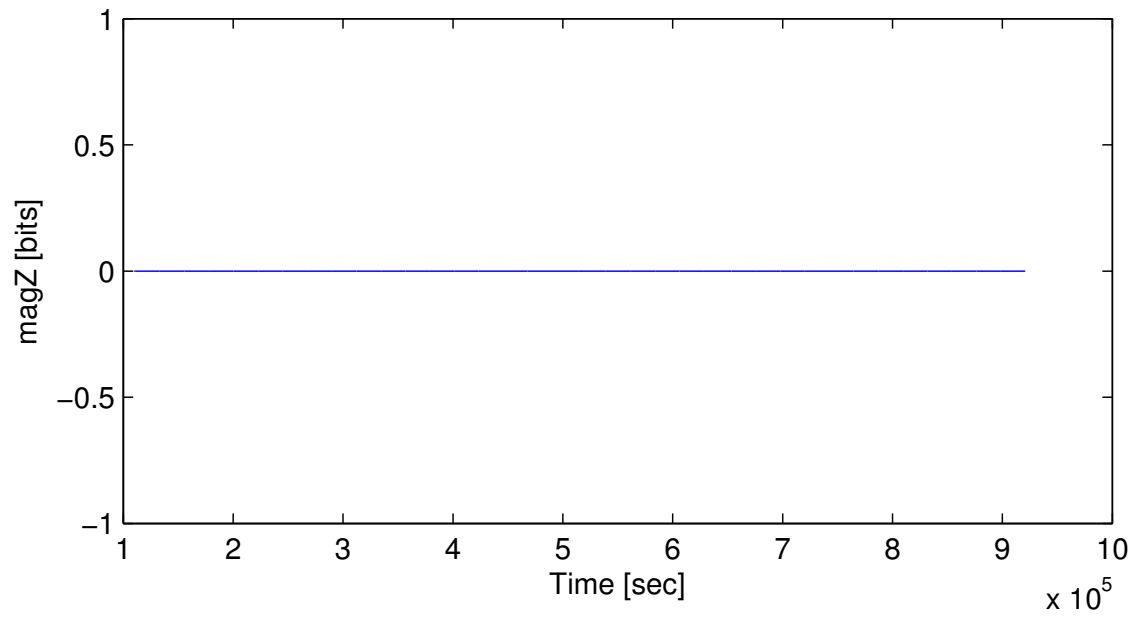


Figure E.10: hmcX vs. Time

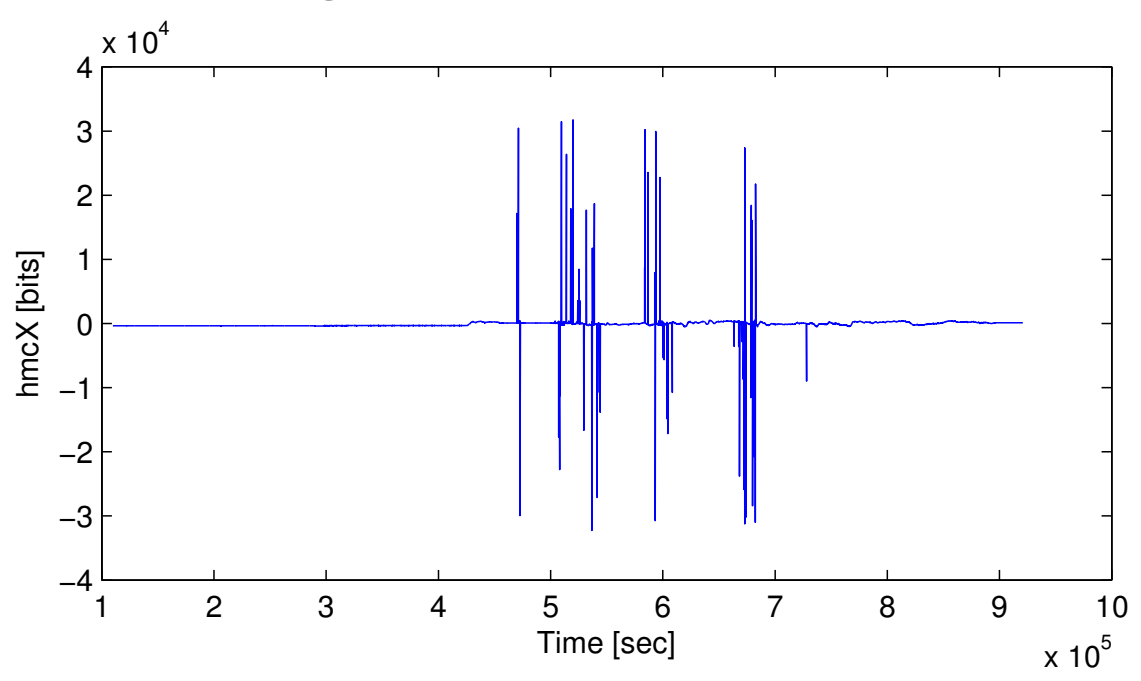

Figure E.11: hmcZ vs. Time

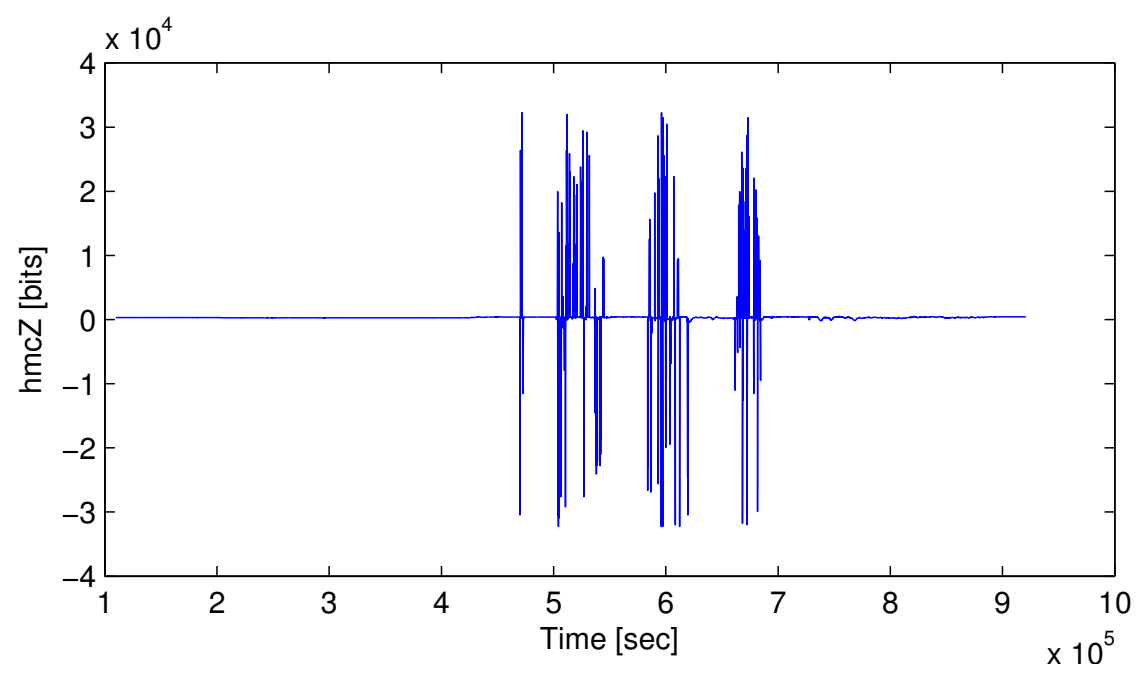


Figure E.12: hmcY vs. Time

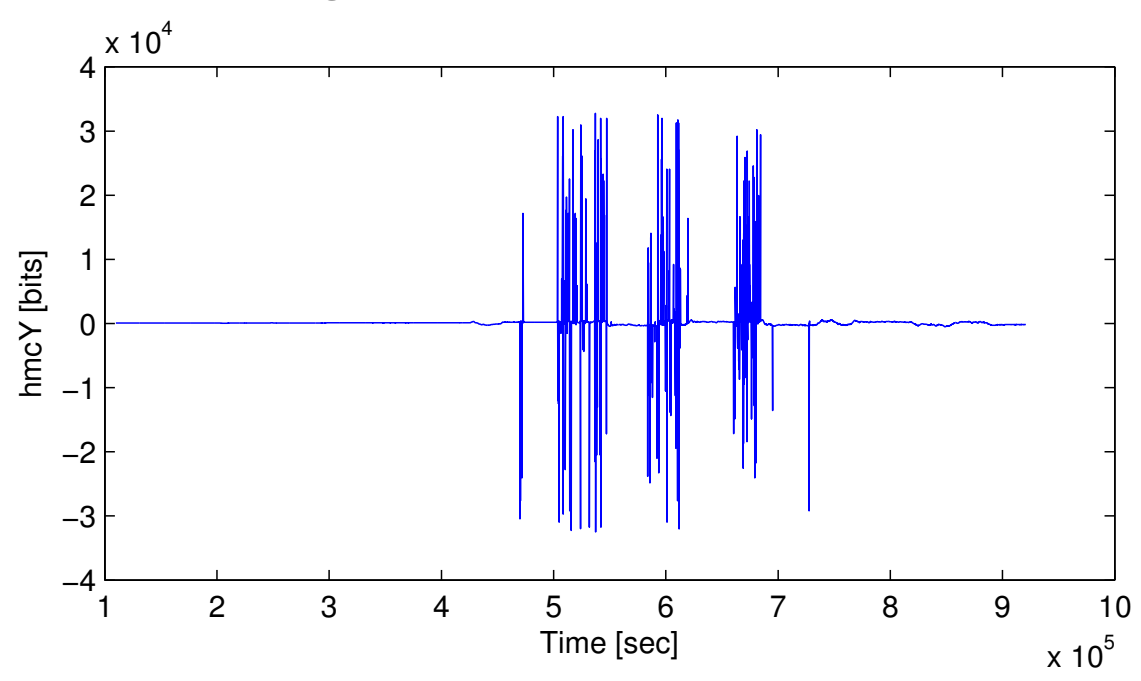

Figure E.13: press0 vs. Time

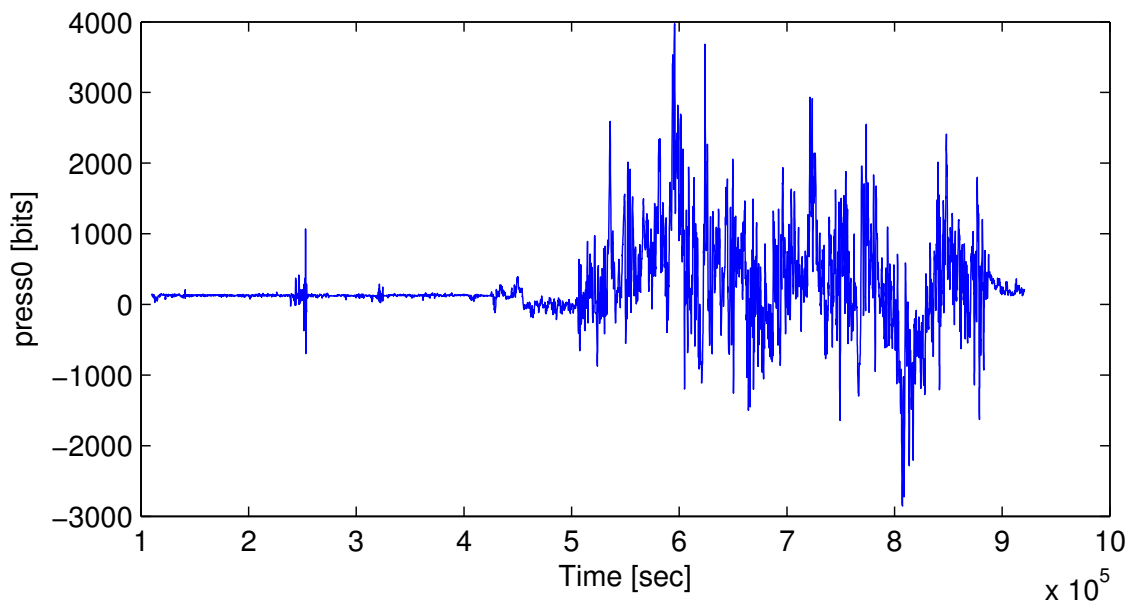

Figure E.14: press1 vs. Time

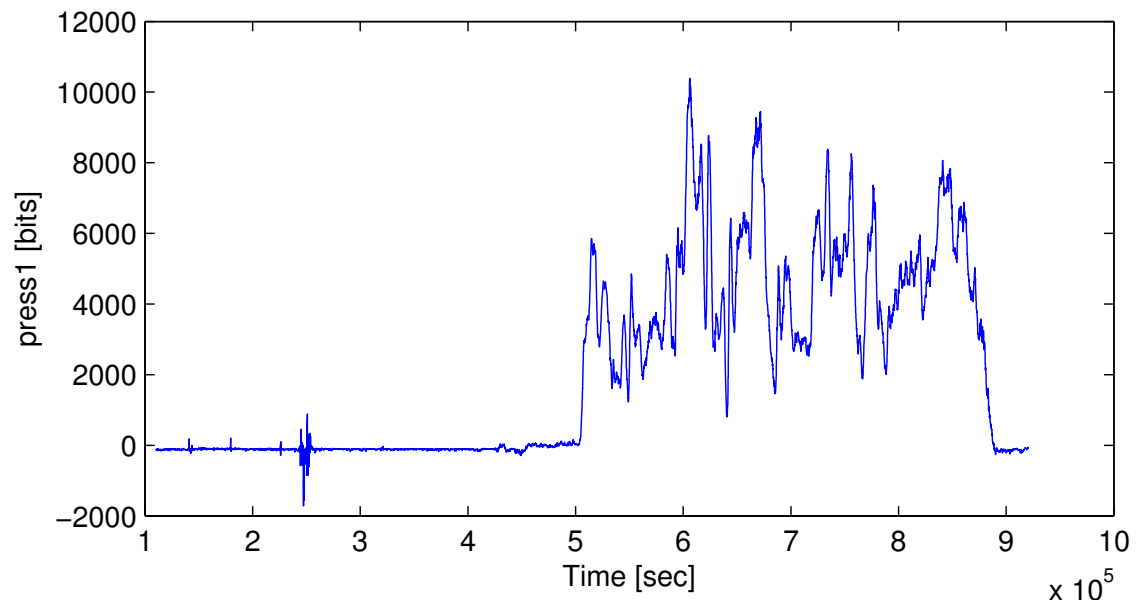


Figure E.15: press2 vs. Time

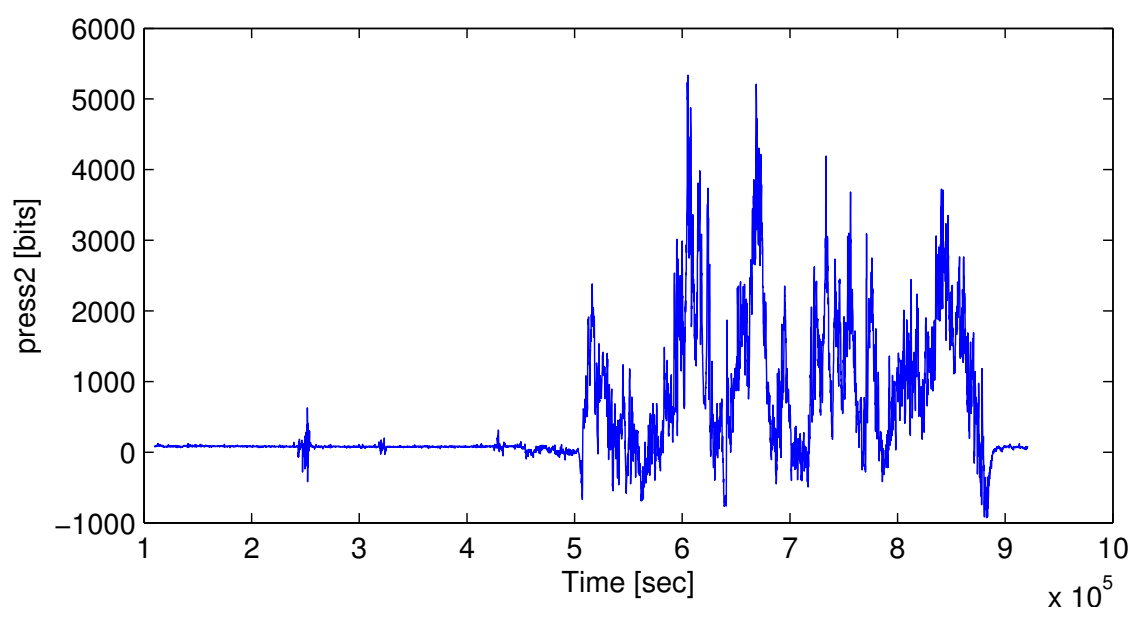

Figure E.16: press3 vs. Time

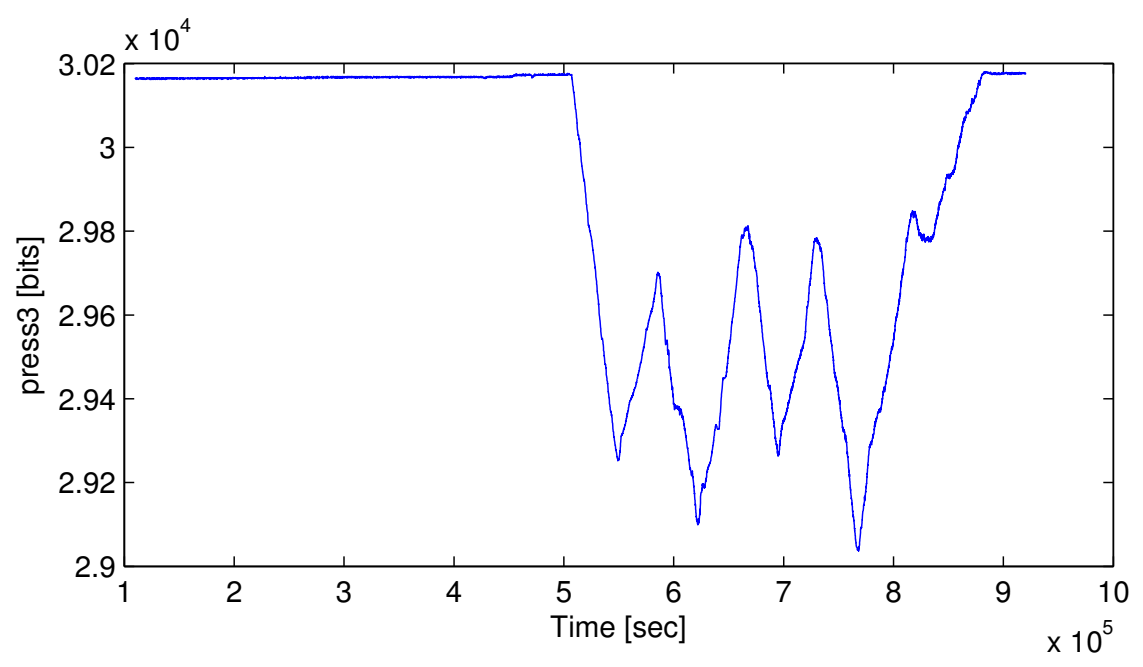


Figure E.17: gpsLat vs. Time

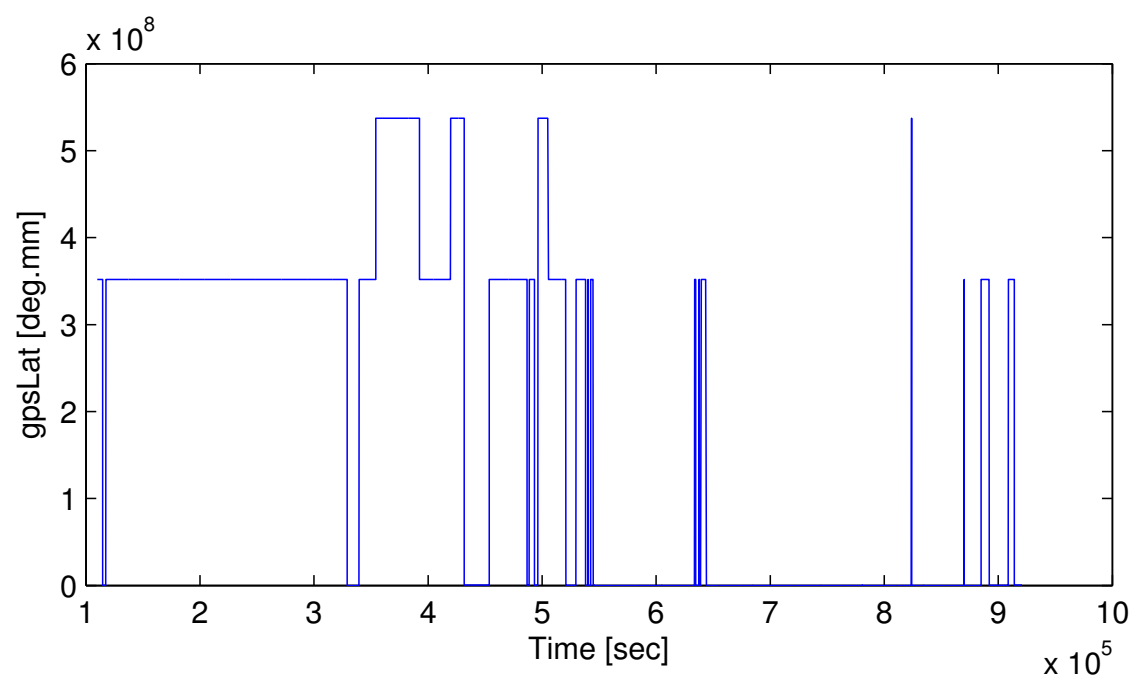

Figure E.18: gpsLong vs. Time

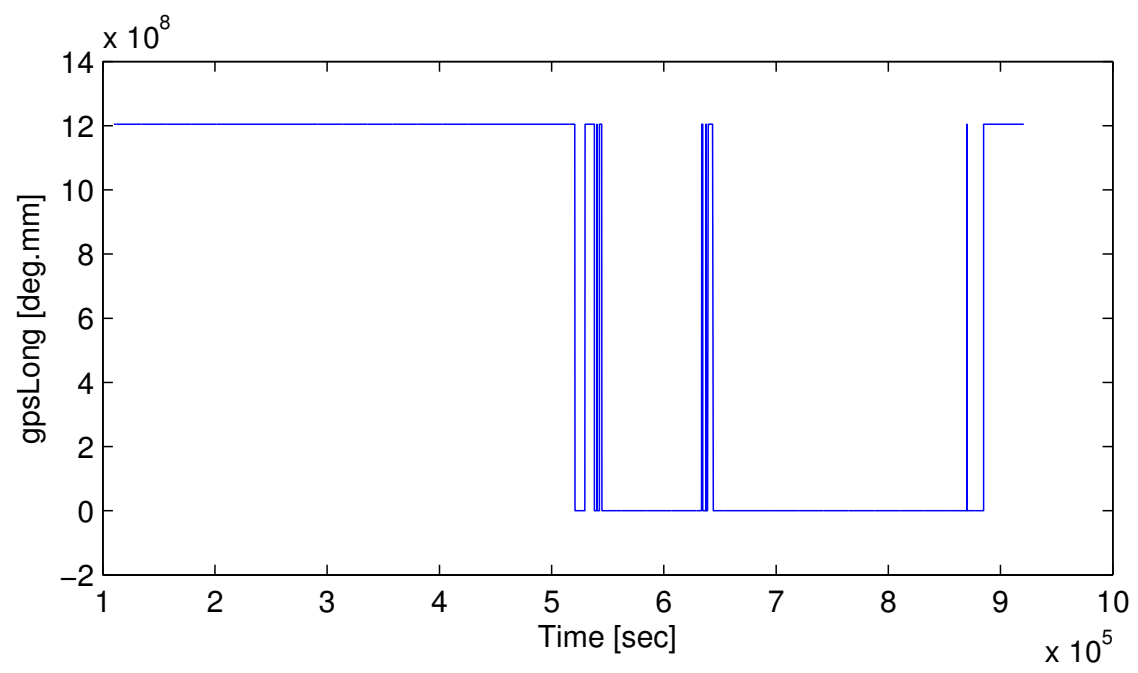


Figure E.19: gpsSpd vs. Time

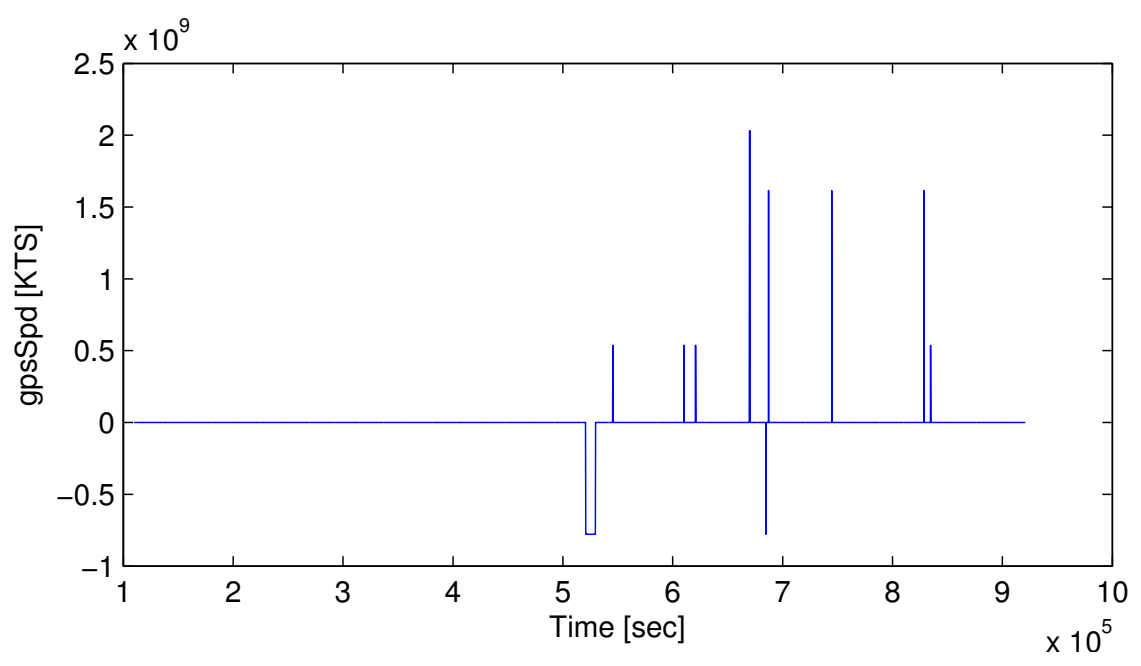

Figure E.20: gpsCrs vs. Time

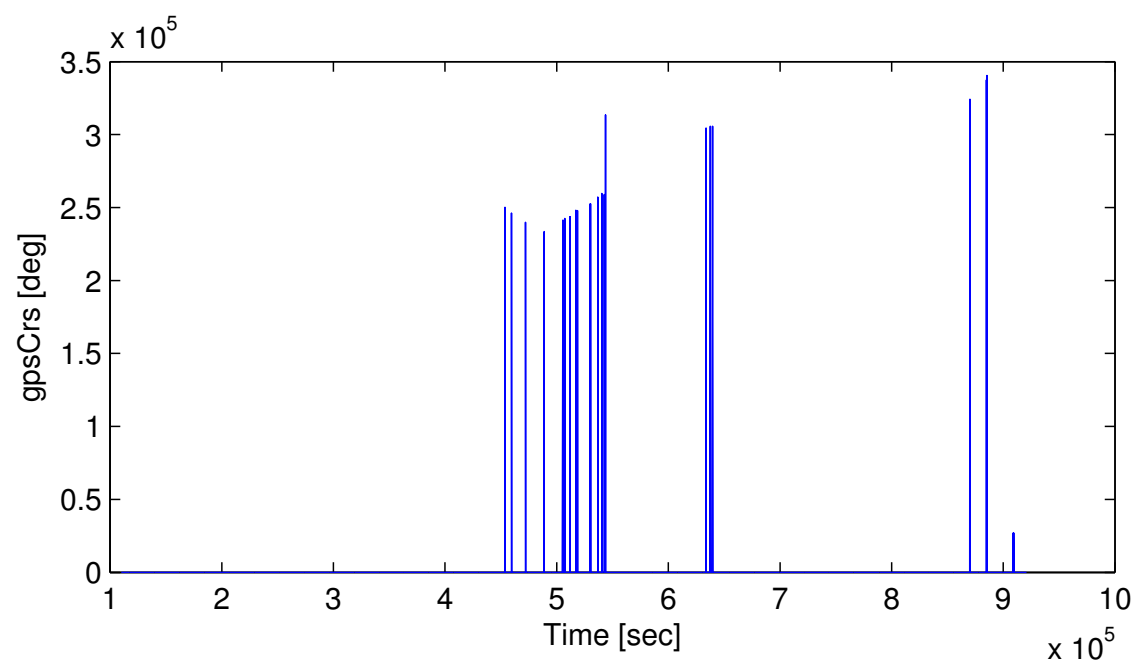


Figure E.21: date vs. Time

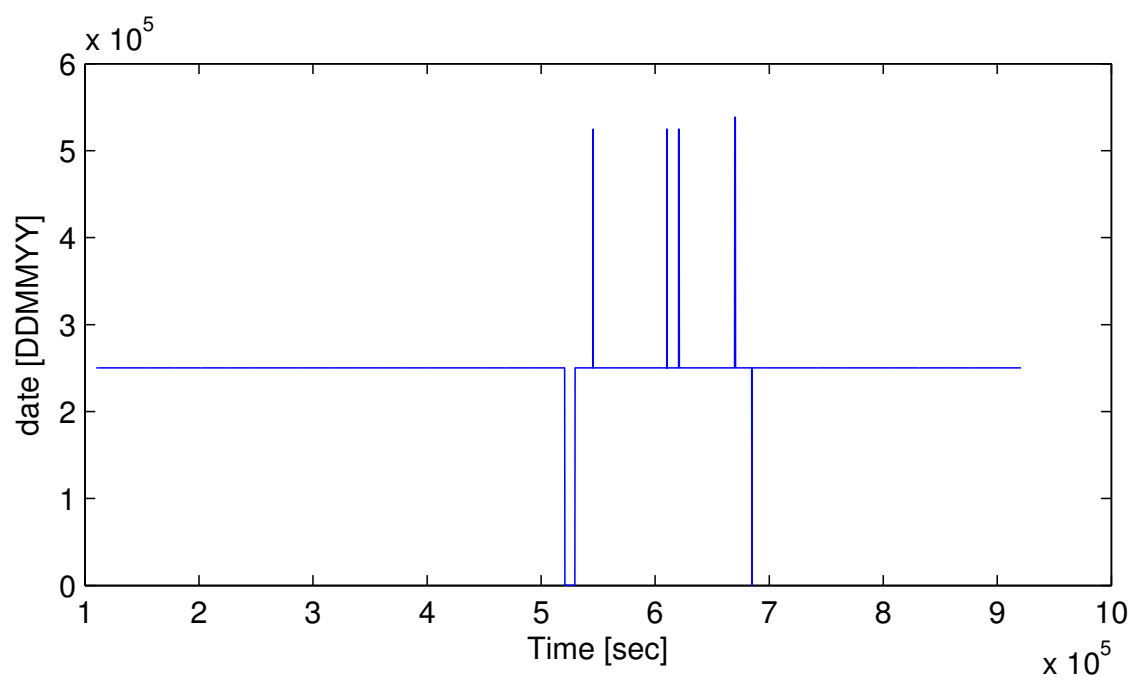

Figure E.22: CS vs. Time

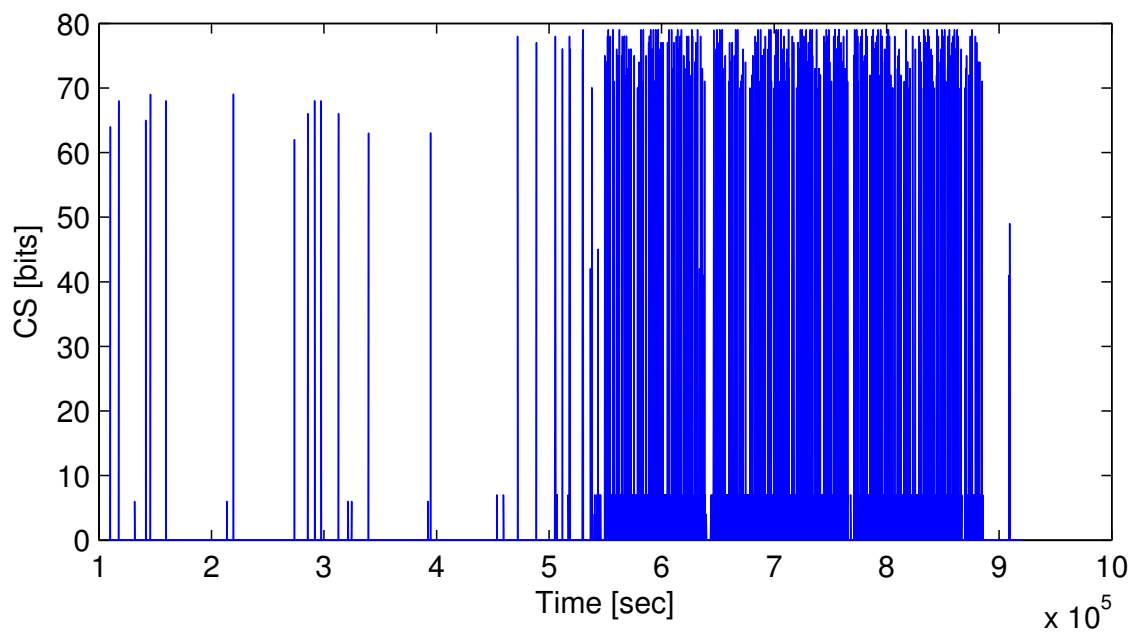


Figure E.23: temperature vs. Time

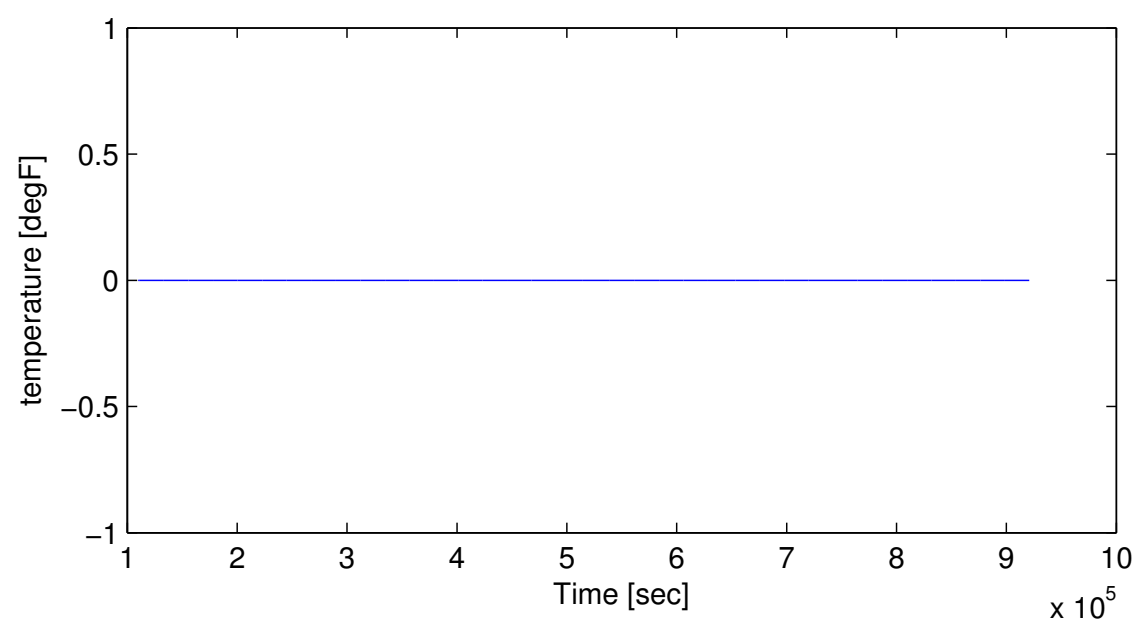

Figure E.24: pwm0 vs. Time

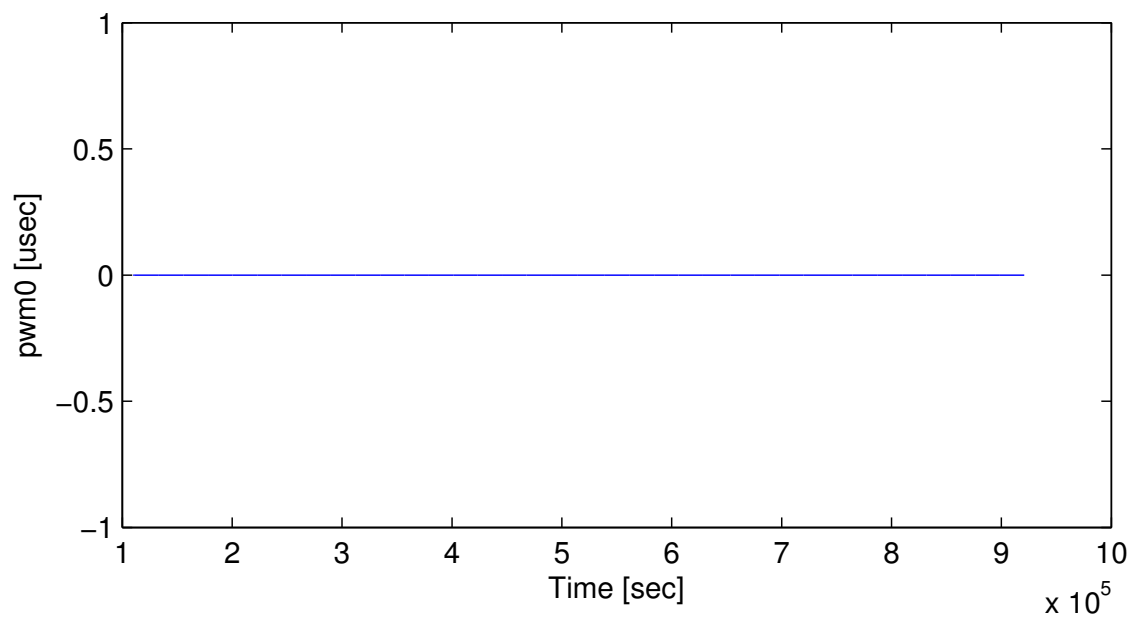


Figure E.25: pwm1 vs. Time

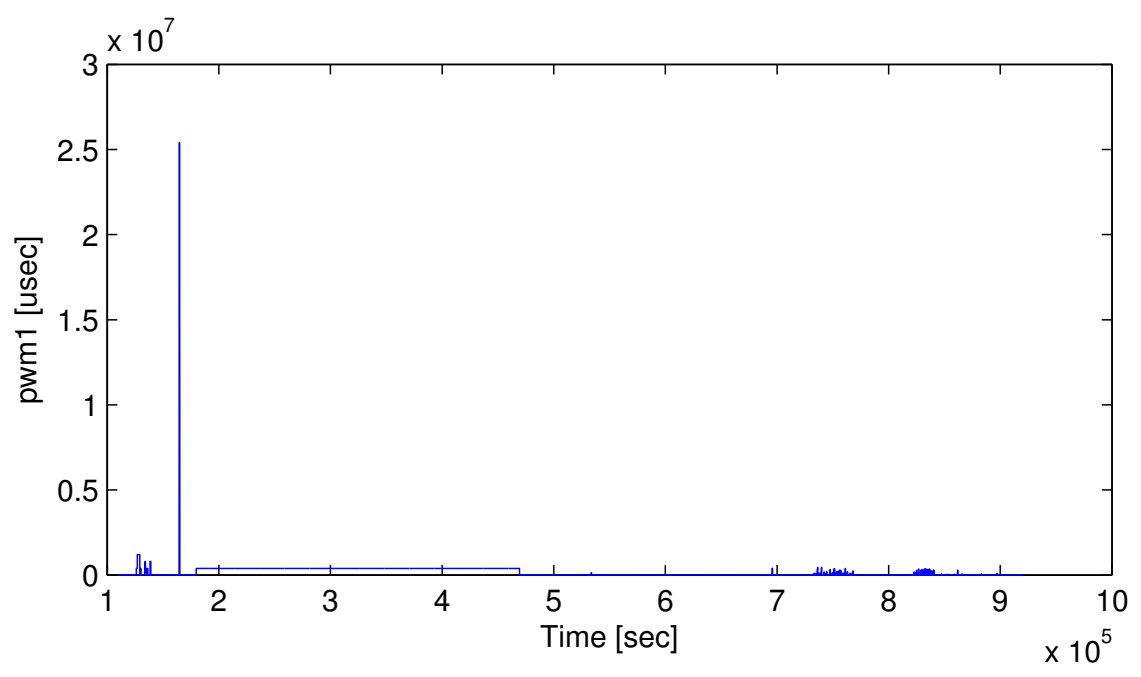

Figure E.26: pwm2 vs. Time

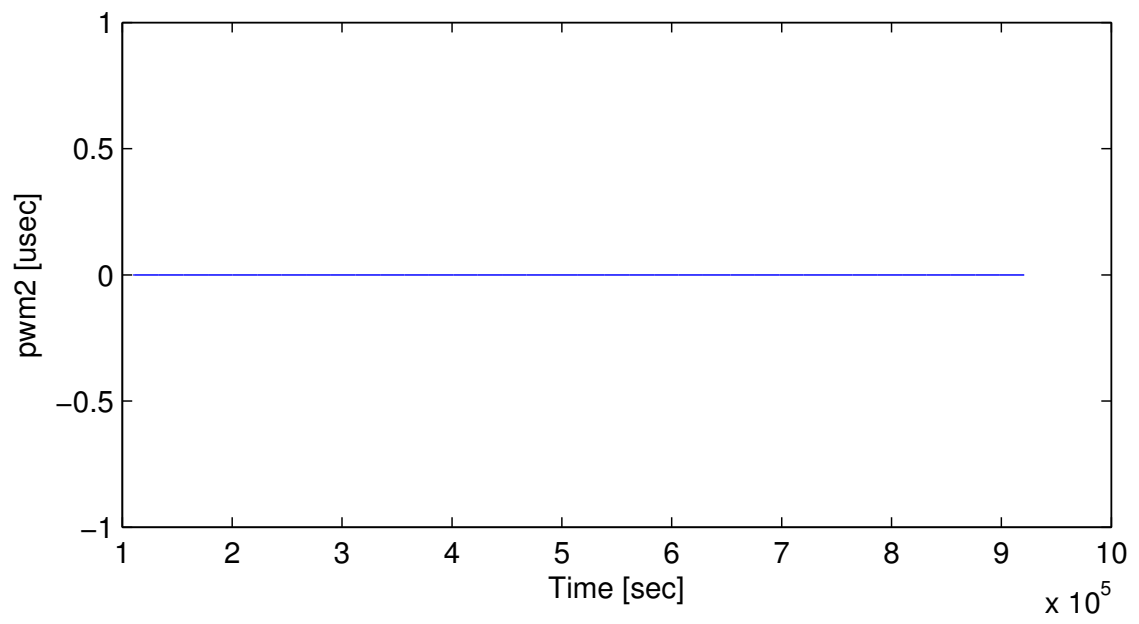


Figure E.27: pwm3 vs. Time

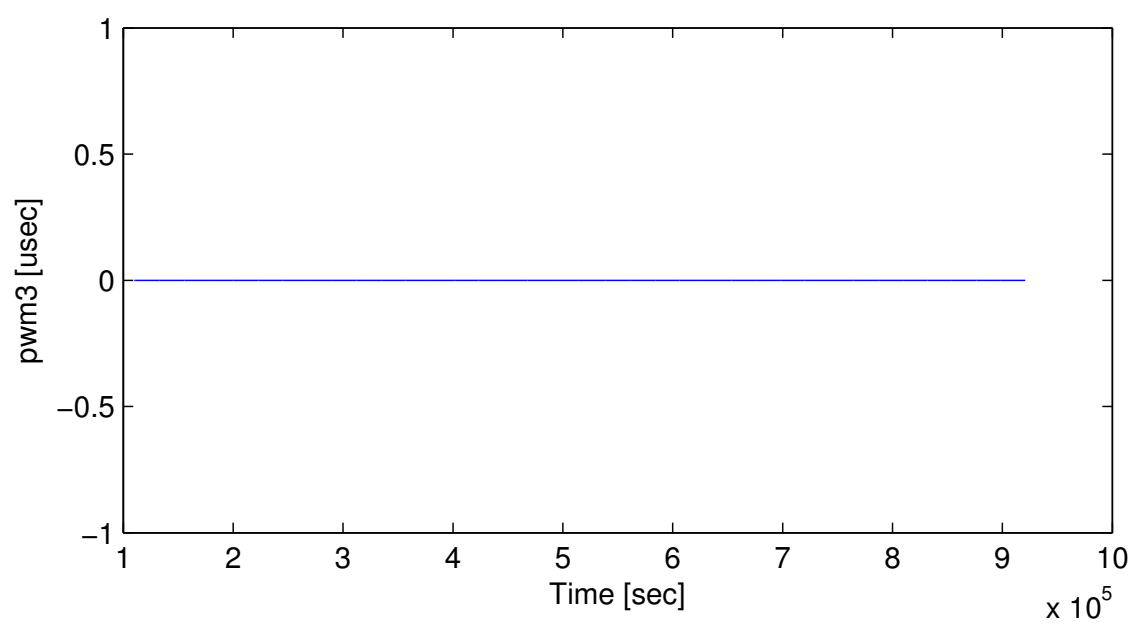

Figure E.28: pwm4 vs. Time

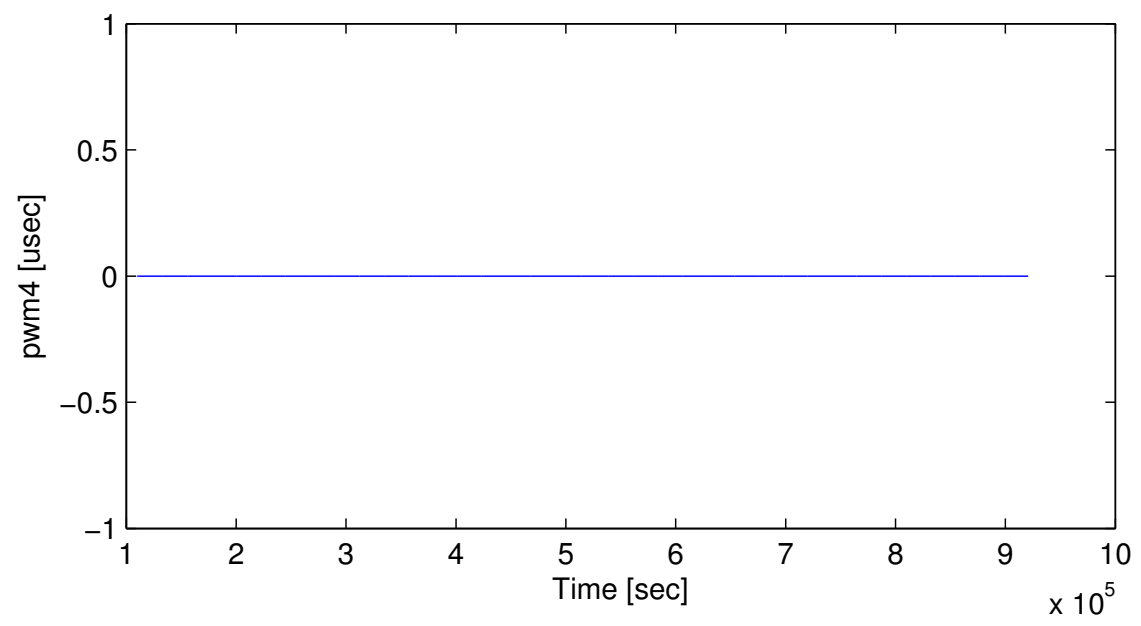

Figure E.29: pwm5 vs. Time

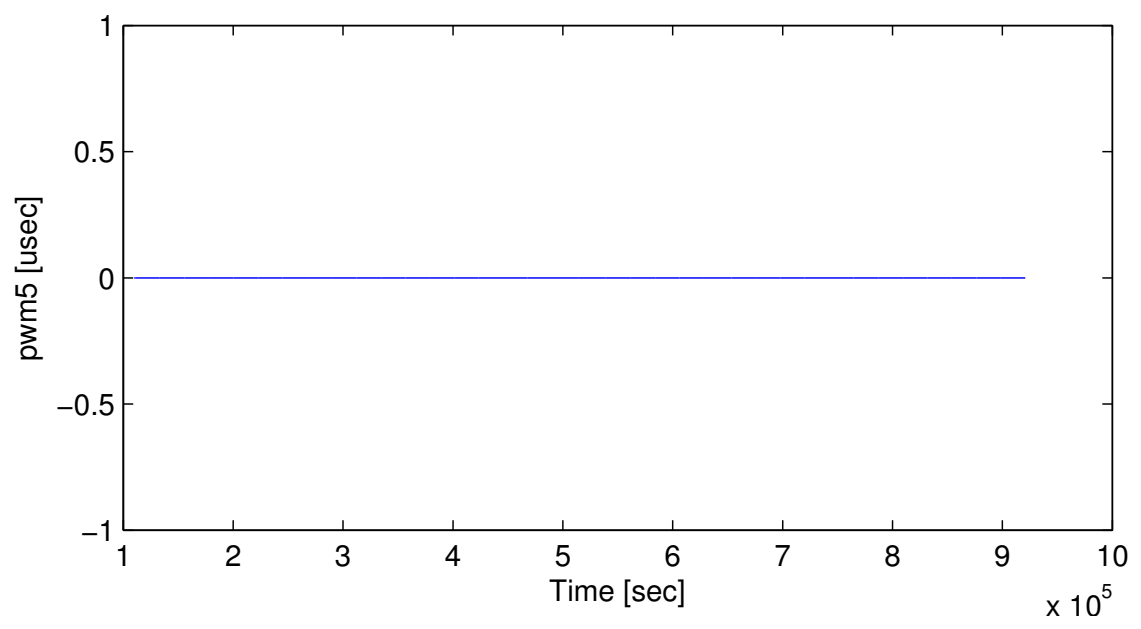


Figure E.30: pwm6 vs. Time

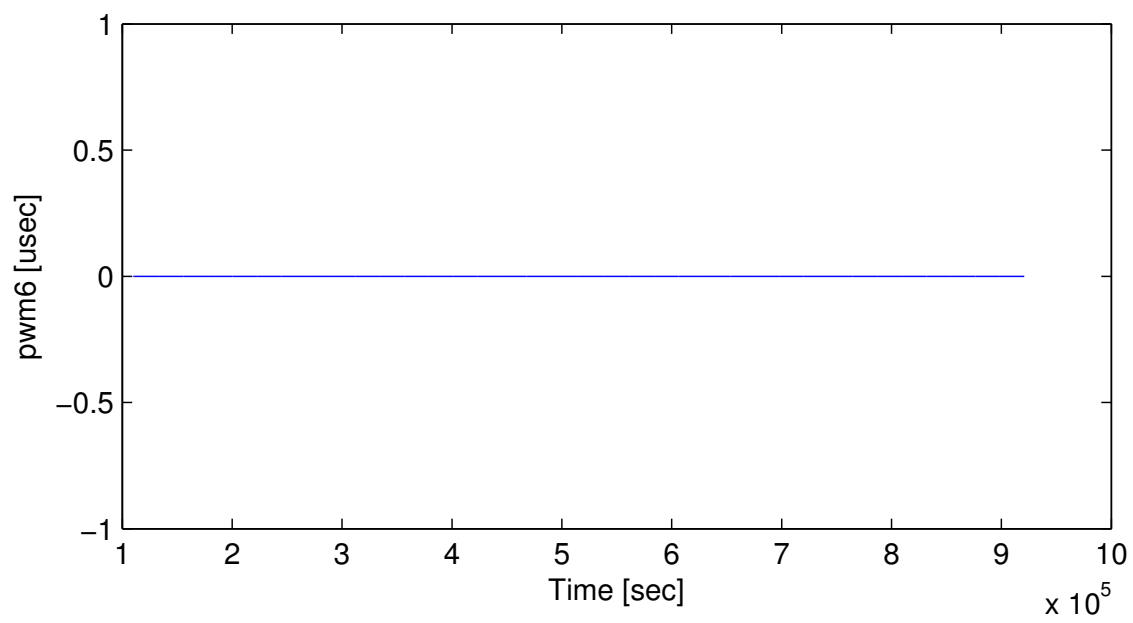

Figure E.31: pwm7 vs. Time

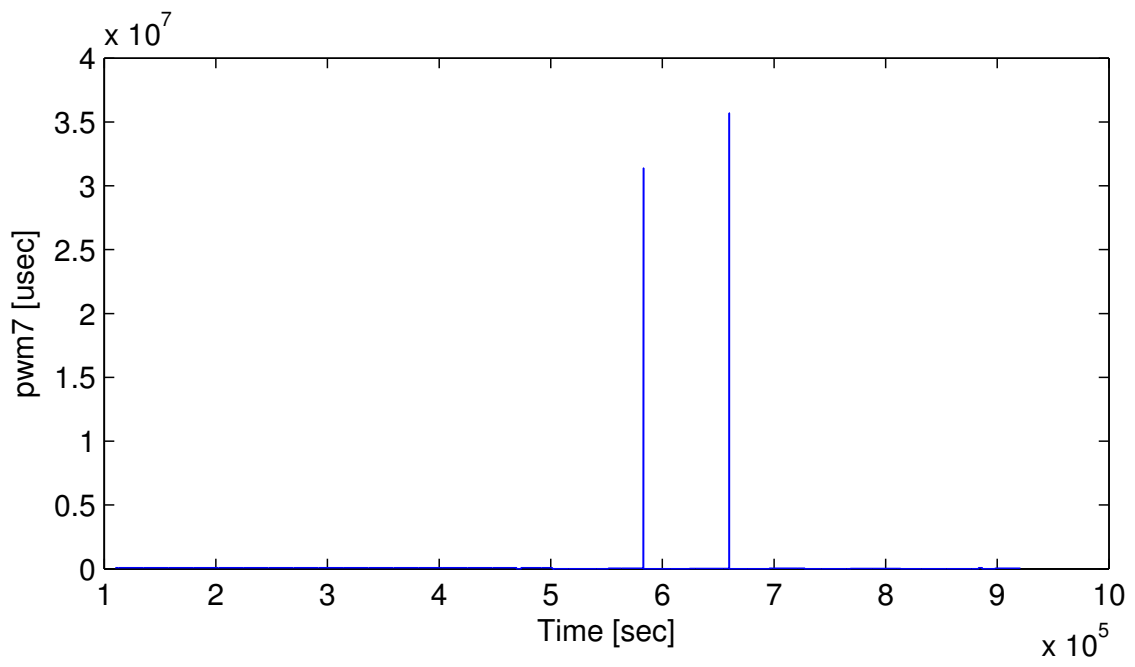


Figure E.32: deltaT vs. Time

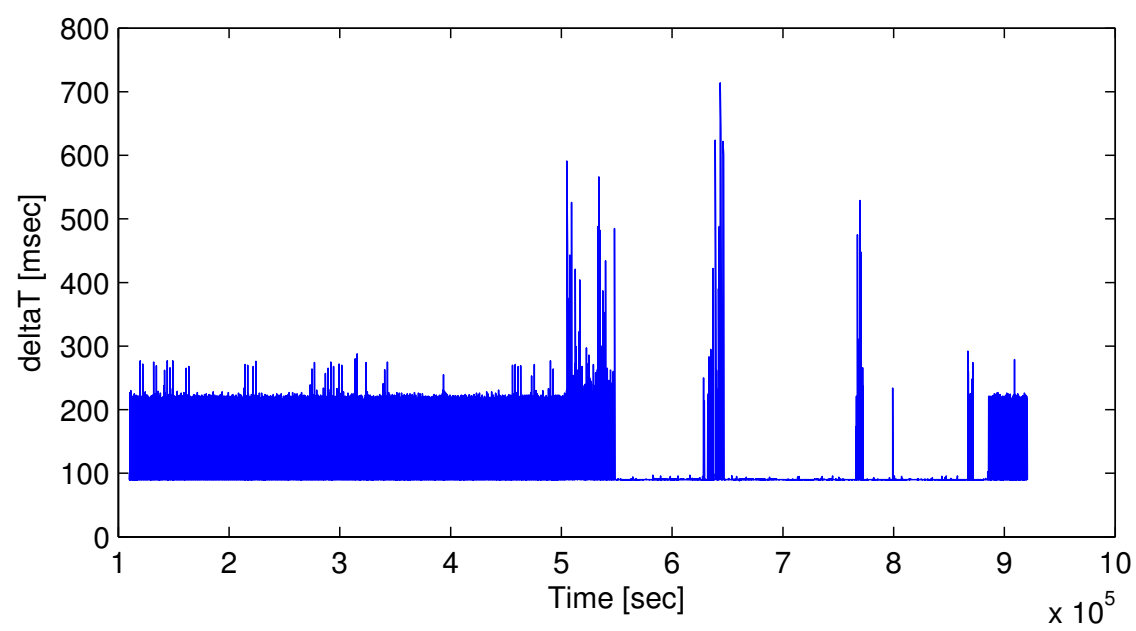

\section{E.2 Sample System Ouput - Units Data}

Figure E.33: accelX vs. Time

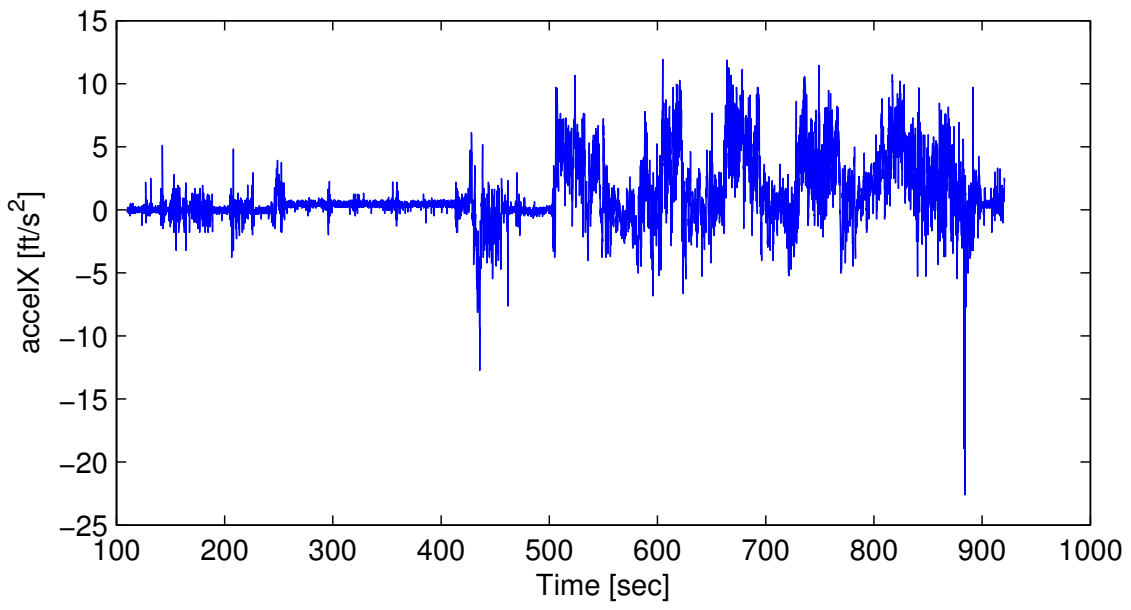


Figure E.34: accelY vs. Time

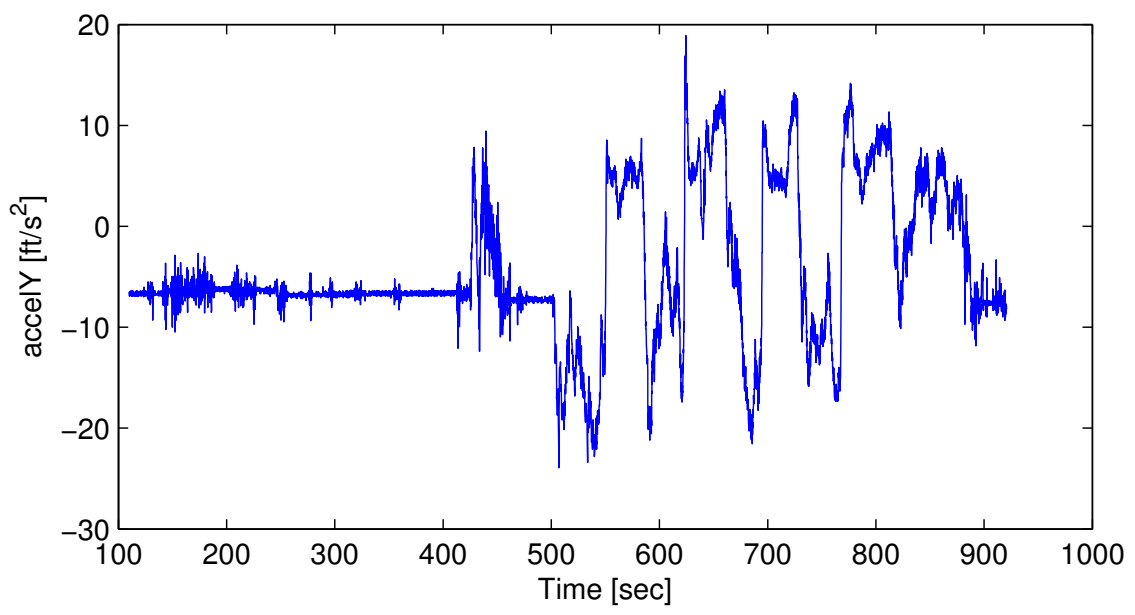

Figure E.35: accelZ vs. Time

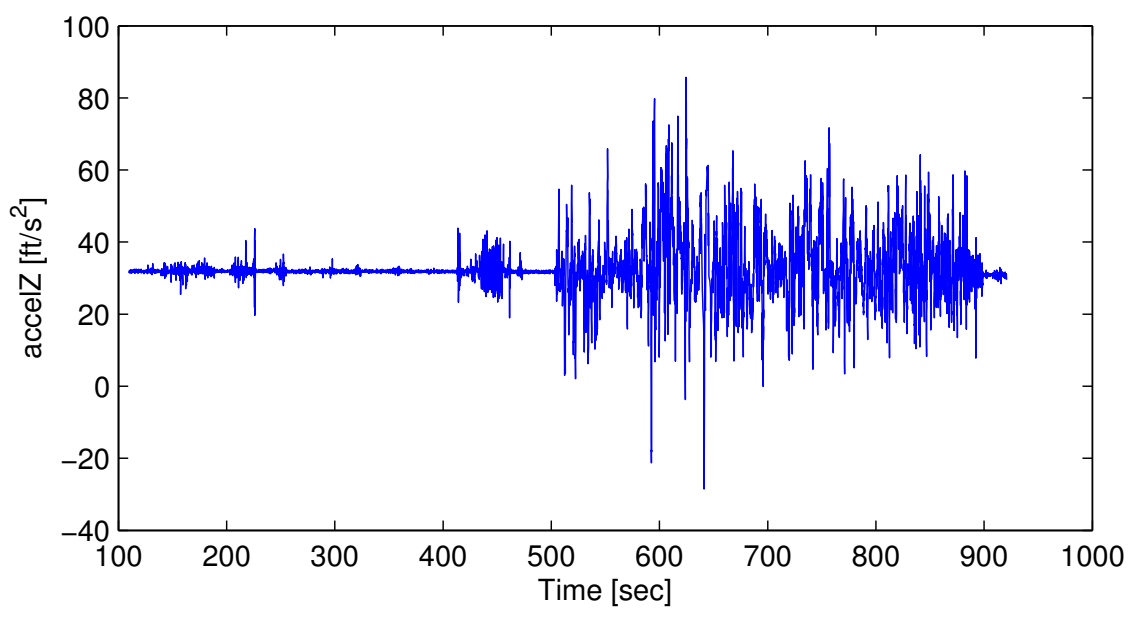

Figure E.36: gyroX vs. Time

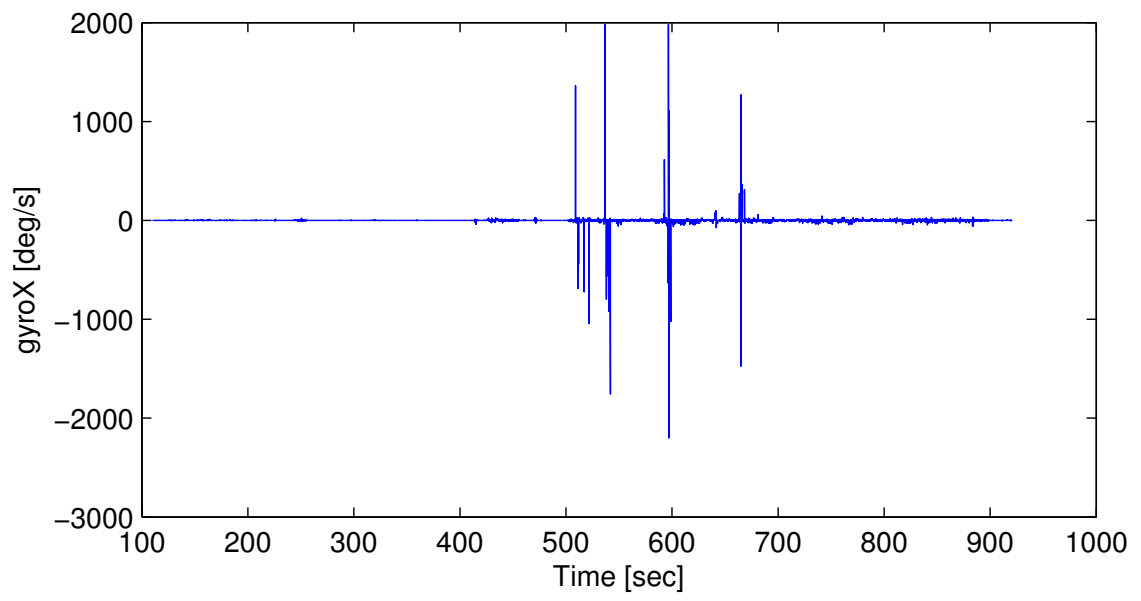


Figure E.37: gyroY vs. Time

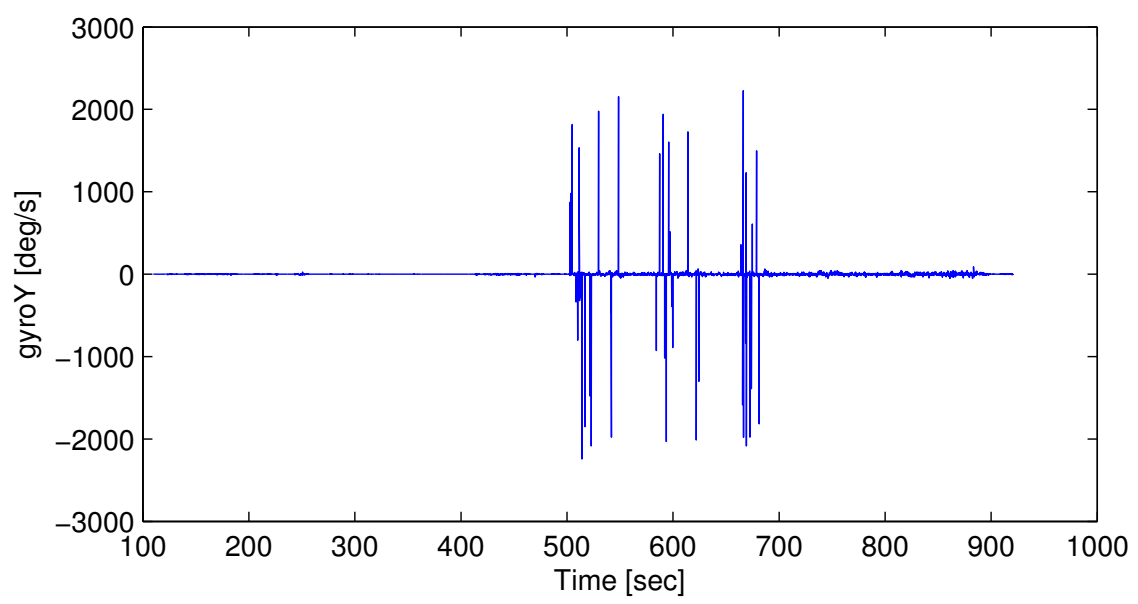

Figure E.38: gyroZ vs. Time

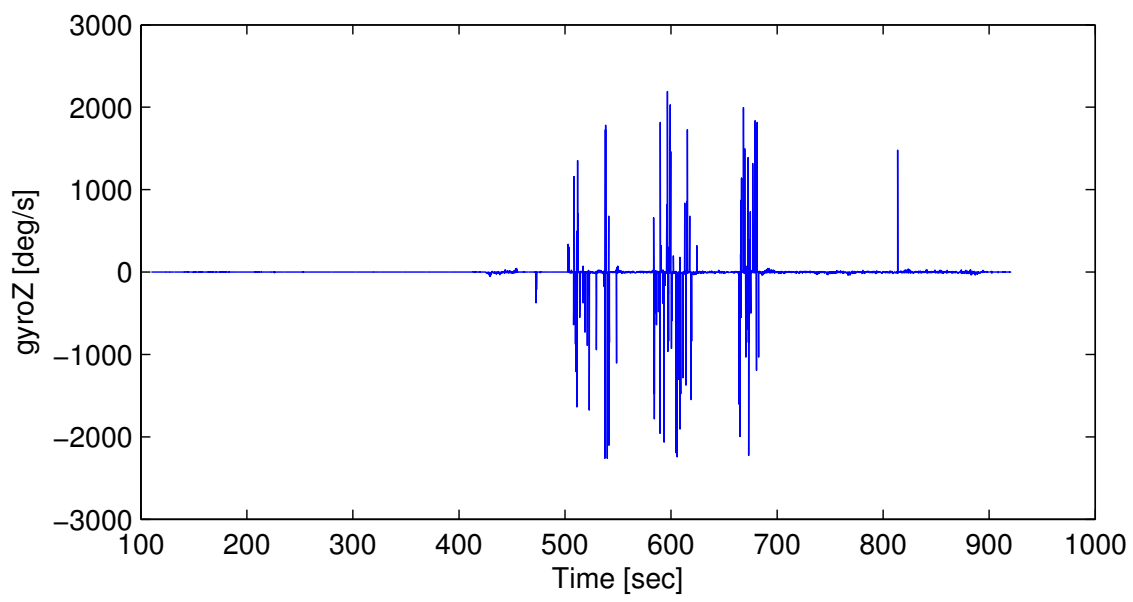

Figure E.39: magX vs. Time

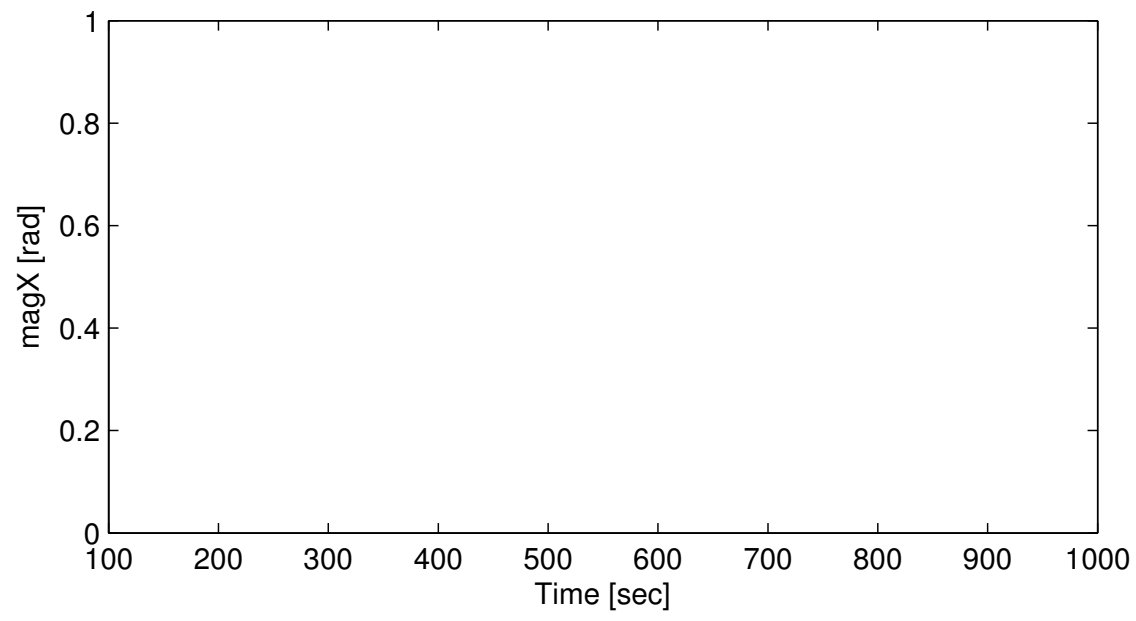


Figure E.40: magY vs. Time

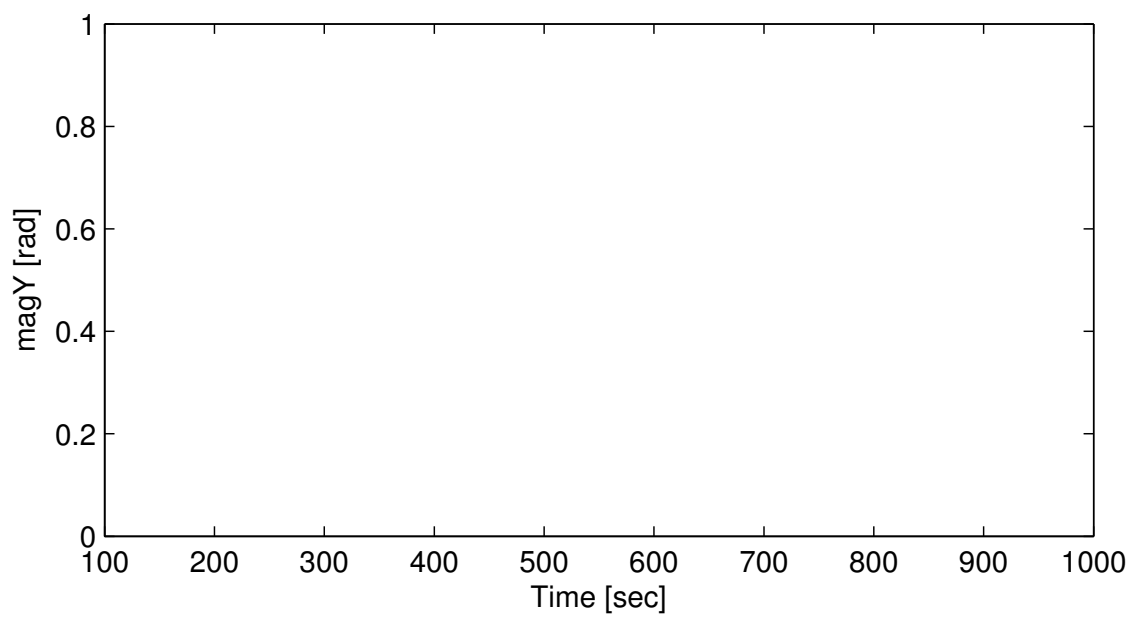

Figure E.41: magZ vs. Time

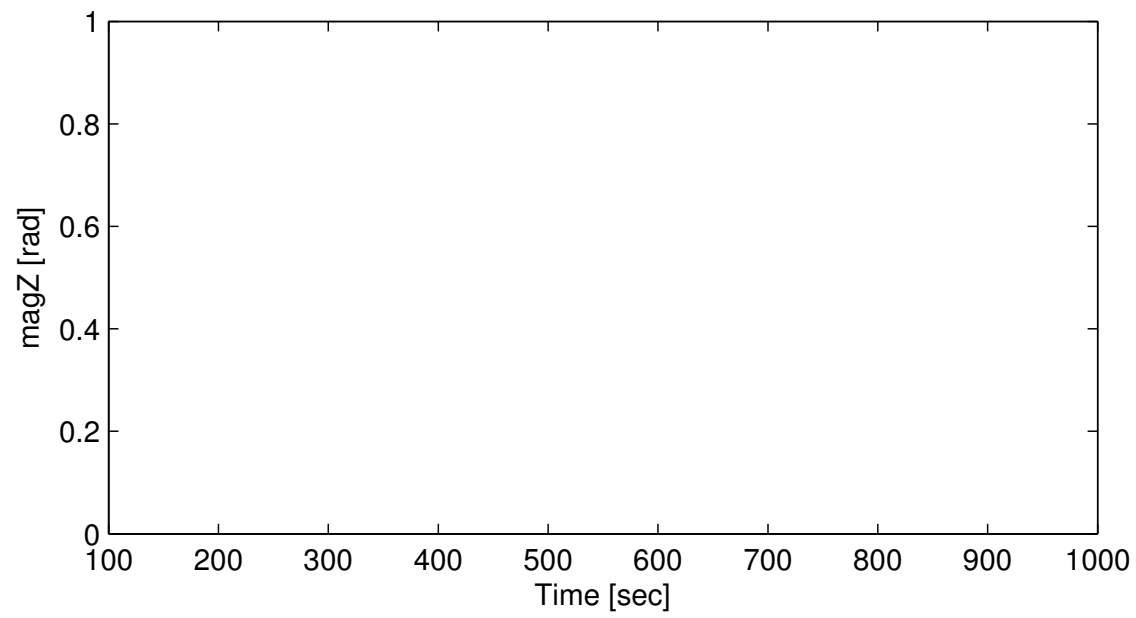

Figure E.42: hmcX vs. Time

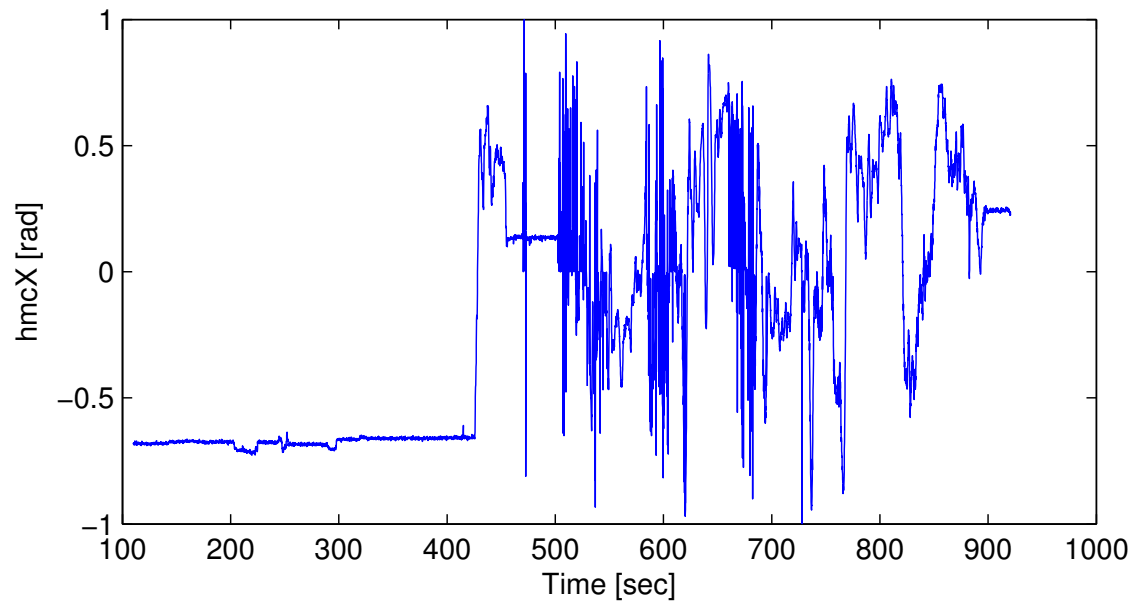


Figure E.43: hmcZ vs. Time

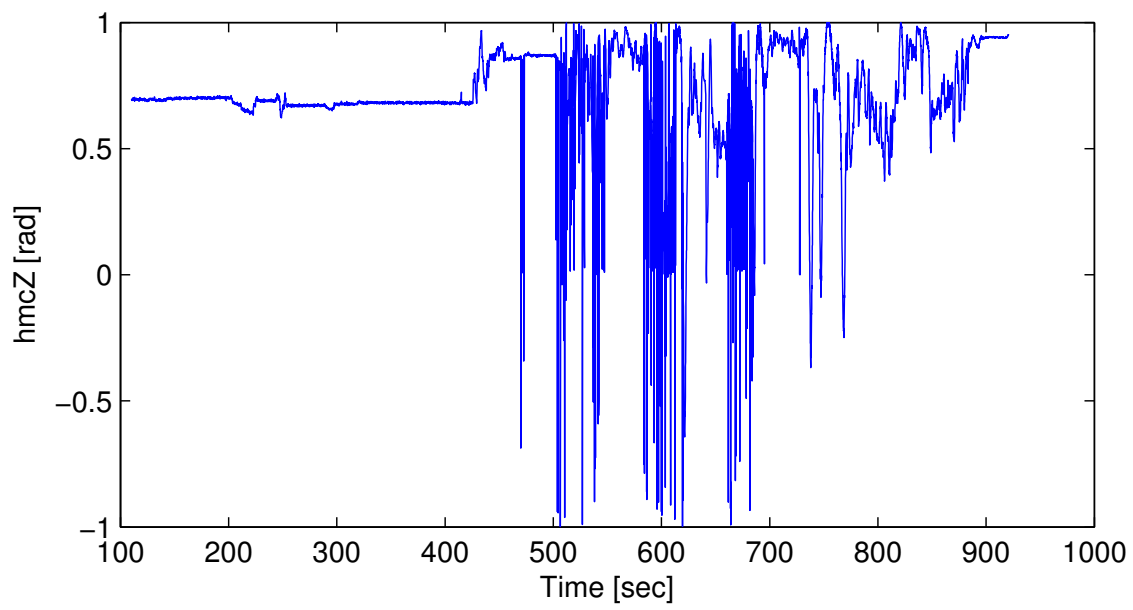

Figure E.44: hmcY vs. Time

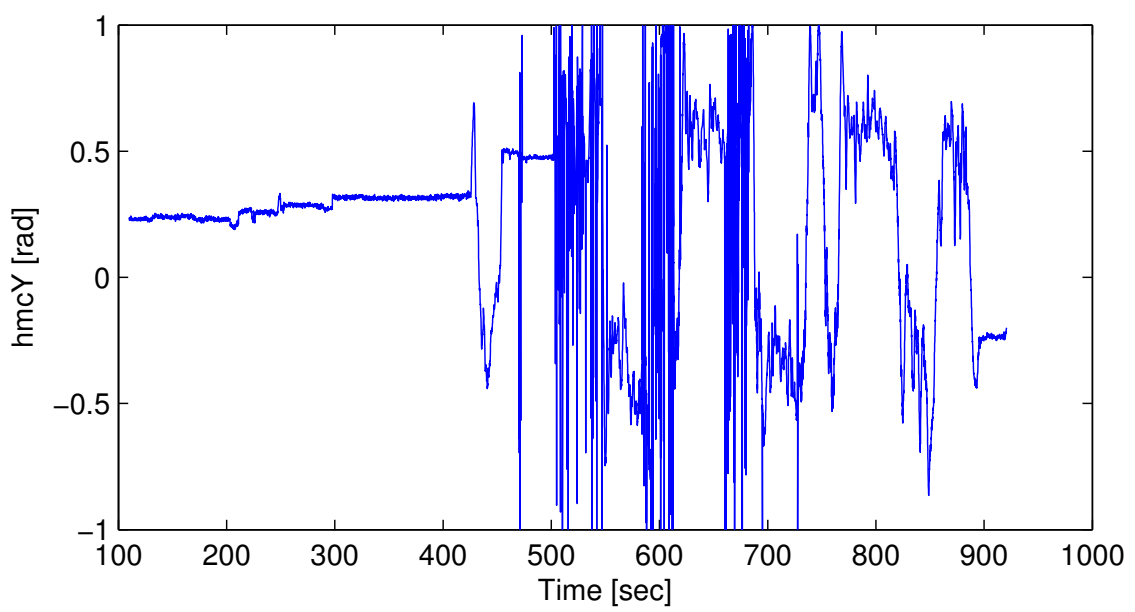

Figure E.45: press0 vs. Time

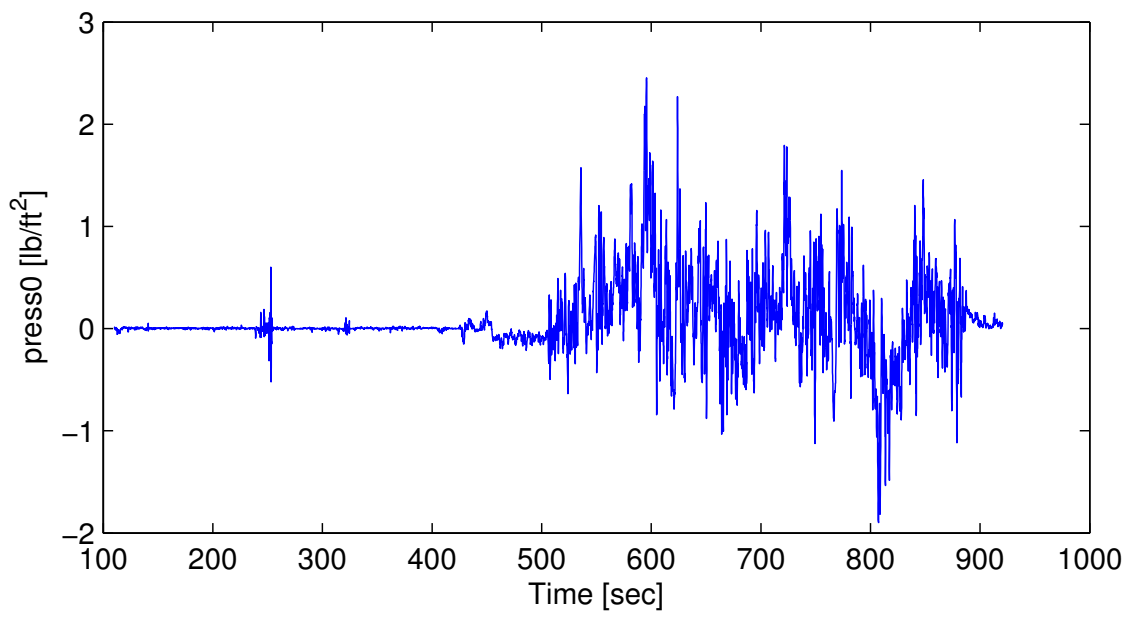


Figure E.46: press1 vs. Time

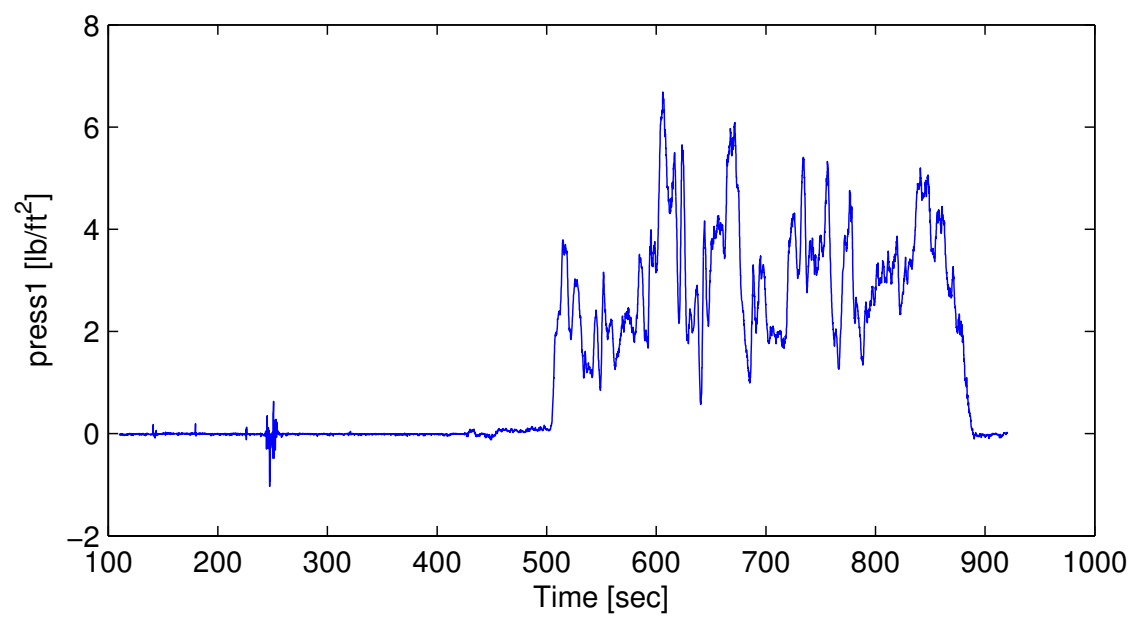

Figure E.47: press2 vs. Time

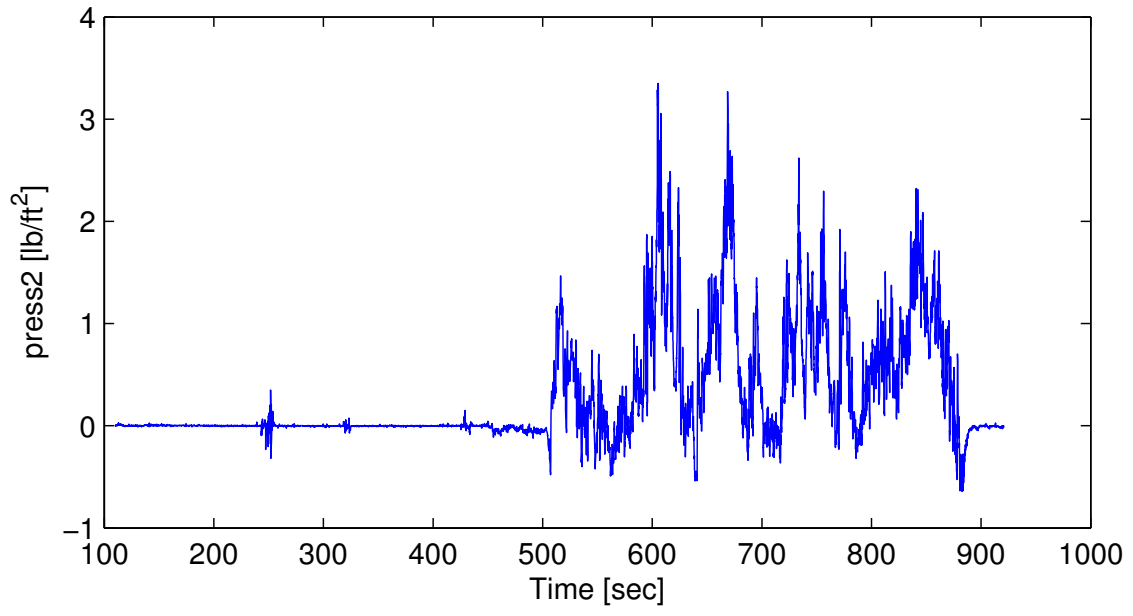

Figure E.48: press3 vs. Time

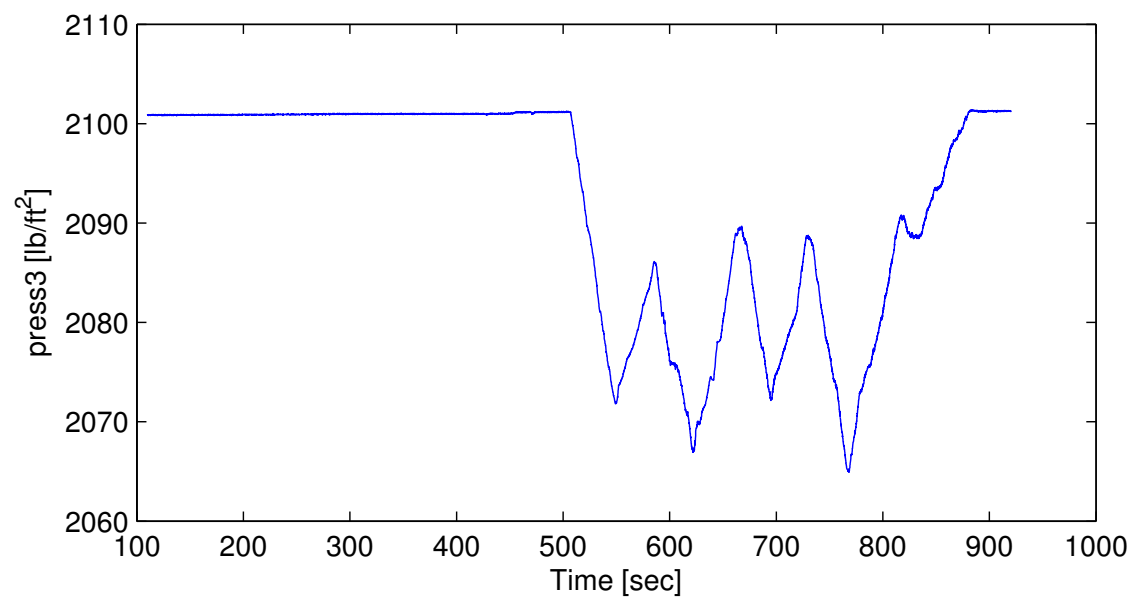


Figure E.49: gpsLat vs. Time

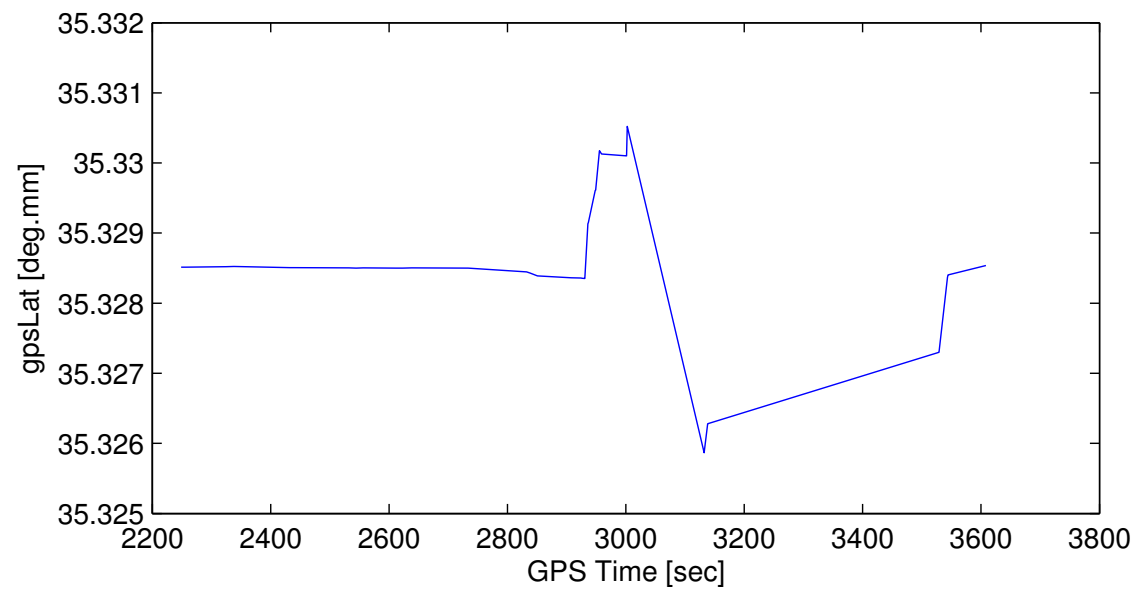

Figure E.50: gpsLong vs. Time

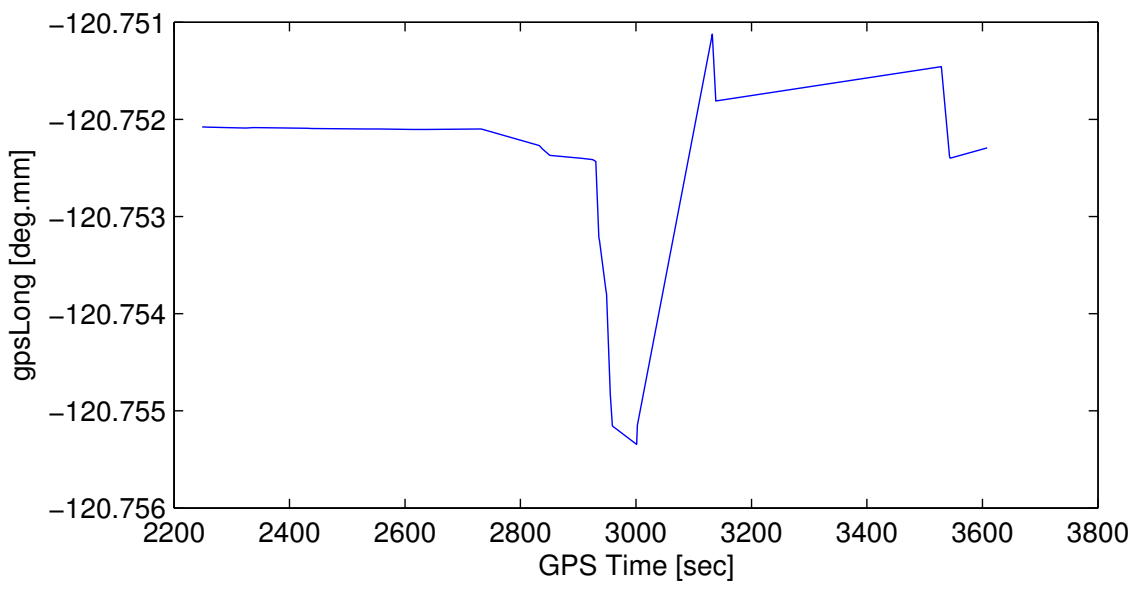

Figure E.51: gpsSpd vs. Time

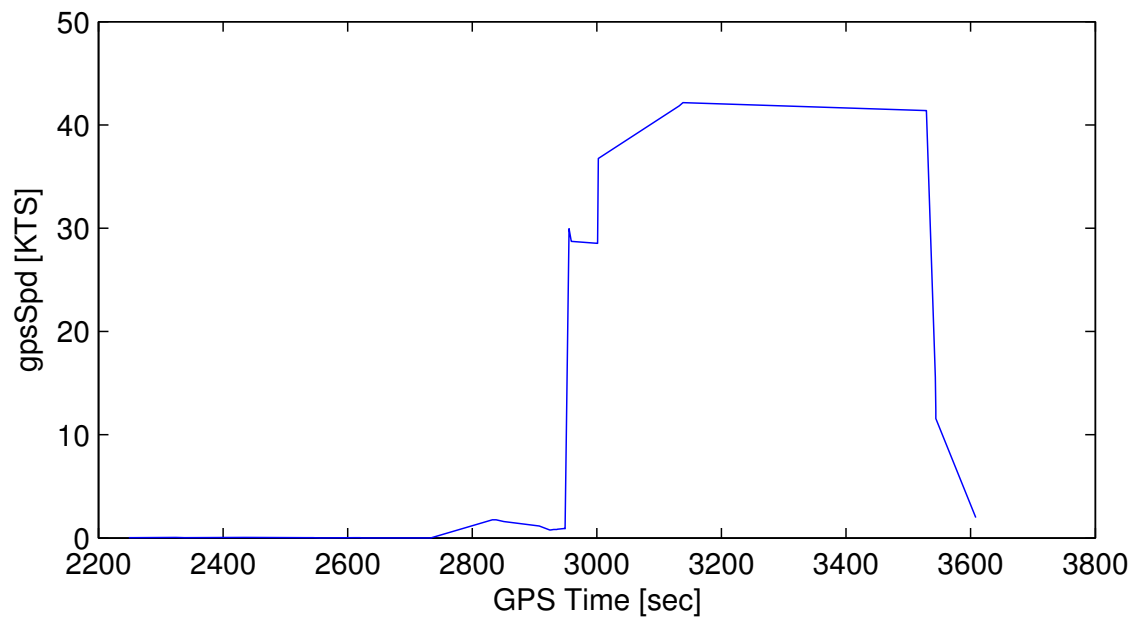


Figure E.52: gpsCrs vs. Time

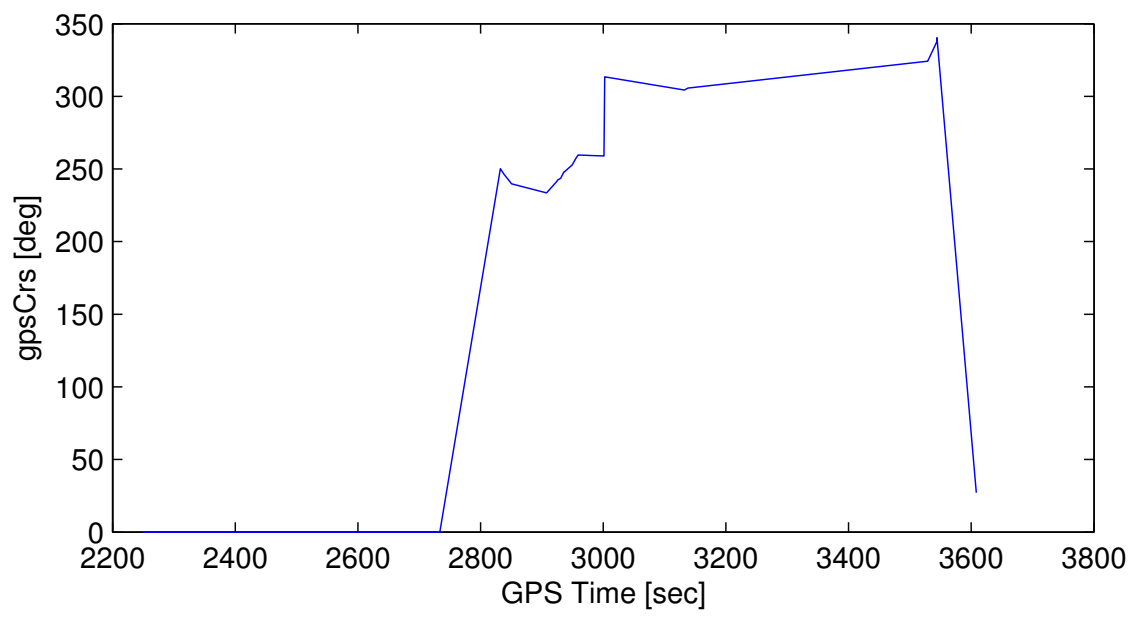

Figure E.53: date vs. Time

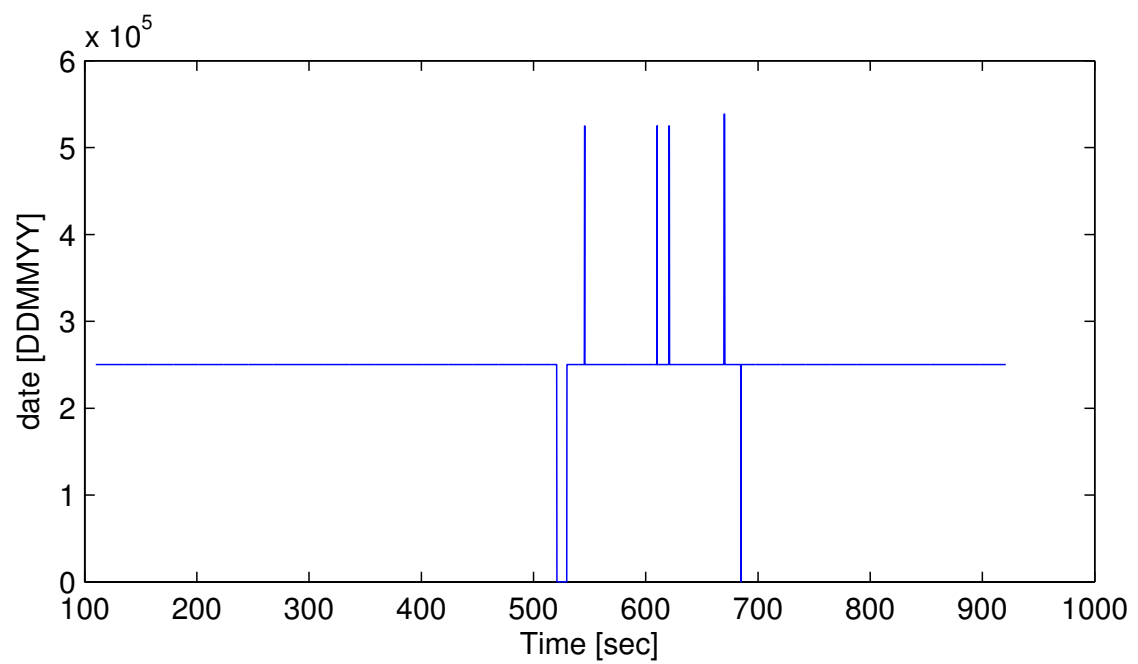


Figure E.54: CS vs. Time

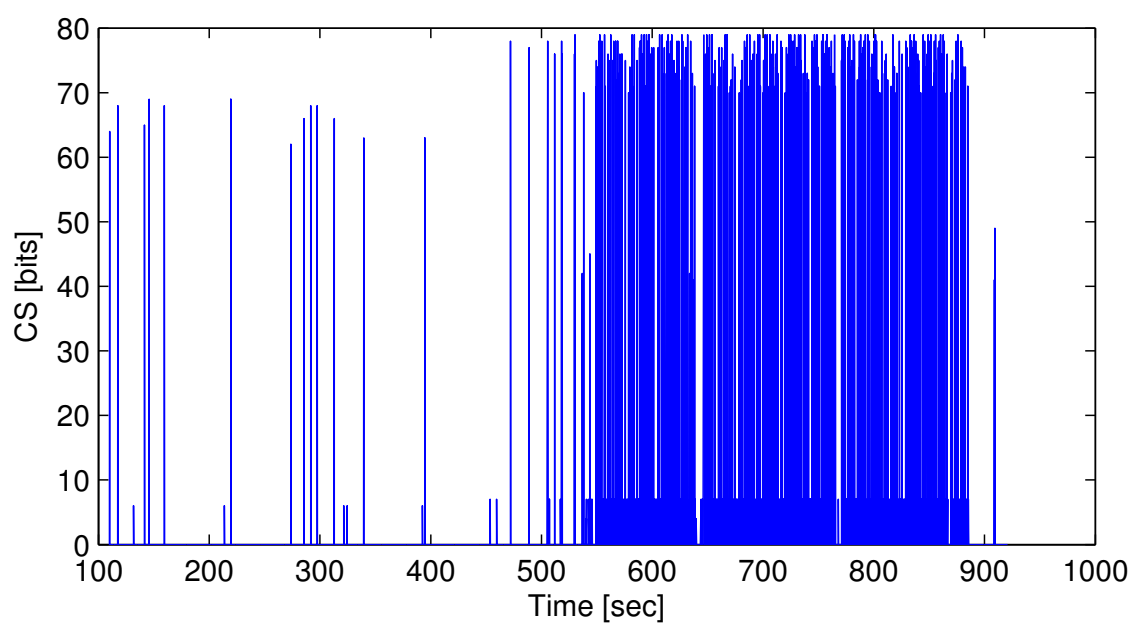

Figure E.55: temperature vs. Time

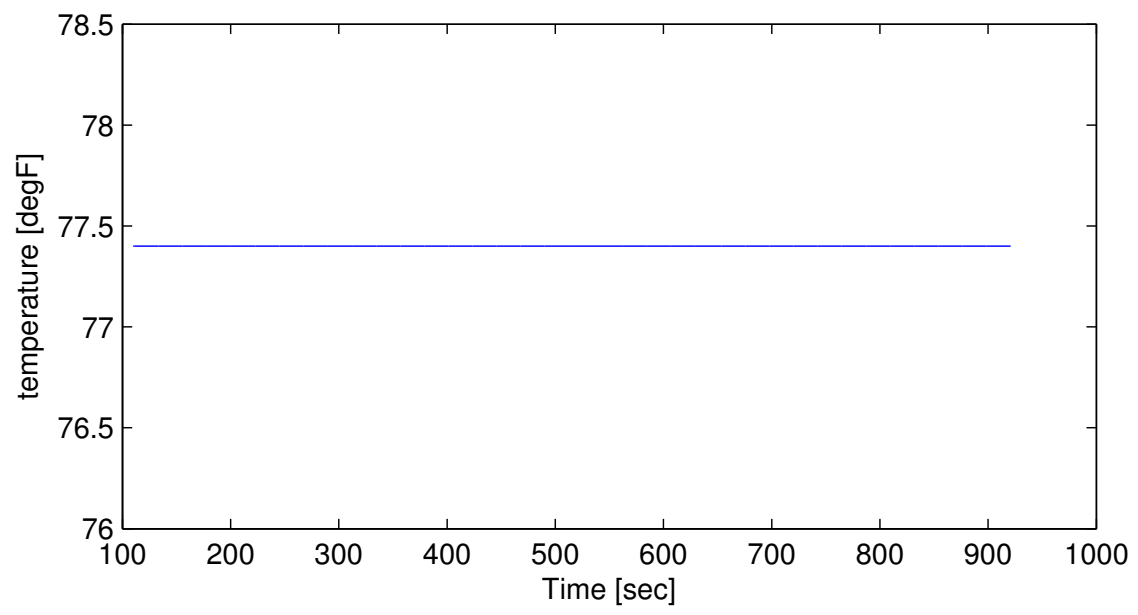

Figure E.56: pwm0 vs. Time

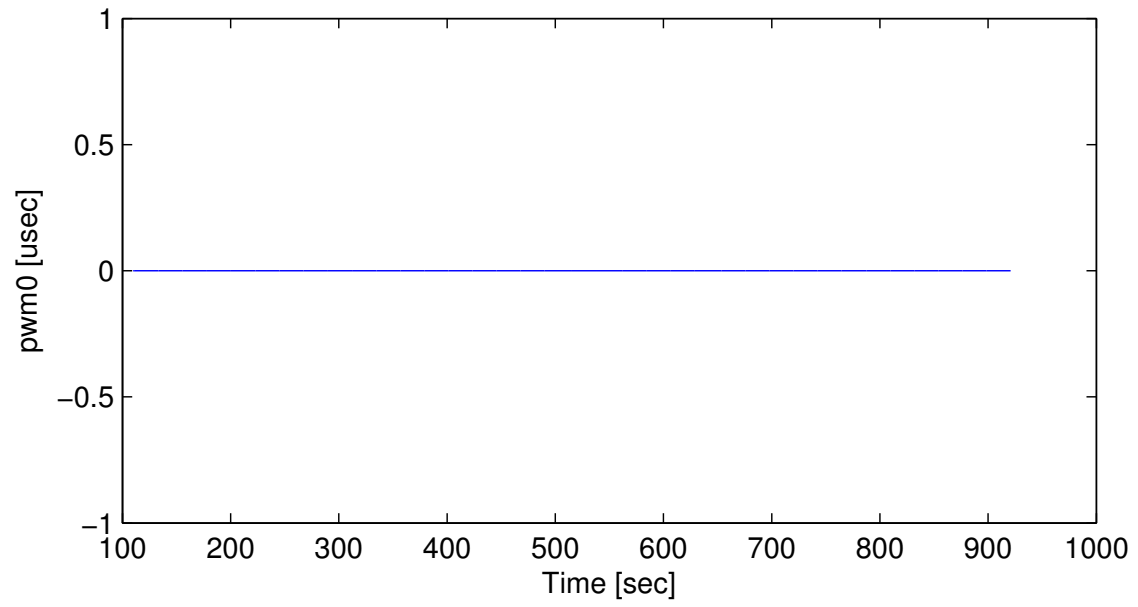


Figure E.57: pwm1 vs. Time

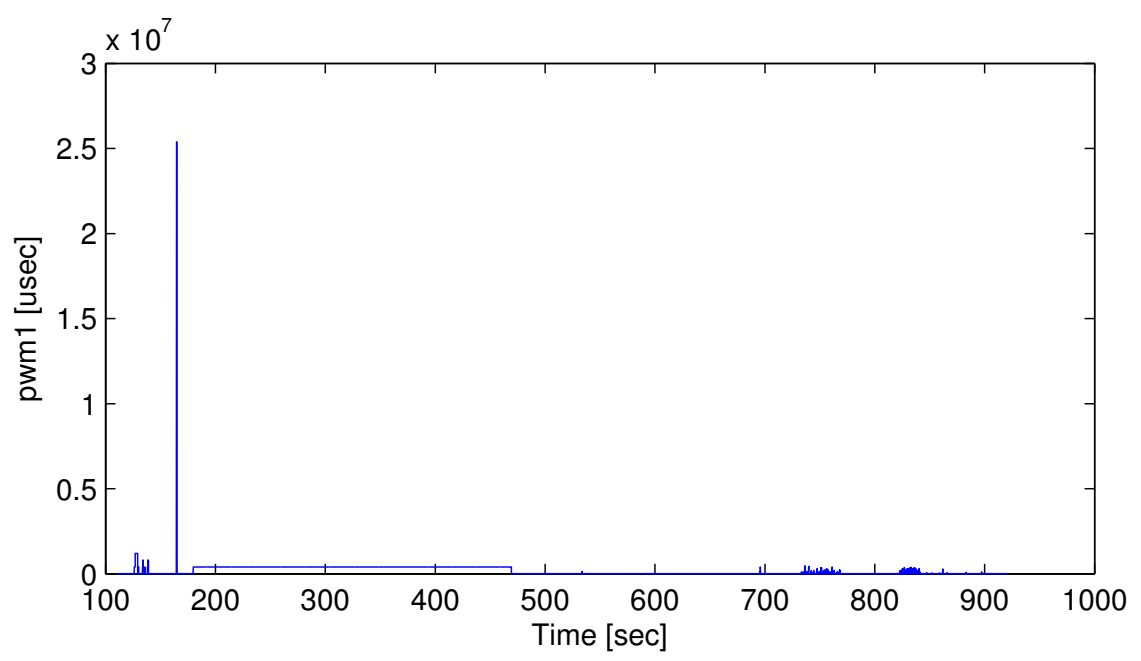

Figure E.58: pwm2 vs. Time

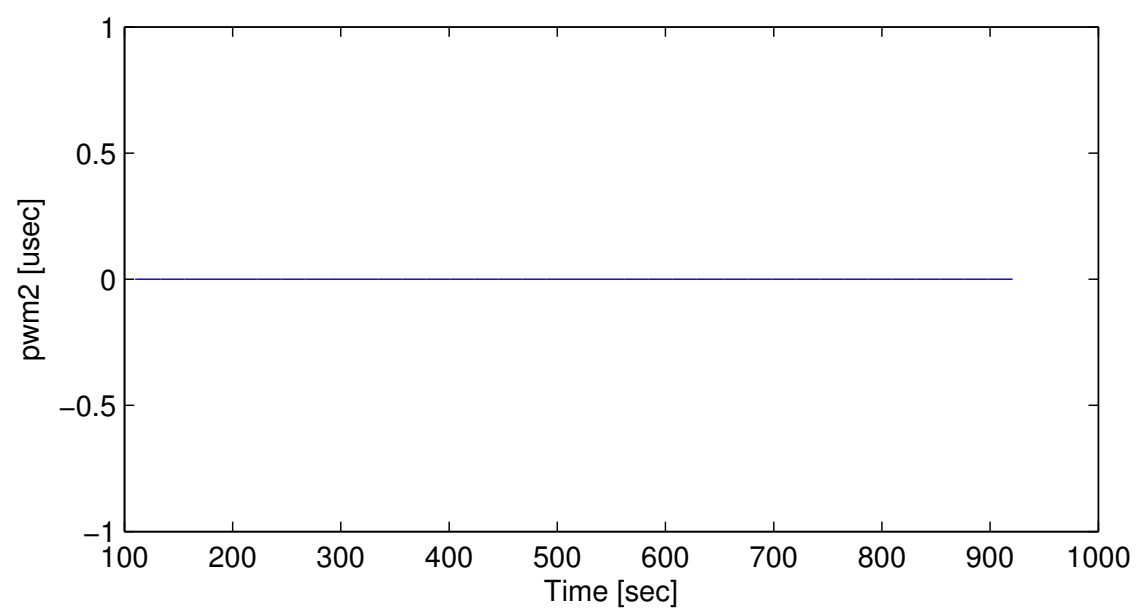

Figure E.59: pwm3 vs. Time

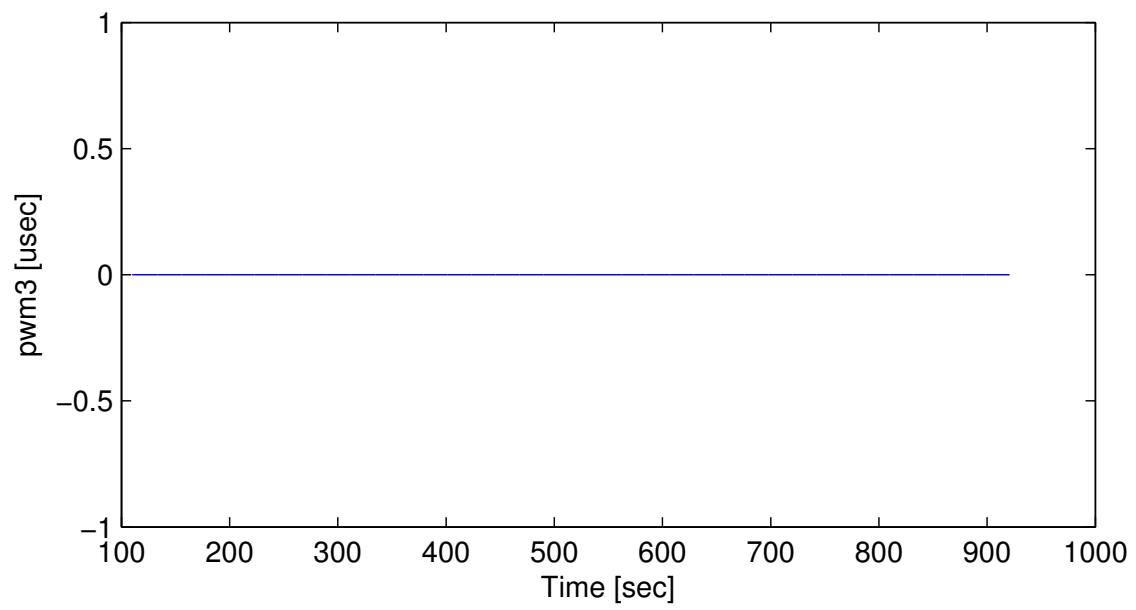


Figure E.60: pwm4 vs. Time

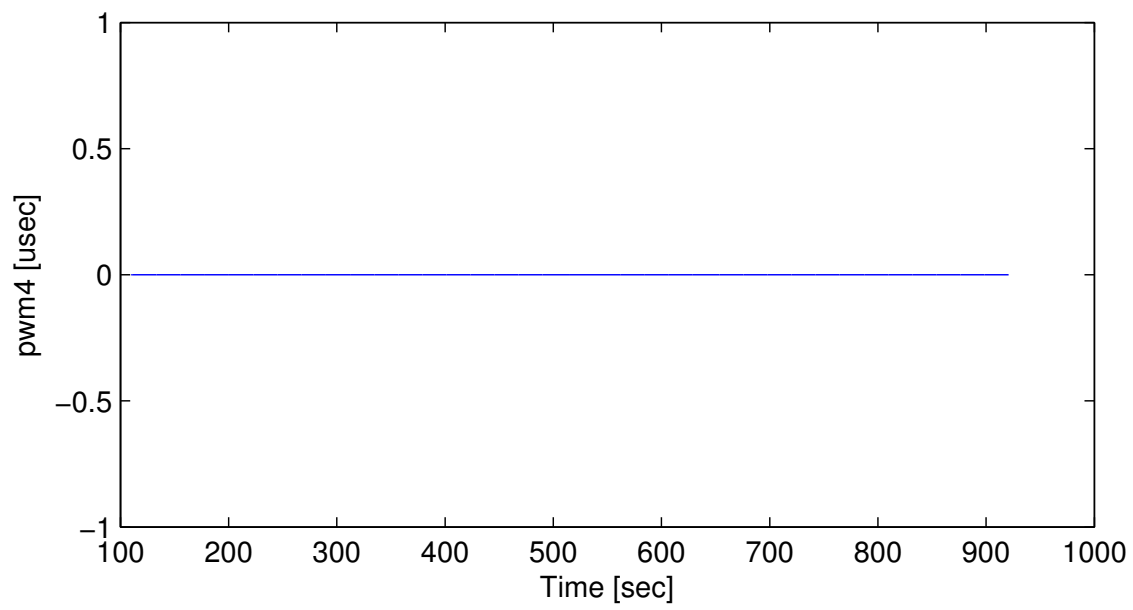

Figure E.61: pwm5 vs. Time

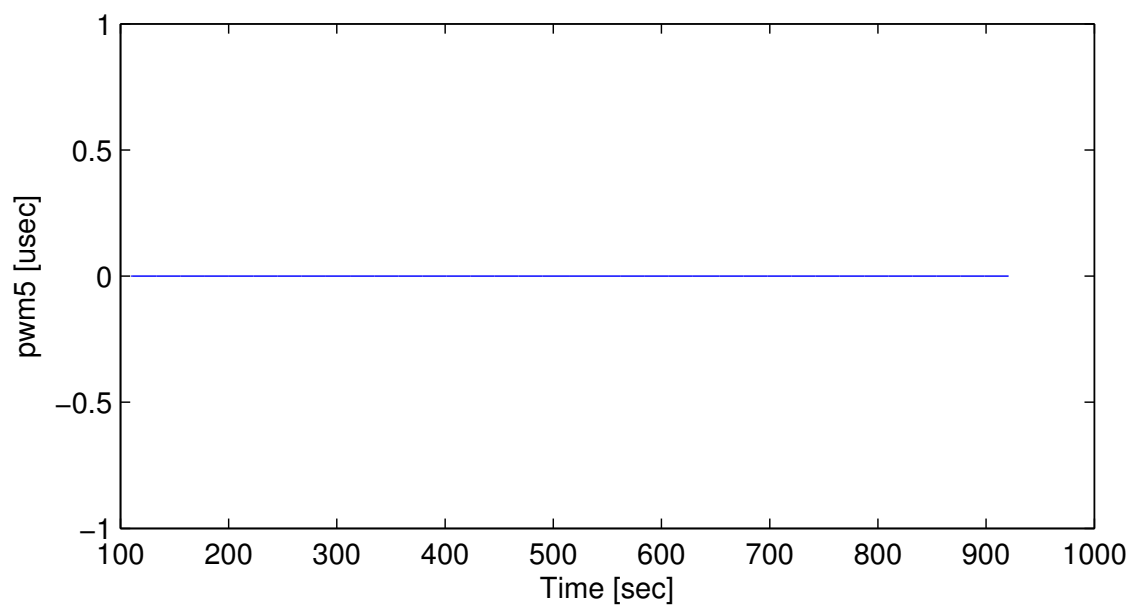

Figure E.62: pwm6 vs. Time

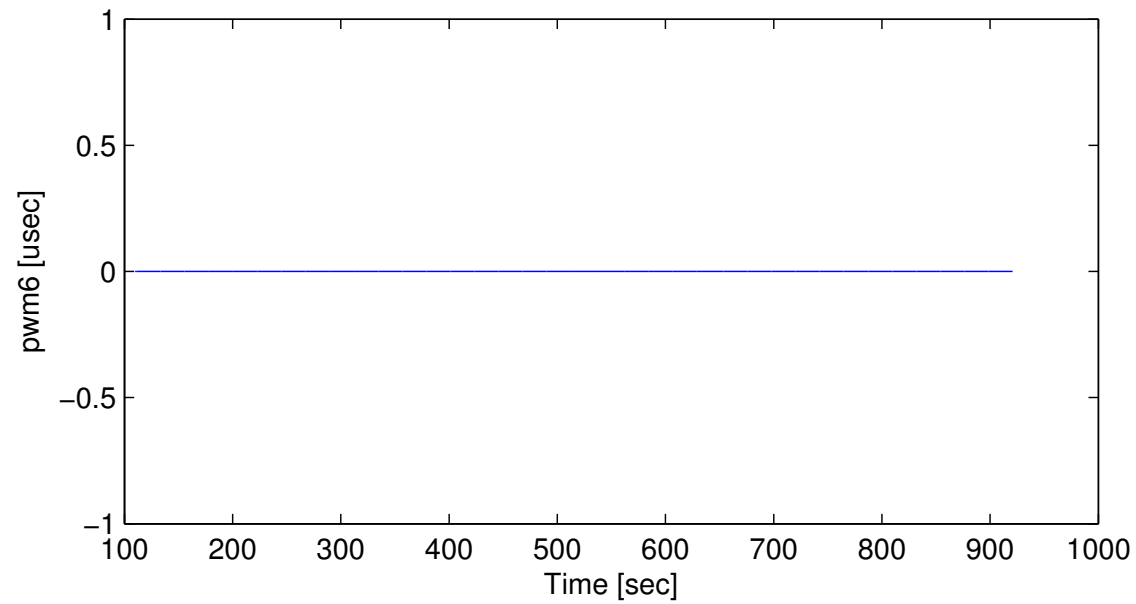


Figure E.63: pwm7 vs. Time

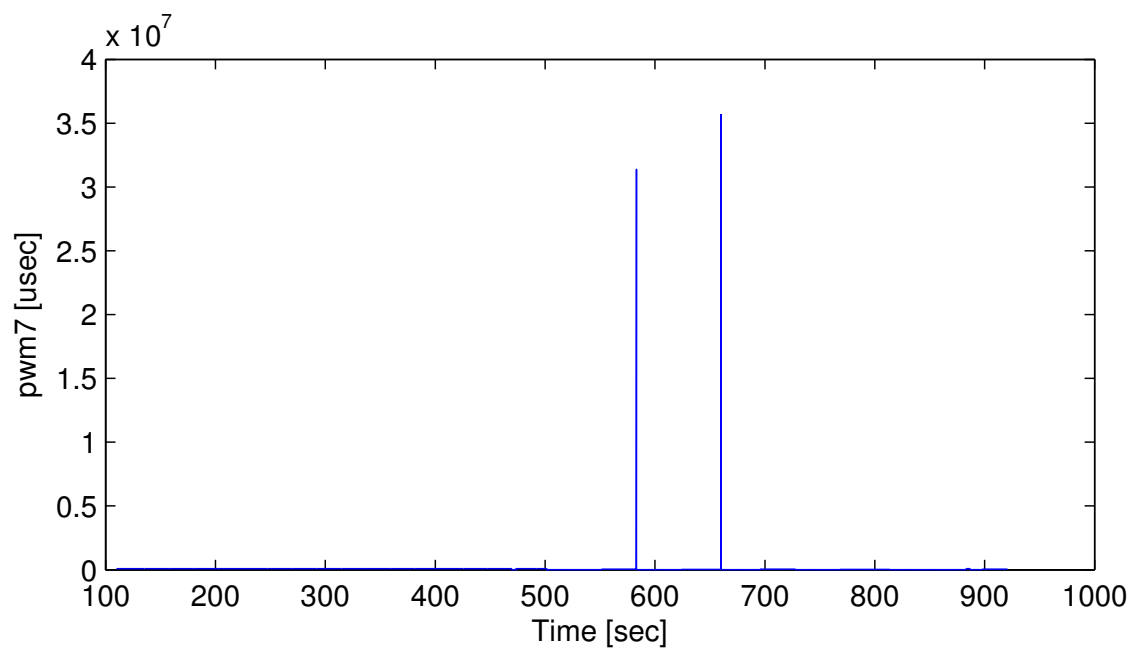

Figure E.64: deltaT vs. Time

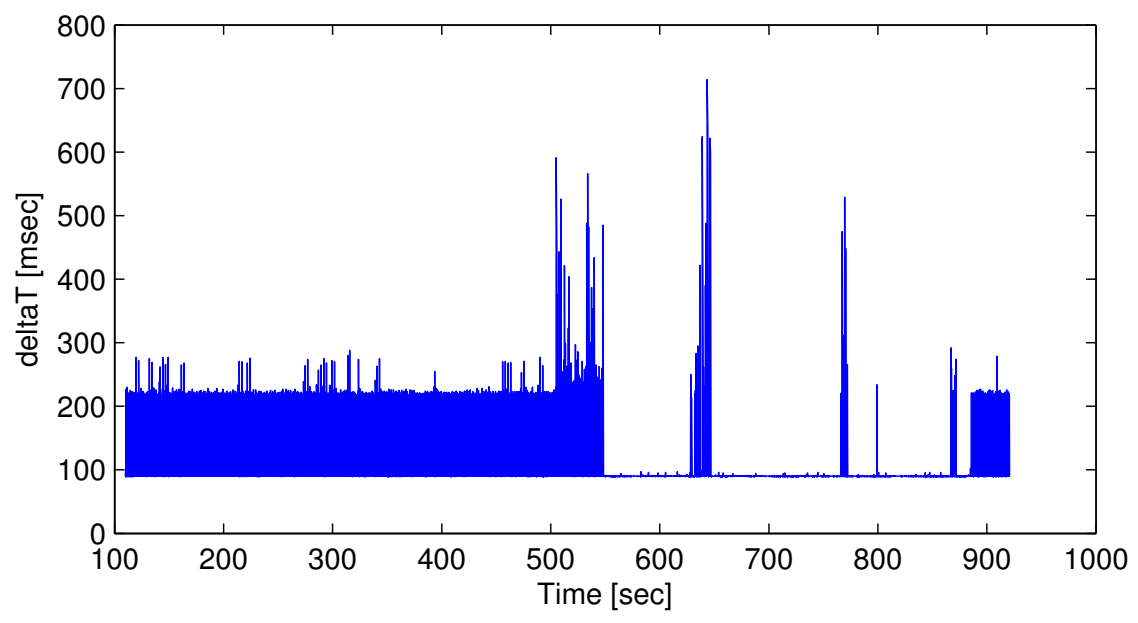




\section{E.3 Sample System Ouput - State Data}

Figure E.65: qbar vs. Time

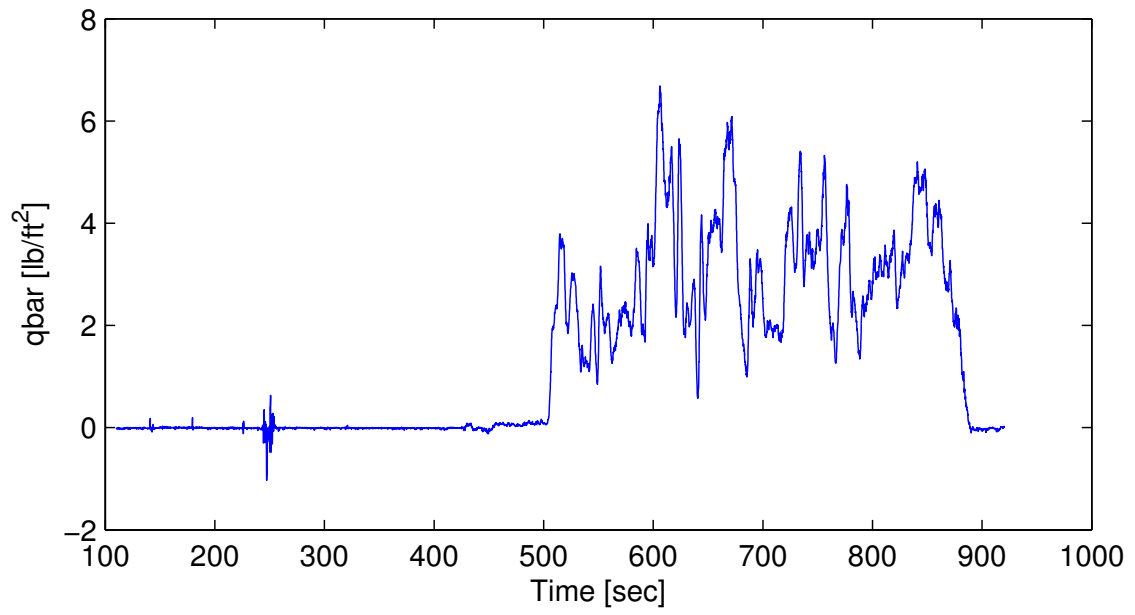

Figure E.66: rho vs. Time

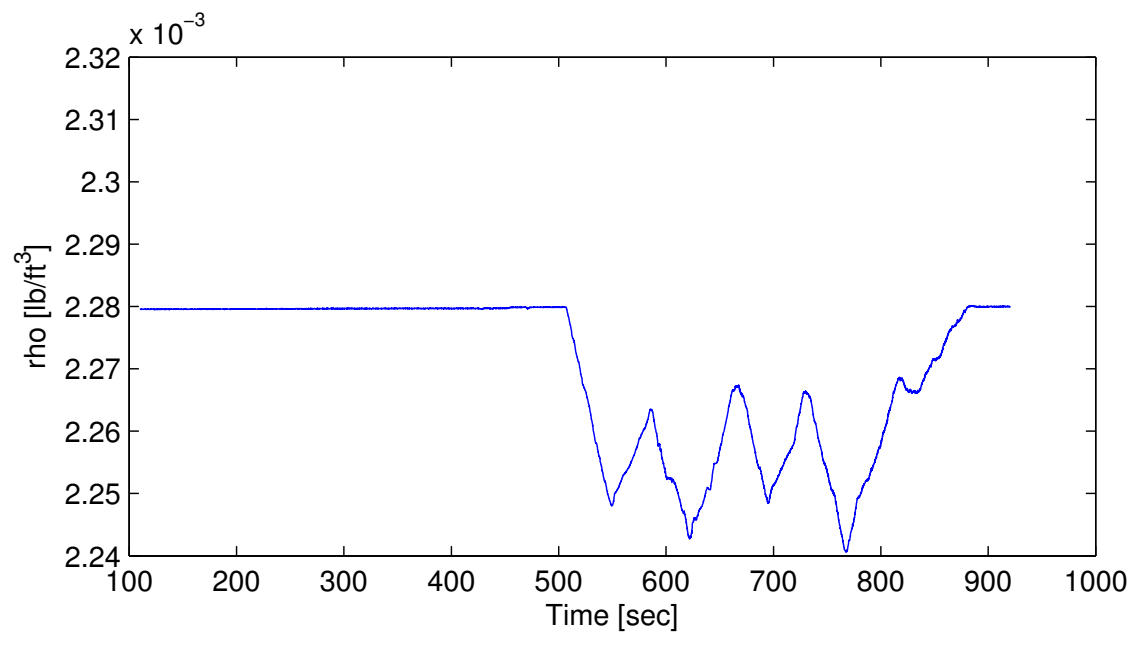


Figure E.67: vinf vs. Time

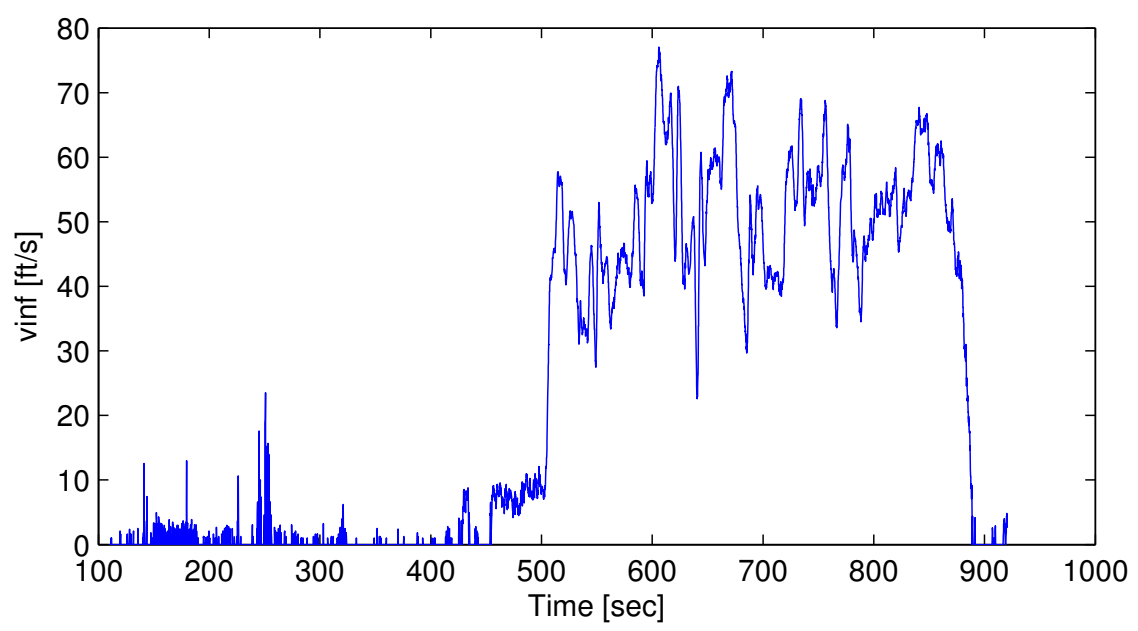

Figure E.68: alphaP vs. Time

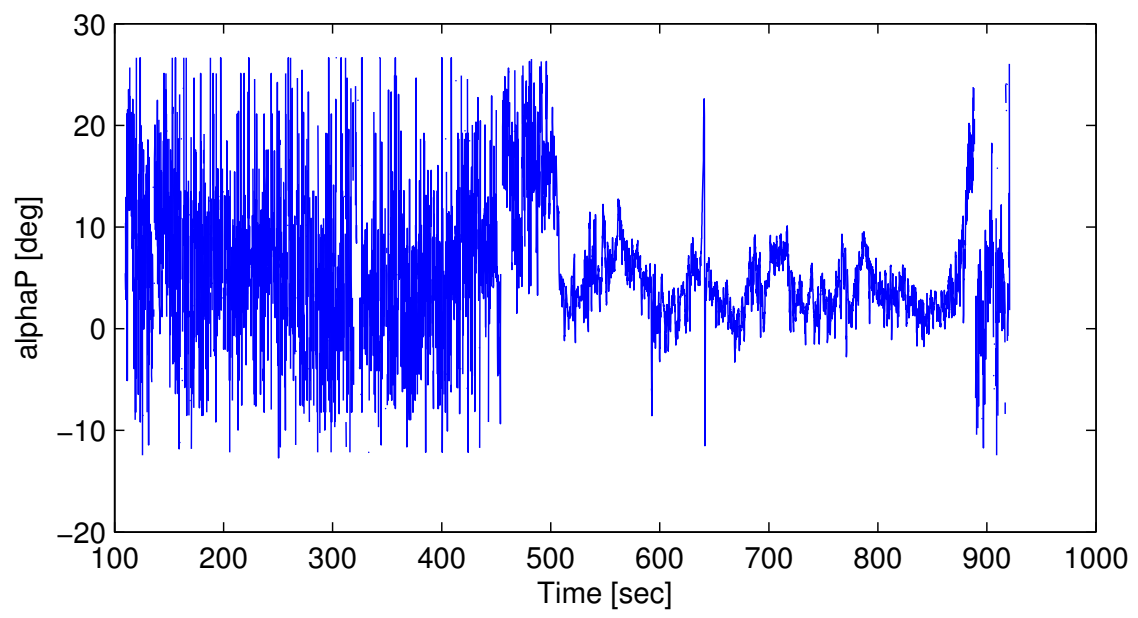

Figure E.69: betaP vs. Time

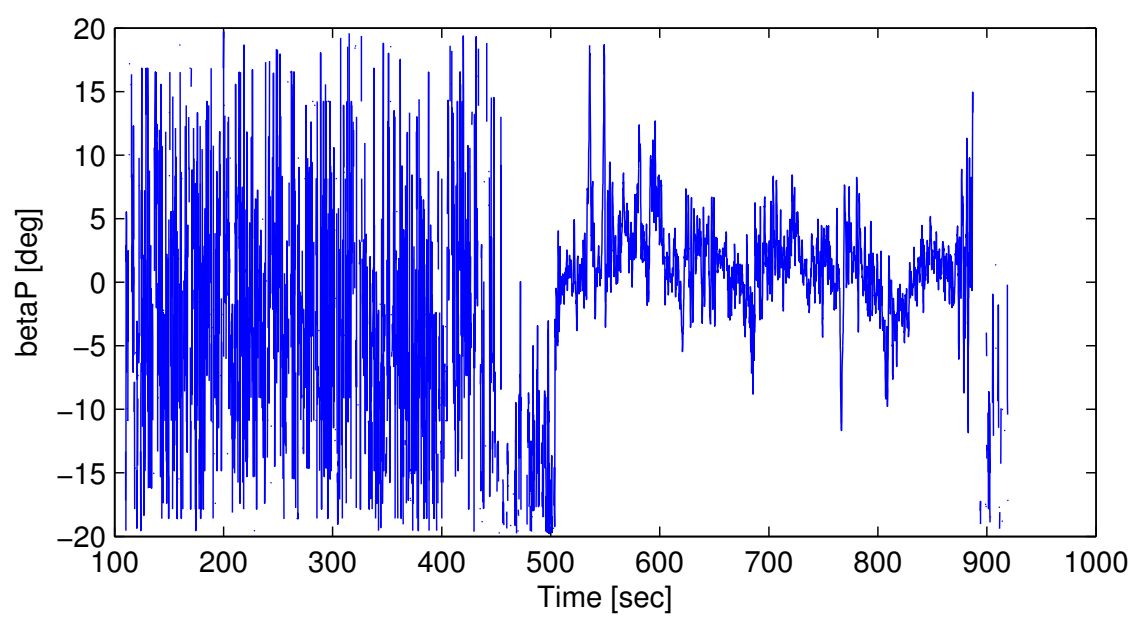


Figure E.70: rollRate vs. Time

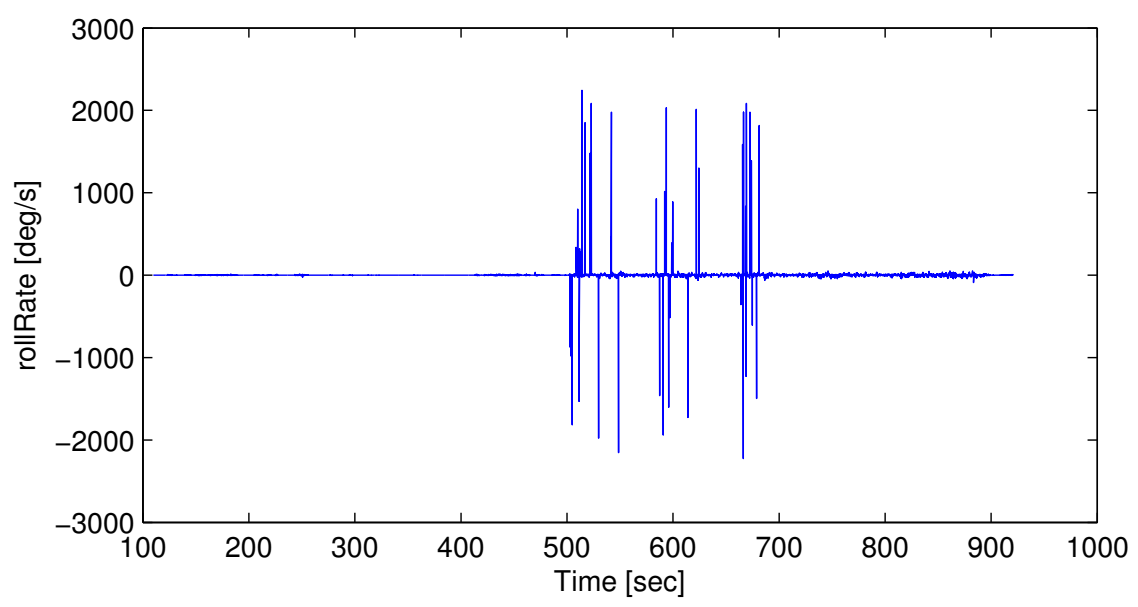

Figure E.71: pitchRate vs. Time

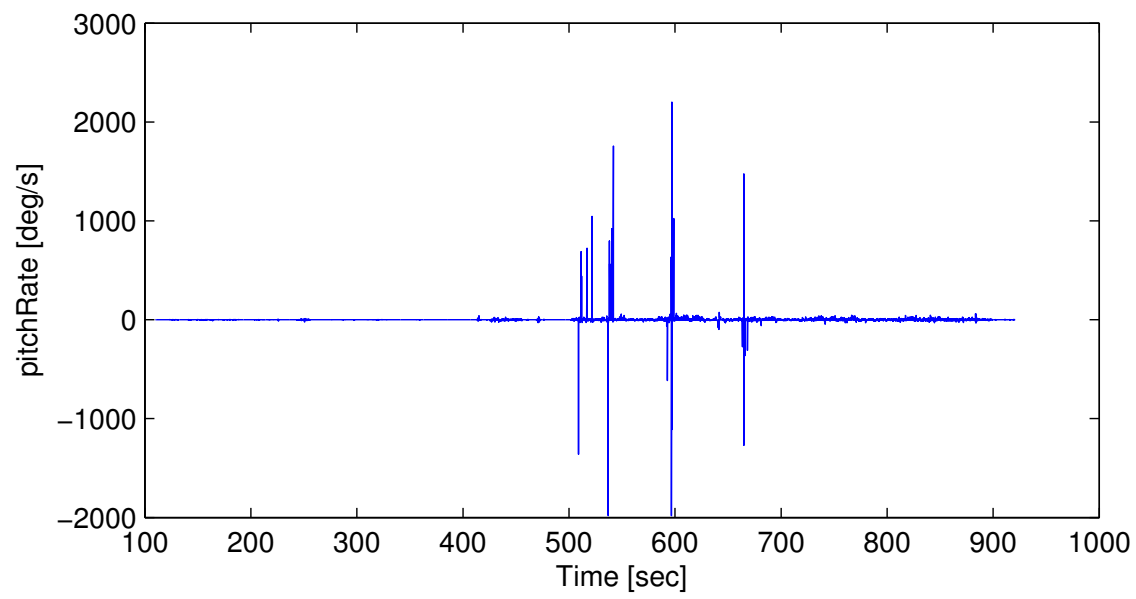

Figure E.72: yawRate vs. Time

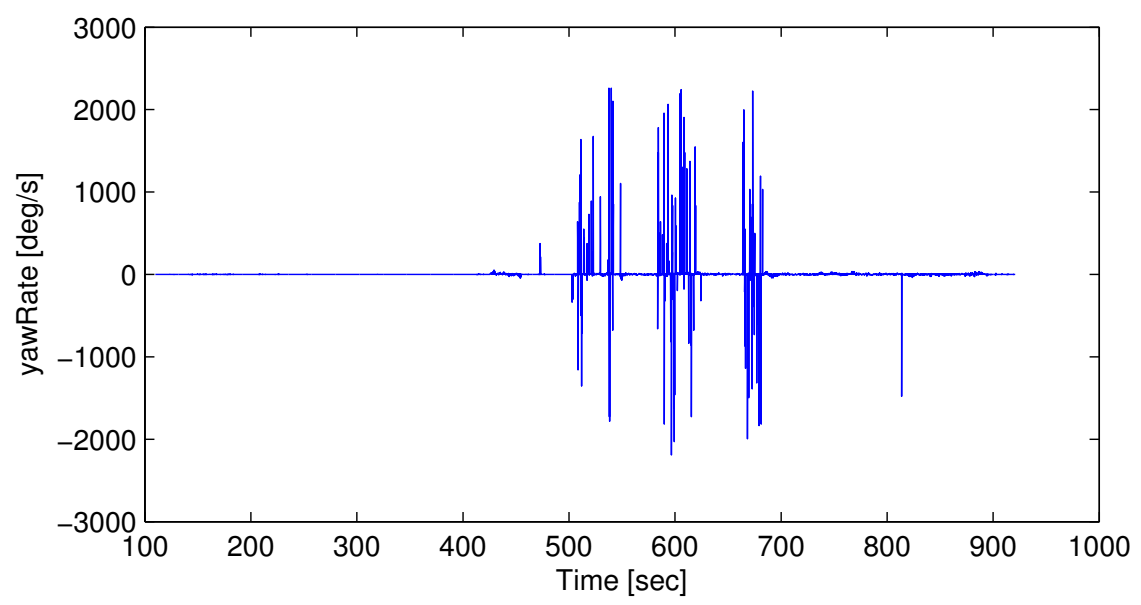


Figure E.73: accelX vs. Time

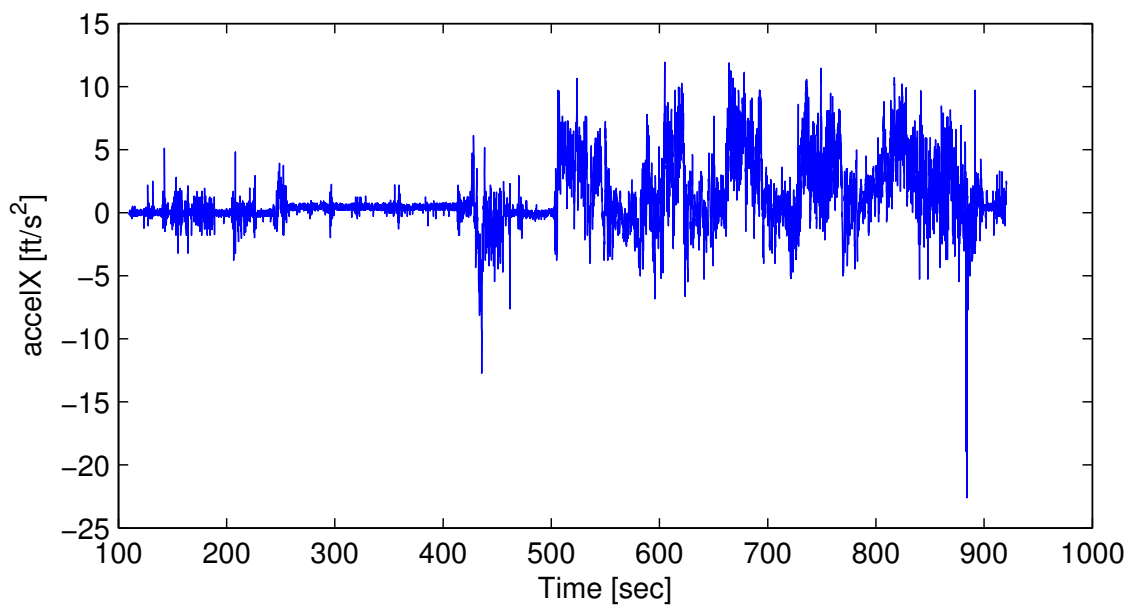

Figure E.74: accelY vs. Time

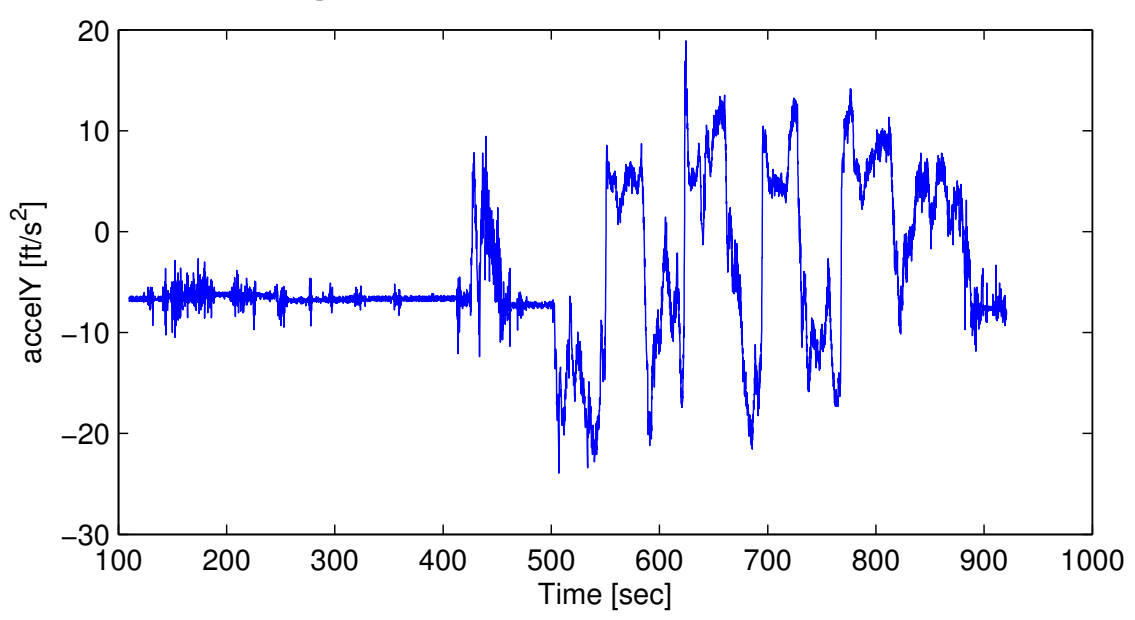

Figure E.75: accelZ vs. Time

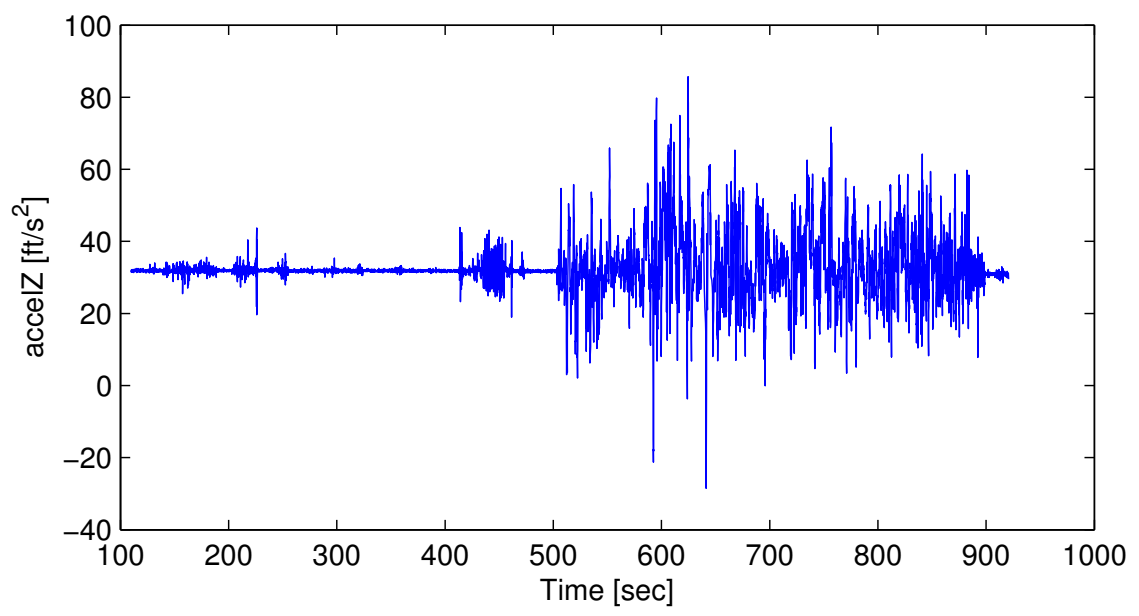


Figure E.76: CD vs. Time

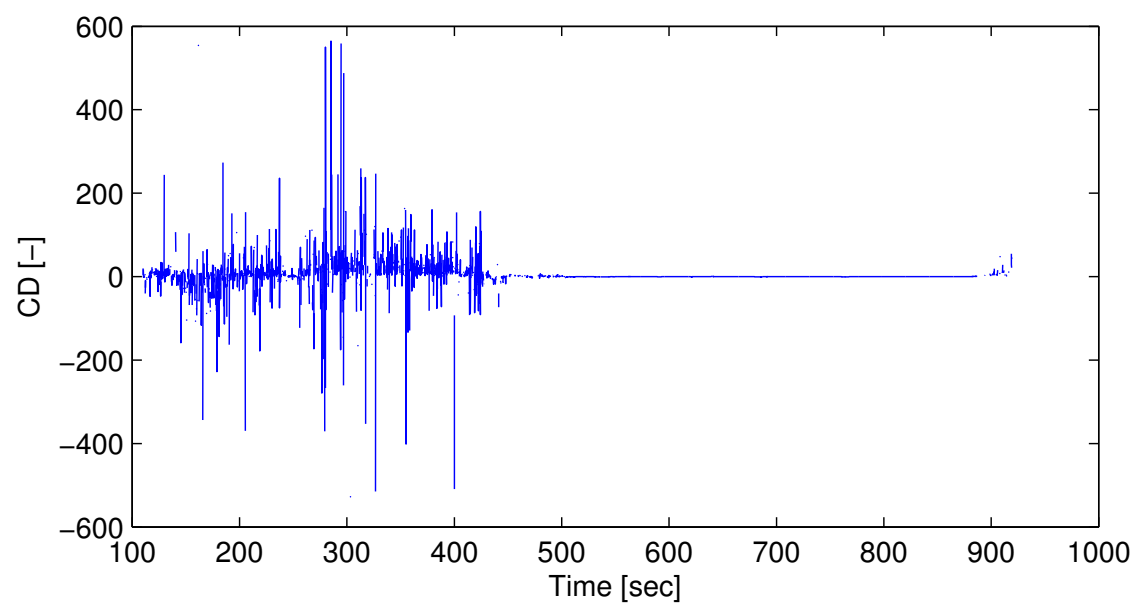

Figure E.77: CY vs. Time

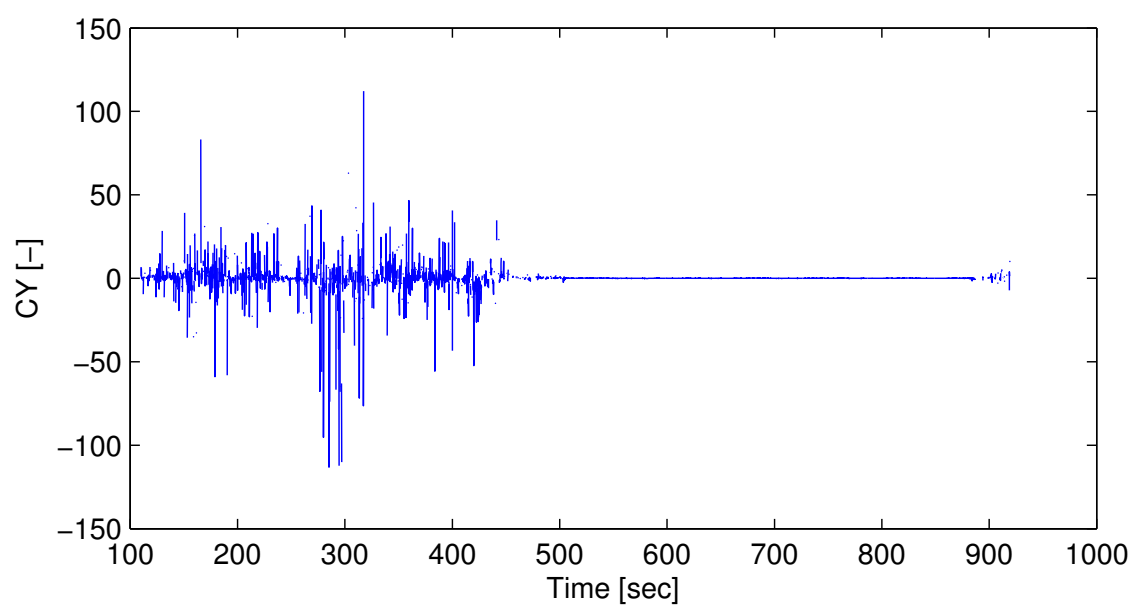

Figure E.78: CL vs. Time

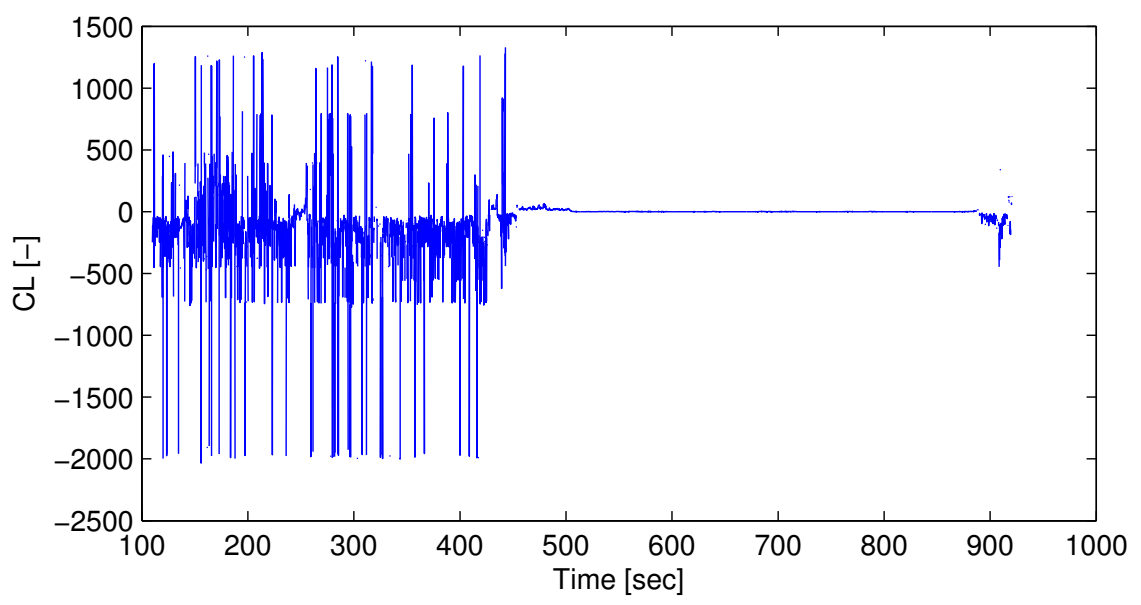


Figure E.79: D vs. Time

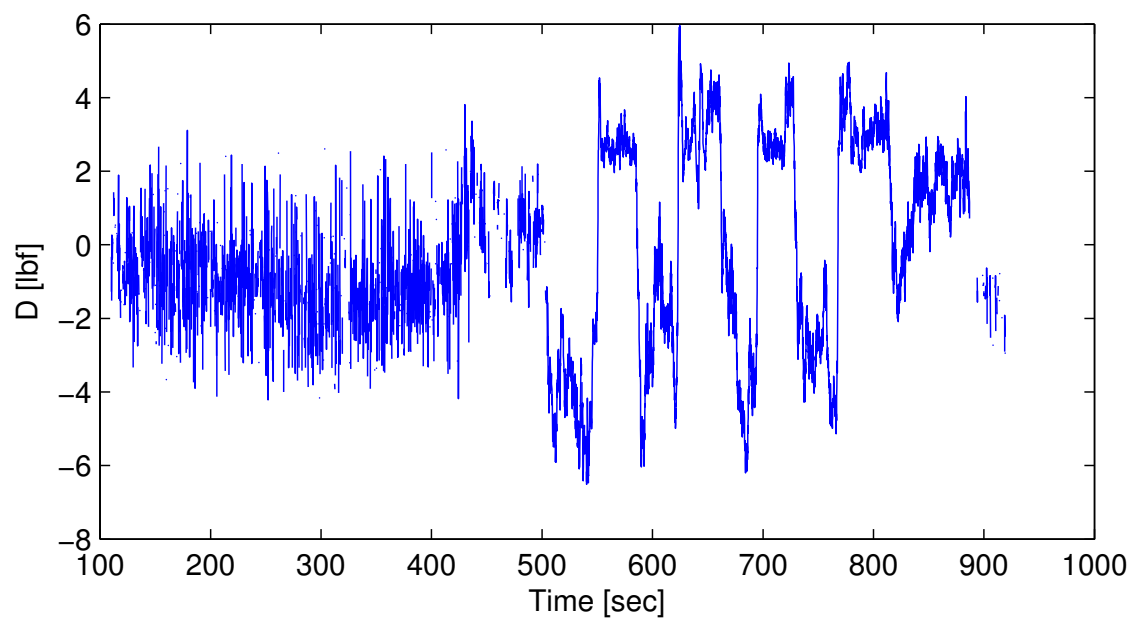

Figure E.80: Y vs. Time

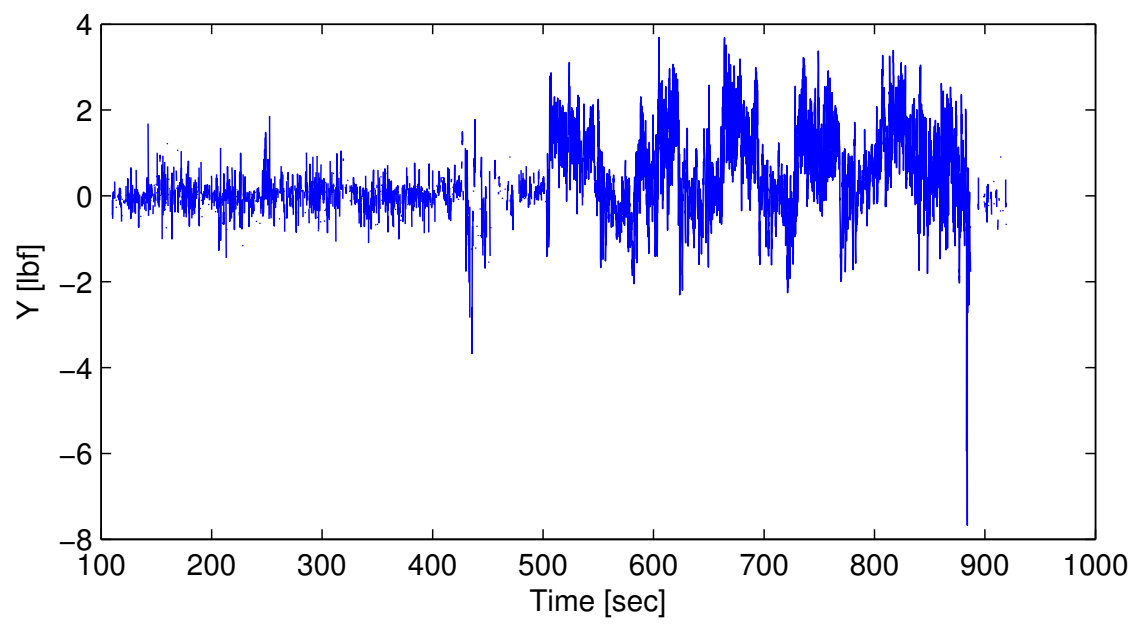

Figure E.81: L vs. Time

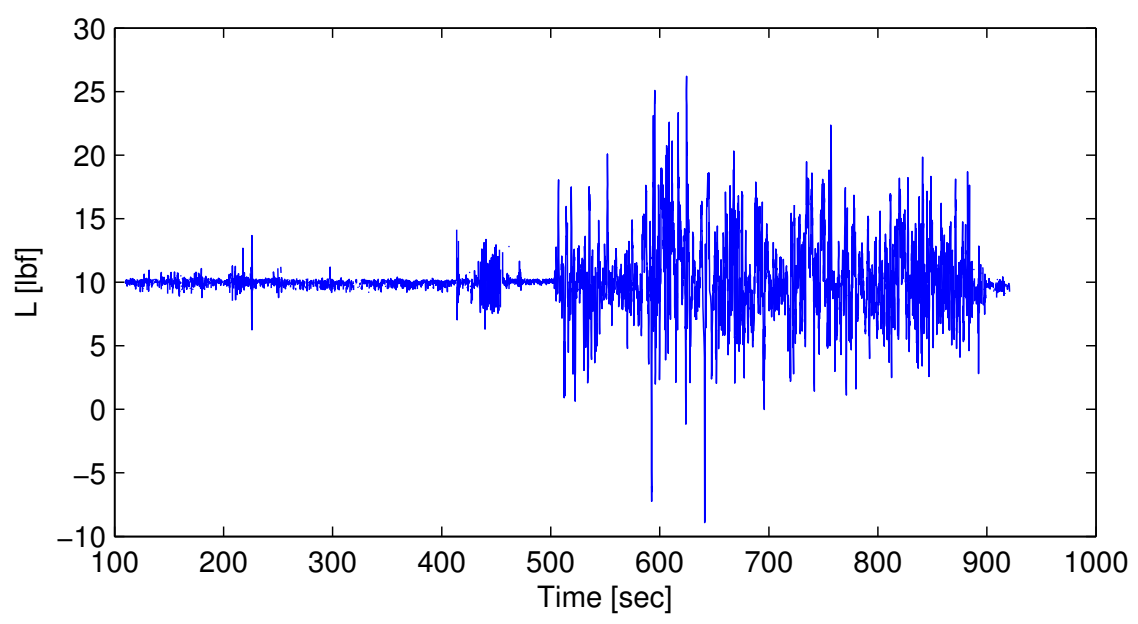

